\title{
THE EMBEDDINGS OF THE DISCRETE SERIES IN THE PRINCIPAL SERIES FOR SEMISIMPLE LIE GROUPS OF REAL RANK ONE \\ BY
}

\author{
M. WELLEDA BALDONI SILVA
}

\begin{abstract}
We consider the problem of finding all the "embeddings" of a discrete series representation in the principal series in the case of a simple real Lie group $G$ of real rank one. More precisely, we solve the problem when $G$ is $\operatorname{Spin}(2 n, 1)$, $\mathrm{SU}(n, 1), \operatorname{Sp}(n, 1)$ or $F_{4}(n>2)$.

The problem is reduced to considering only discrete series representations with trivial infinitesimal character, by means of tensoring with finite dimensional representations.

Various other techniques are employed.
\end{abstract}

1. Introduction. Let $G$ be a connected semisimple Lie group, with finite center. In two basic papers ([3], [4]), Harish-Chandra gave an explicit parametrization of the discrete series representations for such a group $G$ and a way, in principle, for computing the characters. In his work there is little, if any, indication of how the discrete series can be realized: W. Schmid (Ph.D thesis, University of California, Proc. Nat. Acad. Sci. U.S.A. 59 (1968), 56-59) proved, under suitable conditions, an explicit formula for the restriction of a discrete series of $G$ to a maximal compact subgroup $K$ (Blattner's conjecture). In a subsequent paper with $\mathrm{H}$. Hecht [6], he proved Blattner's conjecture under the further assumption that $G$ is a linear group. He also fully characterized discrete series representations by their restriction to $K$ (minimal $K$-type theorem). The minimal $K$-type theorem was proved almost simultaneously by $\mathrm{N}$. Wallach using the theory of Enright-Varadarajan modules ([19], [15]).

In this paper we use the existence of minimal $K$-types to find all the possible embeddings of a discrete series representation in a nonunitary principal series, if $G$ is $\operatorname{Spin}(2 n, 1)(n \geqslant 2), \operatorname{SU}(n, 1)(n \geqslant 2), \operatorname{Sp}(n, 1)(n \geqslant 2)$, or the connected Lie group $F_{4}$ with Lie algebra $f_{4(-20)}$.

Using G. Zuckerman's idea of tensoring with finite dimensional representations [22], the problem is first reduced to the study of the discrete series with trivial infinitesimal character. We also remark that if a discrete series is realized by a principal series, then the " $M$-type" of the principal series has to be in the minimal $K$-type of the discrete series.

Received by the editors October 20, 1978 and, in revised form, December 20, 1978.

AMS (MOS) subject classifications (1970). Primary $22 \mathrm{E} 45$.

Key words and phrases. Representations of semisimple Lie groups, real rank, branching theorems, minimal $K$-types, irreducible component. 
With this in mind, we compute the $M$-type of the minimal $K$-type, using the branching theorems from $K$ to $M$, set up by the author in [1]. The results obtained in this direction together with some special results concerning $F_{4}$, make it possible to prove

THEOREM 17.9. A discrete series representation is realized in a nonunitary principal series with multiplicity at most one.

This, together with a formula ([14], [21]) which relates the character of a discrete series to the character of a principal series, is used to prove that the number of occurrences of a discrete series as a subquotient, but not as a quotient, is odd. We solve completely the problem for the class of groups considered. In the case of $\operatorname{Sp}(n, 1)$ (resp. $\left.F_{4}\right)$, relative to a particular discrete series, we also use a result of [8] (resp. [25]), together with $K$-type considerations. The results for $\operatorname{Spin}(n, 1)$ and $\mathrm{SU}(n, 1)$ were already known (see for example [24], [26]).

Finally, we would like to point out that the main results of this paper can be found in the tables in $\S 18$. To obtain them, we also use explicit realizations of the discrete series by A. Knapp and N. Wallach [10].

Much of this paper was contained in the author's doctoral thesis [1]. The author wishes to thank her thesis advisor, Professor Nolan R. Wallach for his continuous encouragement and help.

2. Preliminaries. The following notations will be in force throughout the paper. We let $G$ be a connected semisimple Lie group with finite center, and fix a Cartan decomposition $\mathfrak{g}=\mathfrak{f} \oplus \mathfrak{p}$ of the Lie algebra.

Let $G_{\mathbf{C}}$ be a connected, simply connected complex Lie group with Lie algebra $g_{\mathbf{C}}$. We assume that $G$ is the real analytic subgroup of $G_{C}$ corresponding to $g$ and that rank $g=$ rank $f$.

Let $\mathfrak{t} \subset$ be a compact Cartan subalgebra for $g$. Let $\Delta$ be the set of roots of $\left(g_{C}, t_{C}\right)$ and let $\Delta_{k}$ and $\Delta_{n}$ be the sets of compact and noncompact roots, respectively. The Weyl groups of $\Delta$ and $\Delta_{k}$ are $W$ and $W_{K}$.

If $P$ is a system of positive roots for $\Delta$, we let $P_{k}$ and $P_{n}$ be the obvious sets of positive roots. In terms of $P$ we define

$$
\delta_{n}^{P}=\frac{1}{2} \sum_{\alpha \in P_{n}} \alpha, \quad \delta_{k}^{P}=\frac{1}{2} \sum_{\alpha \in P_{k}} \alpha \quad \text { and } \quad \delta_{P}=\delta_{k}^{P}+\delta_{n}^{P} .
$$

Let $K$ and $T$ be the analytic subgroups corresponding to $f$ and $t$.

We need also to introduce notation for an Iwasawa Cartan.

Let $a$ be a maximal abelian subalgebra of $\mathfrak{p}$. Form $\Lambda$, the set of restricted roots of $(g, a)$. Let $m$ be the centralizer of $a$ in $\mathfrak{t}^{2}$ Let $\mathfrak{h}^{-} \subset \mathfrak{t}$ be a Cartan subalgebra of $m$ and $\mathfrak{h}=\mathfrak{h}^{-} \oplus \mathfrak{a}$. Then $\mathfrak{h}$ is a Cartan subalgebra of $\mathfrak{g}$.

Let $\Phi$ denote the root system of $\left(g_{C}, \mathfrak{h}_{C}\right)$ and $\Phi_{m} \subset \Phi$ be the root system of $\left(\mathfrak{m}_{\mathbf{C}}, \mathfrak{h}_{\mathbf{C}}^{-}\right)$.

Let $\Phi^{+}$be a positive root for $\Phi$ obtained by requiring that a comes before $\mathfrak{h}^{-}$. Form $\Lambda^{+}$the corresponding restricted positive root and let $\Phi_{m}^{+}=\Phi_{m} \cap \Phi^{+}$. Let $\mathfrak{n}$ be the sum of the positive restricted root spaces. Then $\mathfrak{g}=\mathfrak{f} \oplus \mathfrak{a} \oplus \mathfrak{n}$ is an Iwasawa decomposition of $\mathfrak{g}$. 
Later we shall use the notation $A$ and $N$ for the analytic subgroups with Lie algebras $a$ and $\mathfrak{n}, M$ for the centralizer of $a$ in $K$, and $\rho$ for half the sum of the positive restricted roots, counted with multiplicities. We also set $\delta_{m}=\frac{1}{2} \Sigma_{\alpha \in \Phi_{m}^{+}} \alpha$. Let $($,$) denote the dual of the Killing form B$ of $g$ restricted to $\mathfrak{h}$ or $\mathrm{t}$.

3. Harish-Chandra homomorphism. If $\tilde{\mathfrak{h}}$ is a Cartan subalgebra of $\mathfrak{g}$, let $U(\mathfrak{g})$ and $U(\tilde{\mathfrak{h}})$ denote the universal enveloping algebra of $\tilde{\mathfrak{g}}_{\mathbf{C}}$ and $\tilde{\mathfrak{h}}_{\mathbf{C}}$ respectively. Let $z$ be the center of $U(\mathfrak{g})$. Then there exists a homomorphism $\gamma_{\tilde{\mathfrak{h}}}$ of $z$ onto $(U(\tilde{\mathfrak{h}}))^{W}$, the Weyl group invariants of $U(\tilde{\mathfrak{h}}) . \gamma_{\tilde{\mathfrak{h}}}$ is called the Harish-Chandra homomorphism. If $\lambda \in \tilde{\mathfrak{h}}_{\mathbf{C}}$, we denote by $\chi_{\lambda}: \mathfrak{z} \rightarrow \mathbf{C}$ the homomorphism defined by $\chi_{\lambda}(Z)=\lambda\left(\gamma_{\mathfrak{h}}(Z)\right)$. $\chi_{\lambda}$ is the infinitesimal character of the Verma module of highest weight $\lambda-\delta_{\Psi^{+}}$, where $\delta_{\Psi^{+}}=\frac{1}{2} \sum_{\alpha \in \Psi^{+}} \alpha$ relative to some choice, $\Psi^{+}$, of positive roots for the root $\Psi$ of $\left(g_{\mathbf{C}}, \tilde{\mathfrak{h}}_{\mathbf{C}}\right)$.

We recall the following properties:

(1) $\chi_{\lambda}=\chi_{\mu} \Leftrightarrow \lambda=\sigma \mu, \sigma$ in the Weyl group of $\Psi$.

(2) If $\mathfrak{h}^{\prime}$ is another Cartan of $\mathfrak{g}$ and $\operatorname{Ad}(x): \tilde{\mathfrak{h}}_{\mathbf{C}} \rightarrow \mathfrak{h}_{\mathbf{C}}^{\prime}$ for some $x \in G_{\mathbf{C}}$, then $\gamma_{\mathfrak{h}^{\prime}}=\operatorname{Ad}(x) \gamma_{\mathfrak{h}^{\prime}}$.

(3) If $\chi: z \rightarrow \mathbf{C}$ is a homomorphism, then there exist $\lambda \in \tilde{\mathfrak{h}}_{\mathbf{C}}$ such that $\chi=\chi_{\lambda}$.

4. Discrete series and principal series. Let $\hat{M}$ be the set of all equivalences classes of irreducible finite dimensional representations of $M$.

If $\xi \in \hat{M}$ we fix an element $\left(\xi, H^{\xi}\right)$ of $\xi$. For $\xi \in \hat{M}$ and $\nu \in a_{C}^{*}$ we define the principal series $\left(\pi_{\xi, \nu}, H^{\xi, \nu}\right)$ as follows:

(1) $H^{\xi, \nu}$ is the space of all measurable functions, $f: G \rightarrow H^{\xi}$ so that:

(i) $f($ gman $)=e^{-(\nu+\rho) l g a} \xi(m)^{-1} f(g)(1 g: A \rightarrow a$ is the inverse to exp: $a \rightarrow A)$.

(ii) $\int_{K}\|f(k)\|^{2} d k<\infty$.

(2) $\left(\pi_{\xi, \nu}(g) f\right)(x)=f\left(g^{-1} x\right)$ for $x, g \in G$.

Let $\Theta_{\xi, \nu}$ be the character of $\left(\pi_{\xi, \nu}, H^{\xi, \nu}\right)$. Let $\Lambda_{\xi}$ be the highest weight of $\xi$ relative to $\Phi_{m}^{+}$. For example, according to [5, p. 87], the infinitesimal character of the nonunitary principal series $\pi_{\xi, \nu}$ is $\chi_{\Lambda(\xi, \nu)}$, where $\Lambda(\xi, \nu) \in\left(\mathfrak{h}^{-} \oplus \mathfrak{a}\right)_{\mathbf{C}}$ is defined by $\Lambda(\xi, \nu)=\Lambda_{\xi}+\delta_{m}+\nu$.

Denote by $L_{T}$ the integral forms on $t_{C}$. The elements of $L_{T}$ are precisely those that lift to a character of $T$. We say that $\Lambda \in \mathfrak{h}^{*}$ is regular if $(\Lambda, \alpha) \neq 0$ for $\alpha \in \Delta$. Corresponding to each regular $\Lambda \in L_{T}$, Harish-Chandra has constructed certain invariant eigendistributions $\Theta_{\Lambda}$ on $G$ [3]. Two of these coincide precisely when their parameters $\Lambda$ are related by an element of the Weyl group of $K$. Each $\Theta_{\Lambda}$ is the character of a discrete series representation and conversely [4].

We denote by $\left(\pi_{\Lambda}, H^{\Lambda}\right)$ the discrete series with character $\Theta_{\Lambda}$. The infinitesimal character of $\pi_{\Lambda}$ is $\chi_{\Lambda}$.

5. Minimal $K$-type theorem. Let $(\pi, H)$ be a representation of $G$ on the Hilbert space $(H,\langle\rangle$,$) . We say that (\pi, H)$ is $K$-finite if

(1) $\left(\pi_{\mid K}, K\right)$ is unitary,

(2) a $K$-representation $H=\Sigma_{\gamma \in \hat{K}} H_{\gamma}$ (unitary direct sum) where $H_{\gamma}=m_{\gamma} V_{\gamma}$, $\left(\pi_{\gamma}, V_{\gamma}\right) \in \gamma \in \hat{K}$ and $m_{\gamma}<\infty$. 
Let $H_{F}$ denote the set of the $K$-finite vectors and let $\left(\pi, H_{F}\right)$ be the corresponding $g$ module.

If a discrete series representation is infinitesimally equivalent to an irreducible component of a (nonunitary) principal series we say, simply, that it is an irreducible component.

Let $\Lambda \in L_{T}$ be regular and let $\left(\tau_{\lambda}, V^{\lambda}\right)$ be a $K$-type of $\pi_{\Lambda}$.

The following result is an immediate consequence of the Frobenius reciprocity theorem.

THEOREM 5.1. If $\pi_{\Lambda}$ is an irreducible component of $\left(\pi_{\xi, \nu}, H^{\xi, \nu}\right)$, then $\xi$ is an $M$-type of $\tau_{\left.\lambda\right|_{M}}$.

Proof. By assumption, there exist $V_{2} \subset V_{1}$, closed, $\pi_{\xi, \nu}$ invariant subspace of $H^{\xi, \nu}$, such that $\left(\pi_{\xi, \nu},\left(V_{1} / V_{2}\right)_{F}\right)$ is algebraically equivalent to $\left(\pi_{\Lambda}, H_{F}^{\Lambda}\right)$.

By the compactness of $K$, we have that $\left(\pi_{\xi, \nu}, V_{1} / V_{2}\right)$ is equivalent as a $K$-representation to $\left(\pi_{\xi,\left.\nu\right|_{\kappa}}, W\right)$ where $W$ is a closed invariant subspace of $H^{\xi, \nu} . V_{1} / V_{2}$ is infinitesimally equivalent to $H^{\Lambda}$, forcing $W$ to be infinitesimally equivalent to $H^{\Lambda}$ as a $K$-module. Hence $\tau_{\lambda}$ is a $K$-type of $W$.

Since $\pi_{\xi, \nu}$, as a $K$-representation, is equivalent to the unitarily induced representation from $\xi$ to $K$, the theorem follows from the Frobenius reciprocity theorem.

\section{Conjugate dual representation.}

LEMMA 6.1. There is a nondegenerate (continuous) sesquilinear pairing ( , ) between $H^{\xi, \nu}$ and $H^{\xi,-\bar{\nu}}$ for each $\xi \in \hat{M}$ and $\nu \in \mathfrak{a}_{\mathbf{C}}^{*}$, so that if $f_{1} \in H^{\xi, \nu}$ and $f_{2} \in H^{\xi,-\bar{\nu}}$ then

$$
\left(\pi_{\xi, \nu}(g) f_{1}, \pi_{\xi,-\bar{\nu}}(g) f_{2}\right)=\left(f_{1}, f_{2}\right) \quad(g \in G) .
$$

Proof. See e.g. [18, Lemma 3.1].

Definition 6.2. Let $(\pi, H)$ be a representation of $G$ on a Hilbert space $H$. We denote by $H^{*}=\{f: H \rightarrow \mathbf{C}, f$ continuous and linear, such that $f(\alpha x)=\bar{\alpha} f(x)$, for $\alpha \in \mathbf{C}$ and $x \in H\}$. The conjugate dual representation $\left(\pi^{*}, H^{*}\right)$ of $(\pi, H)$ is defined by

$$
\pi^{*}(g) \lambda=\lambda \circ \pi\left(g^{-1}\right) \text { for } \lambda \in H^{*} \text { and } g \in G .
$$

LeMma 6.3. Let $\pi$ be a $K$-finite representation on $(H,()$,$) . Assume that \pi$ is unitarizable, i.e., there exist $\langle$,$\rangle an inner product on H_{F}$, so that $\langle\pi(X) v, w\rangle=$ $-\langle v, \pi(X) w\rangle$, for $X \in \mathfrak{g}$ and $v, w \in H_{F}$, and that $\left(\pi, H_{F}\right)$ is irreducible. Then $\pi^{*}$ is infinitesimally equivalent to $\pi$.

Proof. If $f \in\left(H^{*}\right)_{F}$, then there exist $S \subseteq \hat{K}$ so that $f \circ E_{S}=f$ ( $E_{S}$ is the projection of $H$, determined by $S$ ).

Let $V$ be the completion of $H_{F}$ relative to $\langle$,$\rangle . Then, since E_{S} V \subset H_{F}$ and $\operatorname{dim} E_{S} V<\infty$, we see that $f$ extends to a continuous antilinear functional on $V$. Let $A(f) \in V$ be defined by $f(v)=\langle\overline{v, A(f)}\rangle$. Now $A(f) \perp\left(E_{S} V\right)^{\perp}$ ( $\perp$ is relative to $\langle$,$\rangle ), thus A(f) \in E_{S} V \subset H_{F}$. We therefore have $A:\left(H^{*}\right)_{F} \rightarrow H_{F}$ a linear operator. Also if $A(f)=0$, then $f=0$. Now

$$
\left\langle\overline{v, A \pi^{*}(X) f}\right\rangle=\left(\pi^{*}(X) f\right) v=-f \pi(X) v=-\langle\overline{\pi(X) v, A f}\rangle=\langle\overline{v, \pi(X) A f}\rangle .
$$


Thus $A \pi^{*}(X) f=\pi(X) A f$.

Hence $A:\left(\pi^{*},\left(H^{*}\right)_{F}\right) \rightarrow\left(\pi, H_{F}\right)$ is an injective intertwining operator. Since $\left(\pi, H_{F}\right)$ is irreducible, $A$ is also surjective.

Let $\Lambda \in L_{T}$ be regular and let $\xi \in \hat{M}, \nu \in a_{C}^{*}$.

THEOREM 6.4. $\pi_{\Lambda}$ is an irreducible component of $\pi_{\xi, \nu}$ iff $\pi_{\Lambda}$ is an irreducible component of $\pi_{\xi,-\bar{\nu}}$. More precisely:

(1) $\pi_{\Lambda}$ is a subspace (quotient) of $\pi_{\xi, \nu}$ iff $\pi_{\Lambda}$ is a quotient (subspace) of $\pi_{\xi,-\bar{\nu}}$.

Proof. Let ( , ) be the sesquilinear form of Lemma 6.1. Let $A: H^{\xi,-\bar{\nu}} \rightarrow\left(H^{\xi, \nu}\right)^{*}$ be defined by

$$
f_{2} \leadsto \overline{\left(\cdot, f_{2}\right)} \text { for } f_{2} \in H^{\xi,-\bar{\nu}} .
$$

Then $\left(A \pi_{\xi,-\bar{\nu}}(g) f_{2}\right) f_{1}=\left(\pi_{\xi, \nu}^{*}(g) A f_{2}\right) f_{1}$ for $g \in G, f_{1} \in H^{\xi, \nu}$ and $f_{2} \in H^{\xi,-\bar{\nu}}$.

Since $A$ is a continuous bijection of $H^{\xi,-\bar{\nu}}$ onto $H^{\xi, \nu}$, then $\pi_{\xi,-\bar{\nu}}$ is $G$-equivalent to $\pi_{\xi, \nu}^{*}$.

If $V \subset H^{\xi, \nu}$ is a closed invariant subspace, define $V^{\perp}=\left\{x \in H^{\xi,-\bar{\nu}}\right.$ such that $(v, x)=0$ for all $v \in V\}$. Then $V^{\perp}$ is a closed invariant subspace of $H^{\xi,-\bar{\nu}}$. Now let $V_{2} \subset V_{1} \subset H^{\xi, \nu}$ be closed and invariant subspaces.

Assume that $V_{1} / V_{2}$ is infinitesimally equivalent to $H^{\Lambda}$. Define $\phi: V_{1} / V_{2} \times$ $V_{2}^{\perp} / V_{1}^{\perp} \rightarrow \mathrm{C}$ by $\phi(\bar{v}, \bar{w})=(v, w)$, where $\bar{v} \in V_{1} / V_{2}$ and $\bar{w} \in V_{2}^{\perp} / V_{1}^{\perp}$. It is immediate to check that $\phi$ is indeed well defined and it is a nondegenerate continuous (relative to the obvious topology) sesquilinear form. Moreover $\phi\left(\pi_{\xi, \nu}(g) \bar{v}, \pi_{\xi,-\bar{\nu}}(g) \bar{w}\right)=\phi(\bar{v}, \bar{w})$. Hence

$$
\left(\pi_{\xi, \nu}^{*},\left(V_{1} / V_{2}\right)^{*}\right) \stackrel{G \text { equivalent }}{\simeq}\left(\pi_{\xi,-\bar{\nu}}, V_{2}^{\perp} / V_{1}^{\perp}\right) .
$$

Since $H_{F}^{\Lambda}$ is equivalent to $\left(V_{1} / V_{2}\right)_{F}$, Lemma 6.3 says that $\left(V_{1} / V_{2}\right)_{F}$ is equivalent to $\left(V_{1} / V_{2}\right)_{F}^{*}$. Now apply (i) to get that $\pi_{\Lambda}$ is infinitesimally equivalent to a subquotient of $\pi_{\xi,-\bar{\nu}}$. By duality the first assertion of the theorem is proved and (1) follows immediately. We need the following results due to Langlands [12]. If $\lambda \in \Lambda^{+}$let $h_{\lambda} \in a$ be defined by $B\left(H, h_{\lambda}\right)=\lambda(H)$ for $H \in a$. We write $\operatorname{Re} \nu>0(<0)$ if $(\operatorname{Re} \nu, \lambda)=\left(\operatorname{Re} \nu, h_{\lambda}\right)>0(<0)$ for $\lambda \in \Lambda^{+}$.

THEOREM 6.5. Let $\xi \in \hat{M}$ and $\nu \in a_{C}^{*}$ such that $\operatorname{Re} \nu>0$. Then $\left(\pi_{\xi, \nu}, H_{F}^{\xi, \nu}\right)$ has $a$ unique irreducible quotient, which is not a discrete series.

Corollary 6.6. If $\nu \in a_{C}^{*}$ and $\operatorname{Re} \nu<0$, then $\left(\pi_{\xi, \nu}, H_{F}^{\xi, \nu}\right)$ has a unique irreducible subspace, which is not a discrete series.

7. A necessary condition for the infinitesimal character. We now make the further assumption that $\operatorname{dim} a=1$. For the moment we fix $P$, a positive root system for the roots $\Delta$ of $\left(\mathrm{g}_{\mathbf{C}}, \mathrm{t}_{\mathbf{C}}\right)$. Let $\delta=\delta_{P}$ and let $\pi_{\delta}$ be the corresponding discrete series. Let $\Phi^{+}, \Phi_{m}^{+}$be as in $\S 2$ and let $\alpha$ be the unique real positive root determined by $\Phi^{+}$.

LEMMA 7.1. If $\pi_{\delta}$ is an irreducible component of $\pi_{\xi, \nu}$ then $\nu \in a^{*}$ and $\nu \neq 0$. More precisely, if $\pi_{\delta}$ is a subspace of $\pi_{\xi, \nu}$, then:

(1) $\Lambda(\xi, \nu)=\delta_{Q}$ where $Q$ is positive for $\Phi$ and contains $\Phi_{m}^{+}$and $\alpha$; in particular $\nu\left(h_{\alpha}\right)>0$. 
Proof. If $\pi_{\delta}$ is an irreducible component of $\pi_{\xi, \nu}$, then $\chi_{\Lambda(\xi, \nu)}=\chi_{\delta_{\Phi^{+}}}$. Hence $\Lambda(\xi, \nu)=\Lambda_{\xi}+\delta_{m}+\nu=\sigma\left(\delta_{\Phi^{+}}\right)$for some $\sigma \in W(\Phi)$. But $\sigma\left(\delta_{\Phi^{+}}\right)\left(h_{\alpha}\right)=\nu\left(h_{\alpha}\right)$ and hence $\nu$ is real and different from zero, as asserted.

To prove (1) just observe that, by Langlands' theorem (6.5), if $\nu\left(h_{\alpha}\right)<0$, then $\pi_{\xi, \nu}$ has a unique irreducible subspace, which is not a discrete series. Hence, $\left(\sigma \delta_{\Phi^{+}}, \alpha\right)$ $=\nu\left(h_{\alpha}\right)>0$ and if $\beta \in \Phi_{m}^{+}$, then $\left(\sigma \delta_{\Phi^{+}}, \beta\right)=\left(\Lambda_{\xi}+\delta_{m}, \beta\right)>0$. Therefore $\sigma \delta_{\Phi^{+}}=$ $\delta_{Q}$, with $Q$ as in the statement of the lemma.

REMARK 7.2. In view of the previous results (duality Theorem 6.4 and Lemma 7.1) one can obtain all the embeddings of a discrete series with trivial infinitesimal character in the nonunitary principal series, just knowing all the realizations of such discrete series in principal series $\pi_{\xi, \nu}$ with $\nu>0$.

8. Tensoring with finite dimensional representations. The main results of this section, Theorem 8.5, use Zuckerman's technique of tensoring with finite dimensional representation to show that one can obtain all the embeddings of the discrete series just knowing the embeddings of the discrete series with trivial infinitesimal character. We follow [23] in the terminology "chain" and "composition series". We retain the notation of $\S 2$, we do not need $\operatorname{dim} a=1$. We fix for the rest of the section $\Lambda \in L_{T}$ regular and $P=\{\alpha \in \Delta:(\Lambda, \alpha)>0\}$. We denote as usual by $\left(\pi_{\Lambda}, H^{\Lambda}\right)$ the discrete series representation associated to $\Lambda$, and by $\chi_{\Lambda}$ its infinitesimal character. Let $\mu \in \mathrm{t}_{C}^{*}$ be dominant integral relative to $P$, then $\Lambda+\mu$ corresponds to the discrete series representation $\left(\pi_{\Lambda+\mu}, H^{\Lambda+\mu}\right)$.

The following lemma is due to [22].

LEMMA 8.1. (a) Let $(\pi, F)$ be the irreducible finite dimensional representation of $G$ of lowest weight $-\mu$, then $\left(H_{F}^{\Lambda+\mu} \otimes F\right)_{\chi_{\Lambda}}$ is equivalent to $H_{F}^{\Lambda}$, where $\left(H_{F}^{\Lambda+\mu} \otimes F\right)_{\chi_{\Lambda}}$ is the image of the projection of $H_{F}^{\Lambda+\mu} \otimes F$ according to its infinitesimal character $\chi_{\Lambda}$.

(b) Assume that $\Lambda-\mu$ is regular and $P$ dominant integral. Let $(\pi, F)$ be the irreducible finite dimensional representation of $G$ of highest weight $\mu$. Then $\left(H_{F}^{\Lambda-\mu} \otimes F\right)_{\chi_{\Lambda}}$ is equivalent to $H_{F}^{\Lambda}\left(H^{\Lambda-\mu}\right.$ discrete series $)$.

We will make repeated use of the following lemma.

LEMMA 8.2. Let $W$ be a g-module such that $\operatorname{dim} z \cdot w<\infty$ for each $w \in W$.

Let $W=W_{1} \supset \cdots \supset W_{r+1}=\{0\}$ be a chain such that $W_{j} / W_{j+1}$ has infinitesimal character $\chi_{j}$. Let $\chi=\chi_{i}$ and assume $\chi \neq \chi_{j}$ for $i \neq j$. Let $\left(W_{1}\right)_{\chi}=\{v \in W$ : there exists $k>0, k \in Z$, so that $(Z-\chi(Z))^{k} v=0$ for $\left.Z \in z\right\}$. Then $\left(W_{1}\right)_{\chi} \simeq$ $W_{i} / W_{i+1}$ as g-module.

Proof. Let $P_{\chi}: W_{1} \rightarrow\left(W_{1}\right)_{\chi}$ be the natural projection $\left(W=\Sigma_{\chi \in \hat{\jmath}}(W)_{\chi}\right)$.

(1) $P_{\chi} W_{j}=0$ for $j>i$.

Since $W_{r}$ has infinitesimal character $\chi_{r}$, then $P_{\chi}\left(W_{r}\right)=0$. Assume $P_{\chi}\left(W_{k}\right)$ $=\cdots=P_{k}\left(W_{r}\right)=0$ and prove that $P_{\chi}\left(W_{k-1}\right)=0$ if $k-1>i$.

Let $0 \neq w \in W_{k}$ and denote by $\bar{w}$ the class of $w$ in $W_{k-1} / W_{k}$. Assume $P_{\chi}(w) \neq$ 0 . Then $\bar{w} \neq 0$. Let $k \in Z_{+}$be minimal such that $(Z-\chi(Z))^{k} w=0$ and set $w_{1}=(Z-\chi(Z))^{k-1} w(Z \in z)$. Then $w_{1} \neq 0$ by the minimality of $k$. 
Let $v=\left(Z-\chi_{k-1}(Z)\right) w_{1}$, thus $v \in W_{k}$ and

(2) $(Z-\chi(Z)) v=0$.

If $v=0$, then $\left(Z-\chi_{k-1}(Z)\right) w_{1}=(Z-\chi(Z)) w_{1}$. Since $w_{1} \neq 0$, this last equality would imply $\chi=\chi_{k-1}$. Thus $v \neq 0$ and by the induction hypothesis $P_{\chi}(v)=0$ which contradicts (2). Therefore (1) is true.

(3) $P_{\chi}\left(W_{i}\right)=\cdots=P_{\chi}\left(W_{1}\right)$.

We have the exact sequence of $\mathrm{g}$-modules: $0 \rightarrow W_{i} \rightarrow W_{i-1} \rightarrow W_{i-1} / W_{i} \rightarrow 0$ and hence $0 \rightarrow\left(W_{i}\right)_{\chi} \rightarrow\left(W_{i-1}\right)_{\chi} \rightarrow\left(W_{i-1} / W_{i}\right)_{\chi} \rightarrow 0$ is exact. But $\left(W_{i-1} / W_{i}\right)_{\chi}=0$ since $W_{i-1} / W_{i}$ has infinitesimal character $\chi_{i-1} \neq \chi$. (3) follows by induction.

Using (1) and (3) we have

$$
W_{i} \stackrel{P_{x}}{\rightarrow}\left(W_{1}\right)_{\chi} \rightarrow 0
$$

and $\operatorname{ker} P_{x} \supset W_{i+1}$. We only need to prove that $W_{i+1} \supset \operatorname{ker} P_{x}$. On the other hand, $W_{i}=\left(W_{i}\right)_{x} \oplus \sum_{j \neq i}\left(W_{i}\right)_{x_{j}}$ and $W_{i+1}=\sum_{j \neq i}\left(W_{i+1}\right)_{x_{j}}$.

$$
0 \rightarrow\left(W_{i+1}\right)_{x_{i}} \rightarrow\left(W_{i}\right)_{\chi_{j}} \rightarrow\left(W_{i} / W_{i+1}\right)_{x_{j}} \rightarrow 0
$$

is exact and $\left(W_{i} / W_{i+1}\right)_{\chi_{j}}=0$ for $j \neq i$; hence $\left(W_{i+1}\right)_{\chi_{j}}=\left(W_{i}\right)_{\chi_{j}}$. Now let $v \in W_{i}$ so that $P_{\chi}(v)=0$; then $v \in \Sigma_{j \neq i}\left(W_{i}\right)_{\chi_{j}}=W_{i+1}$. The proof is now complete.

Let $\xi \in \hat{M}$ and $\left(\xi, H^{\xi}\right) \in \xi$. If $\nu \in \mathfrak{a}_{\mathbf{C}}^{*}$ let $\left(\xi \otimes \nu, H^{\xi}\right)$ be the $M A N$ representation defined by $\xi \otimes \nu(\operatorname{man})(v)=e^{(\nu+\rho) 1 g a} \xi(m) v$, for man $\in M A N$, and $v \in H^{\xi}$. Let $(\pi, F)$ be a finite dimensional representation of $G$ and let $\Lambda_{\xi}$ be the highest weight of $\xi$ relative to $\Phi_{m}^{+}$.

LEMMA 8.3. $H^{\xi} \otimes F$ has a composition series given by $H^{\xi} \otimes F=F_{1} \supset \cdots \supset F_{s}$ $\supset\{0\} . F_{i} / F_{i+1}$ is the irreducible $M A N$ module $\xi_{i} \otimes \nu_{i}$, where $\xi_{i} \otimes \nu_{i}$ has a highest weight $\Lambda_{\xi}+\nu+\rho+($ weight of $F)$, relative to $\Phi_{m}^{+}$.

Proof. Let $n^{+}=\Sigma_{\alpha \in \Phi^{+}} \mathfrak{g}_{\alpha}, \mathfrak{g}_{\alpha}$ the root space. Then $\mathfrak{n}^{+} \cap \mathrm{g}=\mathfrak{n}$. Let $\mu_{1}, \ldots, \mu_{d}$ be the weights of $F$ in increasing order and repeated according to their multiplicities. Let $f_{1}, \ldots, f_{d}$ be the corresponding eigenvectors and $v_{\xi}$ the highest weight vector for $\Lambda_{\xi}$. Set $u_{i}=v_{\xi} \otimes f_{i}$ and $F_{i}=\Sigma_{j \geqslant i} U(\mathfrak{b}) u_{j}, j=1, \ldots, d$ and $\mathfrak{b}=\mathrm{m} \oplus \mathrm{a}$ $\oplus$ n.

We have $v_{\xi} \otimes f_{i} \in F_{1}, \forall i$, and $\left(U_{n}(\mathfrak{b}) v_{\xi}\right) \otimes f_{i} \in F_{1}$ by induction on $n$ $\left(\left\{U_{n}(\mathfrak{b})\right\}_{n \geqslant 0}\right.$ canonical filtration of $\left.U(\mathfrak{b})\right)$; hence $H^{\xi} \otimes F=F_{1}$. By the choice of $\Phi^{+}, \xi \otimes \nu \otimes \pi(\mathfrak{n}) F_{i} \subset F_{i+1}$, thus $\left\{F_{i}\right\}_{i=1}^{d}$ are $\mathfrak{n}$ modules. Also, root vectors corresponding to $\Phi_{m}^{+}$carry $F_{i}$ into $F_{i+1}$ and hence $\left\{F_{i}\right\}_{i=1}^{d}$ are $\mathfrak{b}$ modules. It is now clear that if $F_{i} / F_{i+1} \neq 0$, then $M A N$ acts irreducibly on $F_{i} / F_{i+1}$ in the way described.

LEMMA 8.4. Let $\xi \otimes \nu$ be an irreducible finite dimensional representation of MAN on $H^{\xi}$ and let $(\pi, F)$ be a finite dimensional representation of $G$. If $\xi \otimes \nu \otimes \pi_{\mid M A N}$ has a composition series $H^{\xi} \otimes F=F_{1} \supset \cdots \supset F_{k} \supset 0$ with irreducible quotients $\left(\xi_{i} \otimes \nu_{i}, F_{i} / F_{i+1}\right)$ then $\pi_{\xi, \nu} \otimes \pi$ has a corresponding chain, where the respective quotients are infinitesimally equivalent to $\pi_{\xi_{i}, \nu_{i}}(1 \leqslant i \leqslant k)$.

Proof. See [10, Proposition 10.6].

We now go back to the situation $\operatorname{dim} a=1$.

Let $\Phi^{+}$be defined as in $\S 2$ and let $\alpha$ be the real positive root determined by $\Phi^{+}$. 
Let $\operatorname{Ad}(x): \mathrm{t}_{\mathbf{C}} \rightarrow \mathfrak{h}_{\mathbf{C}}$ for $x \in G_{\mathbf{C}}$.

Let $\xi \in \hat{M}$ and $\left(\xi, H^{\xi}\right) \in \xi$. Let $\nu \in a_{\mathbf{C}}^{*}$.

THEOREM 8.5. (a) Let $\mu=\Lambda-\delta_{P}$ and $F_{-\mu}$ be the irreducible $G$ module of lowest weight $-\mu$. If $\pi_{\Lambda}$ is an irreducible component of $\pi_{\xi, \nu}$, then $\pi_{\delta_{p}}$ is an irreducible component of $\pi_{\xi-\tilde{\mu}_{\mid b^{-}}, \nu-\tilde{\mu}_{\mid a}}$, where $-\tilde{\mu}$ is the lowest weight of $F_{-\mu}$, relative to a particular Weyl chamber.

(b) Let $\gamma=\Lambda-\delta_{P}$ and $F_{\gamma}$ be the irreducible $G$ module of highest weight $\gamma$. If $\pi_{\delta_{p}}$ is an irreducible component of $\pi_{\xi, \nu}$, then $\pi_{\Lambda}$ is an irreducible component of $\pi_{\xi+\tilde{\gamma}_{\mid b^{-}}, \nu+\tilde{\gamma}_{\mid a^{2}}}$, where $\tilde{\gamma}$ is the highest weight of $F_{\gamma}$, relative to a particular Weyl chamber.

Proof. We first prove (a). Let $V_{2} \subset V_{1} \subset H^{\xi, \nu}$ be closed invariant subspaces so that $V_{1} / V_{2}$ is infinitesimally equivalent to $H^{\Lambda}$. Lemma 8.1 says that $\left(H_{F}^{\Lambda} \otimes F_{-\mu}\right)_{\chi_{\Lambda-\mu}}$ is g-equivalent to $H^{\delta_{P}}$. On the line of Lemma 7.1, it is very easy to see that $\nu$ is real and different from zero. Thus by the duality theorem we may assume that $\nu\left(h_{\alpha}\right)>0$. By assumption $\chi_{\Lambda(\xi, \nu)}=\chi_{\Lambda}$, hence

$$
\Lambda(\xi, \nu)=\Lambda_{\xi}+\nu+\delta_{m}=\sigma\left(\Lambda \circ \operatorname{Ad}(x)^{-1}\right) \text { for some } \sigma \in W(\Phi) .
$$

Let $P_{1}=\sigma \circ P \operatorname{Ad}(x)^{-1}$, then the regularity of $\Lambda$ says that $\Lambda(\xi, \nu)$ is dominant and regular relative to $P_{1}$.

Let $\tilde{\mu}=\sigma \circ \mu \circ \operatorname{Ad}(x)^{-1}$, then $-\tilde{\mu}$ is the lowest weight of $F_{-\mu}$ relative to $P_{1}$.

(2) $\Lambda(\xi, \nu)-\tilde{\mu}$ is dominant integral and regular relative to $P_{1}$.

Indeed, $\Lambda(\xi, \nu)-\tilde{\mu}=\sigma(\Lambda-\mu) \circ \operatorname{Ad}(x)^{-1}=\delta_{P_{1}}$.

$$
\chi_{\Lambda-\mu}=\chi_{\Lambda(\xi, \nu)-\tilde{\mu}}
$$

Indeed

$$
\begin{aligned}
\chi_{\Lambda-\mu}(Z) & =(\Lambda-\mu)\left(\gamma_{\mathfrak{t}}(Z)\right)=(\Lambda-\mu) \operatorname{Ad}(x)^{-1}\left(\gamma_{\mathfrak{h}}(Z)\right) \\
& =\sigma(\Lambda-\mu) \circ \operatorname{Ad}(x)^{-1}\left(\gamma_{\mathfrak{h}}(Z)\right)=(\Lambda(\xi, \nu)-\tilde{\mu})\left(\gamma_{\mathfrak{h}}(Z)\right)=\chi_{\Lambda(\xi, \nu)-\tilde{\mu}} .
\end{aligned}
$$

(4) $H^{\xi, \nu} \otimes F_{-\mu}$ has a chain by quotients infinitesimally equivalent to $\pi_{\xi+\left.\mu_{i}\right|_{\mathfrak{b}}, \nu+\left.\mu_{i}\right|_{a}}$, where $\mu_{0}=-\tilde{\mu}, \mu_{1}, \ldots, \mu_{k}$ are weights of $F_{-\mu}$ on $\mathfrak{h}_{\mathbf{C}}$. Since $\pi_{\xi+\left.\mu_{0}\right|_{-}, \nu+\mu_{0} l_{a}}$ is in the chain for $H^{\xi, \nu} \otimes F_{-\mu}$ (apply (2) and Lemma 8.4), we get the result combining Lemmas 8.3 and 8.4.

$$
\left(H_{F}^{\xi, \nu} \otimes F_{-\mu}\right)_{\chi_{\Lambda-\mu}} \simeq\left(H_{F}^{\xi, \nu} \otimes F\right)_{\chi_{\Lambda(\xi, \nu)-\tilde{\mu}}} \simeq H^{\xi-\tilde{\mu}_{\mid b-, \nu}-\tilde{\mu}_{\mid b-}}
$$

If we prove that $\chi_{\Lambda(\xi, \nu)+\mu_{1}}=\chi_{\Lambda(\xi, \nu)+\mu_{0}}$ iff $i=0$, then the multiplicity of $\mu_{0}$ being one, together with Lemma 8.2, will give (5).

$\chi_{\Lambda(\xi, \nu)+\mu_{i}}=\chi_{\Lambda(\xi, \nu)+\mu_{0}}$ iff:

(i) $s\left(\Lambda(\xi, \nu)+\mu_{0}\right)=\Lambda(\xi, \nu)+\mu_{i}$ for some $s \in W(\Phi)$. But $\Lambda(\xi, \nu)+\mu_{0}$ is $P_{1}$ dominant integral by (2), thus $s\left(\Lambda(\xi, \nu)+\mu_{0}\right)=\Lambda(\xi, \nu)+\mu_{0}-\Sigma a_{i} \alpha_{i}, a_{i}>0$ and $\alpha_{i} \in P_{1}$. (i) becomes $\mu_{0}-\sum a_{i} \alpha_{i}=\mu_{i}$ which forces $a_{i}=0$, and hence $\mu_{0}=\mu_{i}$ as asserted.

(6) We have the following $g$-equivalences

$$
H_{F}^{\delta_{F}} \simeq\left(H_{F}^{\Lambda} \otimes F_{-\mu}\right)_{\chi_{\Lambda-\mu}} \simeq\left(\left(V_{1} / V_{2}\right)_{F} \otimes F_{-\mu}\right)_{\chi_{\Lambda-\mu}}
$$


By assumption the last term is a subquotient of $\left(H_{F}^{\xi, \nu} \otimes F_{-\mu}\right)_{\chi_{\Lambda-\mu}}$; apply (5) to get the result.

We now prove (b).

We may assume, as before, that $\nu\left(h_{\alpha}\right)>0$. Let $V_{2} \subset V_{1} \subset H^{\xi, \nu}$ so that $V_{1} / V_{2}$ is infinitesimally equivalent to $H^{\delta_{P}}$. By assumption $\chi_{\Lambda(\xi, \nu)}=\chi_{\delta_{p}}$, hence

$$
\Lambda(\xi, \nu)=\Lambda_{\xi}+\nu+\delta_{m}=\sigma\left(\delta_{P} \circ \operatorname{Ad}(x)^{-1}\right) \text { for some } \sigma \in W(\Phi) .
$$

Let $P_{1}=\sigma \circ P \circ \operatorname{Ad}(x)^{-1}$ (note $P_{1} \supset \alpha$ and $\Phi_{m}^{+}$), then the regularity of $\delta_{P}$ says that $\Lambda(\xi, \nu)$ is dominant and regular relative to $P_{1}$. Let $\tilde{\gamma}=\sigma \circ \gamma \circ \operatorname{Ad}(x)^{-1}$, then $\tilde{\gamma}$ is the highest weight of $F_{\gamma}$ relative to $P_{1}$. Since $\Lambda(\xi, \nu)+\tilde{\gamma}=\sigma\left(\delta_{P}+\gamma\right) \circ \operatorname{Ad}(x)^{-1}$ it is clear that

(2') $\Lambda(\xi, \nu)+\tilde{\gamma}$ is dominant integral and regular relative to $P_{1}$.

$$
\chi_{\Lambda(\xi, \nu)+\tilde{\gamma}}=\chi_{\delta_{p}+\gamma}
$$

Indeed, by $\left(1^{\prime}\right)$,

$$
\begin{aligned}
\chi_{\Lambda(\xi, \nu)+\tilde{\gamma}}(Z) & =(\Lambda(\xi, \nu)+\tilde{\gamma})\left(\gamma_{\mathfrak{h}}(Z)\right) \\
& =\sigma\left(\delta_{P}+\gamma\right) \circ \operatorname{Ad}(x)^{-1}\left(\gamma_{\mathfrak{h}}(Z)\right)=\sigma\left(\delta_{P}+\gamma\right) \gamma_{\mathfrak{t}}(Z)=\chi_{\delta_{p}+\gamma}(Z) .
\end{aligned}
$$

Combining Lemmas 8.3 and 8.4 we have

(4') $H^{\xi, \nu} \otimes F_{\gamma}$ has a chain by quotients infinitesimally equivalent to $\pi_{\xi+\gamma_{i l_{b}}, \nu+\gamma_{i \mid a}}$, where $\gamma_{1}=\tilde{\gamma}, \gamma_{2}, \ldots, \gamma_{k}$ are weights of $F_{\gamma}$ on $\mathfrak{h}_{\mathbf{c}}$.

In view of $\left(3^{\prime}\right)$,

$$
\begin{gathered}
\left(H^{\xi, \nu} \otimes F_{\gamma}\right)_{\chi_{\delta_{p}+\gamma}}=\left(H^{\xi, \nu} \otimes F_{\gamma}\right)_{\chi_{\Lambda(\xi, \nu)+\tilde{\gamma}}}, \\
\chi_{\Lambda(\xi, \nu)+\gamma_{i}}=\chi_{\Lambda(\xi, \nu)+\tilde{\gamma}} \quad \text { iff } \gamma_{i}=\tilde{\gamma} .
\end{gathered}
$$

Indeed, $\chi_{\Lambda(\xi, \nu)+\gamma_{i}}=\chi_{\Lambda(\xi, \nu)+\tilde{\gamma}}$ implies

$$
\Lambda(\xi, \nu)+\gamma_{i}=s(\Lambda(\xi, \nu)+\tilde{\gamma}) \text { for some } s \in w(\Phi) .
$$

But $\Lambda(\xi, \nu)+\tilde{\gamma}$ is dominant relative to $P_{1}$, thus, $s(\Lambda(\xi, \nu)+\tilde{\gamma})=\Lambda(\xi, \nu)+\tilde{\gamma}-$ $\sum r_{i} \alpha_{i}, r_{i} \geqslant 0$ and $\alpha_{i} \in P_{1}$. But then $\gamma_{i}=\tilde{\gamma}-\sum r_{i} \alpha_{i}$. This implies $r_{i}=0$ since $\gamma$ is the highest weight. Thus $\gamma_{i}=\tilde{\gamma}$ as asserted and (6') follows.

(7') The multiplicity of $\tilde{\gamma}$ being one, together with Lemma 8.2 gives

$$
\left(H^{\xi, \nu} \otimes F_{\gamma}\right)_{x_{\delta_{p}+\gamma}}=H^{\xi+\tilde{\gamma}_{b^{-}}-, \nu+\tilde{\gamma}_{b^{-}}-} .
$$

Since $H^{\Lambda}$ is infinitesimally equivalent to $\left(H^{\delta_{P}} \otimes F_{\gamma}\right)_{x_{\delta_{p}+\gamma}}$, then $\left(7^{\prime}\right)$ gives the result.

REMARK 8.6. In the previous theorem, it is implicit that the realizations of the discrete series $\pi_{\Lambda}$ and $\pi_{\delta_{p}}$ correspond. That is to say, if $\pi_{\delta_{p}}$ is a subspace (quotient), then $\pi_{\Lambda}$ is a subspace (quotient).

Let us assume that $\pi_{\delta_{p}}$ is a subspace or a quotient of $\pi_{\xi, \nu}$. Then $\pi_{\Lambda}$ is a subspace or a quotient of $\pi_{\xi_{1}, \nu_{1}}$. The computation of $\Lambda\left(\xi_{1}, \nu_{1}\right)$ is contained in the proof of the theorem, but we explicitly point it out.

$\Lambda(\xi, \nu)=\delta_{\tilde{P}}$ for some $\tilde{P}$ positive containing $\alpha$ and $\Phi_{m}^{+}$(cf. Lemma 7.1). Let $\tilde{\Lambda}$ be equal to $\Lambda$ computed relative to $\tilde{P}$. Then $\tilde{\gamma}=\Lambda-\delta_{P}$ and $\Lambda\left(\xi_{1}, \nu_{1}\right)=\Lambda(\xi, \nu)+\tilde{\gamma}$. Thus all we need are explicit tables for the embeddings of the various $\pi_{\delta_{p}}$ 's. We will give them in $\S 18$. 
9. Character identities. Let the assumption be as in $\$ 2$. Let $\gamma$ be a noncompact root, positive relative to some system of positive roots for $\Delta$. Choose the root vectors $X_{\gamma}, X_{-\gamma}$ so that $X_{\gamma}+X_{-\gamma} \in \mathfrak{p}$ and let $\mathfrak{a}=\mathbf{R}\left(X_{\gamma}+X_{-\gamma}\right)$. Let $u_{\gamma}=$ $\exp \pi\left(X_{\gamma}-X_{-\gamma}\right) / 4$, then the Cayley transform Ad $u_{\gamma}: \mathfrak{t}_{\mathbf{C}} \rightarrow\left(\mathfrak{h}^{-}+\mathfrak{a}\right)_{\mathbf{C}}$. Also $\Phi=$ $\Delta \circ \operatorname{Ad~} u_{\gamma}^{-1}$ and $\Phi_{m}=\left\{\alpha \circ \operatorname{Ad} u_{\gamma}^{-1}:(\alpha, \gamma)=0\right\}$. Let $\Phi^{+}$be a system of positive roots for $\Phi$ defined as in $\S 2$. Fix $\Phi_{m}^{+}$and $\Lambda^{+}$consistently. Let $P=\Phi^{+} \circ$ Ad $u_{\gamma}$, then $P$ is positive for $\Delta$ and $\gamma \in P$. Let $\alpha=\gamma \circ$ Ad $u_{\gamma}^{-1}$, then $\alpha$ is the unique real root determined by $\Phi^{+}$. We fix $\tau \in \mathrm{t}_{C}^{*}$ a regular and dominant integral form relative to $P$. Let $\sigma_{\tau}^{G}$ be the irreducible finite dimensional representation of $G$ of highest weight $\tau-\delta_{P}$, relative to $P$.

Set $W_{1}=\left\{\omega \in W(\Phi): \omega^{-1} \beta>0\right.$ for each $\beta \in \Phi_{m}^{+}$or $\left.\beta=\alpha\right\}$. Let $\Lambda=$ $\tau \circ$ Ad $u_{\gamma}^{-1}$. If $\omega \in W_{1}$, let $\sigma_{\omega}$ be the irreducible finite dimensional representation of $M$ of highest weight $\left.\omega \Lambda\right|_{\mathfrak{h}^{-}}-\delta_{m}$, relative to $\Phi_{m}^{+}$, and let $\nu_{\omega}$ be the element of $a^{*}$ defined by $\nu_{\omega}(H)=\omega \Lambda(H)$. Consider $\left\{\pi_{\sigma_{\omega}}, \nu_{\omega}\right\}_{\omega \in W_{1}}$. Notice that

(1) $\left(\nu_{\omega}, \alpha\right)=(\omega \Lambda, \alpha)=\left(\Lambda, \omega^{-1} \alpha\right)>0$ by regularity of $\Lambda$, i.e. $\nu_{\omega}>0$.

(2) $\chi_{\sigma_{\omega}, \nu_{\omega}}=\chi_{\tau}$.

Indeed

$$
\begin{aligned}
\chi_{\sigma_{\omega}, \nu_{\omega}}(Z) & =\left(\left.\omega \Lambda\right|_{\mathfrak{h}^{-}}-\delta_{m}+\delta_{m}+\nu_{\omega}\right)\left(\gamma_{\mathfrak{h}^{-}+\mathfrak{a}}(Z)\right) \\
& =\omega \Lambda\left(\gamma_{\mathfrak{h}^{-}+\mathfrak{a}}(Z)\right)=\Lambda\left(\gamma_{\mathfrak{h}^{-}+\mathfrak{a}}(Z)\right) \tau\left(\gamma_{\mathfrak{t}}(Z)\right)=\chi_{\boldsymbol{r}}, \quad \text { for } Z \in \mathfrak{z} .
\end{aligned}
$$

The following theorem is due to [14] and [21]. For a direct proof see also [1].

THEOREM 9.1.

$$
(-1)^{q} \sum_{s \in W / W_{k}} \Theta_{s \tau}=\chi\left(\sigma_{\tau}^{G}\right)-\sum_{\omega \in W_{1}} \operatorname{det}(\omega) \Theta_{\sigma_{\omega}, \nu_{\omega}}
$$

$\left(\Theta_{s \tau}\right.$ is the character of the discrete series $\pi_{\sigma \tau}, q=\frac{1}{2} \operatorname{dim} G / K$ and $\chi\left(\sigma_{\tau}^{G}\right)$ is the character of $\sigma_{\tau}^{G}$.)

REMARK 9.2. It is immediate that the sum of the right-hand side of (3) contains the characters of all the principal series with a parameter real and for which (1) and (2) are true.

Let $\hat{G}_{d}$ denote the set of the discrete series of $G$.

Corollary 9.3. Let $G=\operatorname{SU}(n, 1), \operatorname{Spin}(2 n, 1), \operatorname{Sp}(n, 1)$ or $F_{4}$. If $\omega \in \hat{G}_{d}$ then there are an odd number of subquotients of $\omega$ in $\left\{\pi_{\xi, \nu}\right\}_{\xi \in \hat{M}, \nu \text { real, }(\nu, \lambda)>0}$.

Proof. From the argument of tensoring with finite dimensional representation of $\S 8$, we may assume that $\chi_{\omega}=0\left(\chi_{\omega}\right.$ infinitesimal character of $\left.\omega\right)$. If $\eta \in \hat{G}_{d}$, write $\Theta_{\eta}$ for its character. We can rewrite (3) as

$$
(-1)^{q} \sum_{\substack{n \in \hat{G}_{d} \\ \chi_{\eta}=0}} \Theta_{\eta}=1+\sum_{i=1}^{n} \varepsilon_{i} \Theta_{2}
$$

where $\varepsilon_{i}= \pm 1, \xi_{i} \in \hat{M}, \nu_{i}$ real, $\nu_{i}>0, \xi_{i}, \nu_{i}$ all distinct. 
On the other hand the characters of inequivalent irreducible representations are linearly independent and a nontrivial unitary representation of a simple noncompact group is infinite dimensonal (cf. [20, I, ex. 4]). Hence

$$
\Theta_{\eta}=\sum_{i=1}^{n} \varepsilon_{i} m_{i} \Theta_{\eta}, \quad \text { where } m_{i}=1 \text { if } \eta \text { is a subquotient of } \pi_{\xi, \nu_{i}}
$$

(cf. Theorem 17.9 for the fact that $m_{i}=1$ ) and $m_{i}=0$ if $\eta$ is not a subquotient of $\pi_{\xi_{i}, \nu_{i}}$

By Remark 9.2, $\sum m_{i}$ gives the total number of subquotients, hence (4) forces $1=\sum_{i=1}^{n} \varepsilon_{i} m_{i}=\sum_{i=1}^{n} m_{i} \bmod 2$. Thus $\sum m_{i}=1 \bmod 2$, as asserted.

\section{Branching theorem.}

10.1. From now on we will assume that $\operatorname{dim} a=1$ and that $G \neq \operatorname{Sl}(2, \mathbf{R})$.

Then $G$ is up to isomorphism one of the following groups:

(1) $\operatorname{Spin}(2 n, 1), n \geqslant 2$,

(2) $\mathrm{SU}(n, 1), n \geqslant 2$,

(3) $\operatorname{Sp}(n, 1), n \geqslant 2$,

(4) $F_{4}$ the analytic group corresponding to the real form $g=f_{4(-20)}$ of $g_{C}=f_{4}$, with character -20 .

The restrictions on the indices are set in order to avoid overlapping, since $\operatorname{so}(2,1) \simeq \operatorname{sl}(2, \mathbf{R}) \simeq \operatorname{su}(1,1)$ and $\operatorname{so}(4,1) \simeq \operatorname{sp}(1,1)$. The Cartan involution is denoted by $\theta$ and bar denotes conjugation of $g_{C}$ with respect to $g$. Then $X \rightarrow \theta \bar{X}$ is the conjugation of $g_{\mathbf{C}}$ with respect to the compact form $\oplus i \mathrm{p}$.

We shall need to make computation with root vectors, and we fix a normalization of them. Namely by [7] we can select root vectors $X_{\alpha}$ in such a way that $B\left(X_{\alpha}, X_{-\alpha}\right)=2 /(\alpha, \alpha)$ and $\theta \bar{X}_{\alpha}=-X_{\alpha}$. Then it follows that $H_{\alpha}$ defined by $H_{\alpha}=\left[X_{\alpha}, X_{-\alpha}\right]$ satisfies $\alpha\left(H_{\alpha}\right)=2$ and that $X_{\alpha}+X_{-\alpha}, i\left(X_{\alpha}-X_{-\alpha}\right)$ are in $g$ if $\alpha$ is noncompact; $X_{\alpha}-X_{-\alpha}, i\left(X_{\alpha}+X_{-\alpha}\right)$ are in $g$ if $\alpha$ is compact.

If $U$ is a compact connected Lie group and $B$ a maximal torus of $U$, denote by $\mathfrak{b}$ and $\mathfrak{u}$ the Lie algebras of $B$ and $U$, respectively. Then $\mathfrak{b}=z_{u} \oplus \mathfrak{b}_{1}$ where $z_{u}$ is the center of $\mathfrak{u}$ and $\mathfrak{b}_{1}$ is a Cartan subalgebra of $[\mathfrak{u}, \mathfrak{u}]$.

It is well known [17] that $\hat{U}$, the set of all equivalence classes of irreducible finite dimensional (complex) representations of $U$, is in bijective correspondence with $D_{U}=\left\{\lambda\right.$ linear form on $\mathfrak{b}_{\mathbf{c}}$ such that $(1) \lambda\left(\Gamma_{U}\right) \subset 2 \pi i Z$ for $\Gamma_{U}=\{X \in \mathfrak{b}$ : $\exp X=$ $e$; (2) $\lambda_{l_{\left(b_{1}\right) c}}$ is dominant integral relative to some choice of positive roots $\}$.

If $\lambda \in D_{U}$ we denote by $\left(\pi_{\lambda}, V^{\lambda}\right)$ the $U$ representation parametrized by $\lambda$. If $H$ is a compact connected subgroup of $U$ and $S \subset B$ is a maximal torus, then for $\lambda \in D_{U}, \mu \in D_{H}$ we define $m_{\lambda}(\mu)$ to be the multiplicity with which the finite dimensional (complex) representation of $H, \pi_{\mu}$, appears in $\pi_{\left.\lambda\right|_{H}}$.

We now go back to our situation; we retain the notation of $\S 2$. Fix $\Delta^{+}$a system of positive roots for $\Delta$ and let $\Delta_{k}^{+}=\Delta_{k} \cap \Delta^{+}$. Let $\lambda$ be a positive noncompact root. Then $\mathfrak{a}=\mathbf{R}\left(X_{\lambda}+X_{-\lambda}\right)$ is a maximal abelian subalgebra of $\mathfrak{p}$. Let $\mathfrak{h}^{-}=\mathbf{R} i H_{\alpha}$, where $\alpha \in \Delta$ and $(\alpha, \lambda)=0$. The standard Cayley transform relative to $\lambda$ is $\operatorname{Ad}\left(u_{\lambda}\right)$, where $u_{\lambda}=\exp \left((\pi / 4)\left(X_{\lambda}-X_{-\lambda}\right)\right)$. 
Then $\operatorname{Ad}\left(u_{\lambda}\right)$ carries $\mathrm{t}_{\mathbf{C}}$ to $\mathfrak{h}_{\mathbf{C}}$ and hence $\Phi=\Delta \circ \operatorname{Ad}\left(u_{\lambda}\right)^{-1}$. In particular the roots of $\Phi_{m}$ are of the form $\gamma=\beta \circ \operatorname{Ad}\left(u_{\lambda}\right)^{-1}$, with $\beta \in \Delta$ and $\beta$ orthogonal to $\lambda$. If $\left\{\alpha_{1}, \ldots, \alpha_{m}\right\}$ are simple for $\Delta^{+}$, denote by $\left\{\beta_{1}, \ldots, \beta_{m}\right\}$ the roots of $\Phi$ defined by $\beta_{i}=\alpha_{i} \circ \operatorname{Ad}\left(u_{\lambda}\right)^{-1}$. Set $\bar{\alpha}_{i}=\beta_{i} \circ \operatorname{Ad}\left(u_{\lambda}\right)_{\left.\right|_{b \bar{c}}}^{-1}=\alpha_{\left.i\right|_{h \bar{c}}}$.

In what follows, we will fix for each group of type (1)-(4) a system of positive roots $\Phi^{+}$for $\Phi$ and the real root $\alpha$ for $\Phi^{+} . \Phi_{m}^{+}$and $\Lambda^{+}$will be defined consistently as in $\S 2$. The notion of dominance for $K$ and $M$ will be relative to $\Delta_{k}^{+}$and $\Phi_{m}^{+}$.

The following branching theorems are proved in [1]. They are obtained using results of [13] and classical branching theorems.

10.2. Let $G=\operatorname{Spin}(2 n, 1)$, then $g=\operatorname{so}(2 n, 1)$.

Let

$$
\begin{aligned}
& \mathfrak{f}=\left\{\left(\begin{array}{ll}
A & 0 \\
0 & 0
\end{array}\right), A \in \operatorname{so}(2 n)\right\} \quad \text { and } \\
& \mathrm{t}=\left\{\left[\begin{array}{ccc}
0 & A & \\
-A^{t} & 0 & \\
& & 0
\end{array}\right] \in \operatorname{so}(2 n, 1) \text { where } A \text { is an } n \times n \text { diagonal matrix }\right\}
\end{aligned}
$$

We take the simple roots of $\Delta^{+}$, labelled as follows:

$$
\Delta^{+}: \underset{\alpha_{1}}{\stackrel{\alpha}{\alpha_{2}}} \stackrel{\circ}{\circ} \cdots \underset{\alpha_{n-1}}{\circ} \Rightarrow \underset{\alpha_{n}}{\circ}, \quad \text { with } \alpha_{j}=\varepsilon_{j}-\varepsilon_{j+1}, 1<j \leqslant n-1 \text {, }
$$

and $\alpha_{n}=\varepsilon_{n}$ as in Bourbaki [2]. Then

$$
\Delta_{k}^{+}: \underset{\alpha_{1}}{\circ} \cdots<_{\alpha_{n-2}}^{\alpha_{n-1}}<_{\alpha_{n-1}+2 \alpha_{n}}^{\circ}
$$

We take $\lambda=\alpha_{n}$, then $a=\mathbf{R}\left(X_{\alpha_{n}}+X_{-\alpha_{n}}\right)$. Fix

$$
\Phi^{+}: \underset{\gamma_{1}}{\circ} \cdots \underset{\gamma_{n-1}}{\circ} \Rightarrow \underset{\gamma_{n}}{\circ}
$$

with

$$
\begin{aligned}
& \gamma_{1}=-\beta_{1}-\cdots-\beta_{n-1}, \\
& \gamma_{i}=\beta_{i-1}, \quad 2 \leqslant i \leqslant n-1, \\
& \gamma_{n}=\beta_{n-1}+\beta_{n} .
\end{aligned}
$$

Define $\tilde{\varepsilon}_{i}$ in terms of the dual basis $\lambda_{i}$ of the $\gamma_{i}$ 's,

$$
\left(2 \frac{\left(\lambda_{i}, \gamma_{i}\right)}{\left(\gamma_{j}, \gamma_{j}\right)}=\delta_{i j}\right) \text {, }
$$

then $\gamma_{i}=\tilde{\varepsilon}_{i}-\tilde{\varepsilon}_{i+1}, 1 \leqslant i \leqslant n-1, \gamma_{n}=\tilde{\varepsilon}_{n}$. Also

$$
\Phi_{m}^{+}: \underset{\gamma_{2}}{\stackrel{\gamma_{3}}{\circ}} \underset{\gamma_{n-1}}{\circ} \cdots \underset{\gamma_{n}}{\circ}
$$


or in terms of the $\alpha_{i}$ 's,

$$
\Phi_{m}^{+}: \underset{\bar{\alpha}_{1}}{\circ} \ldots \underset{\bar{\alpha}_{n-2}}{\circ} \underset{\bar{\alpha}_{n-1}+\bar{\alpha}_{n}}{\circ}
$$

The real root $\alpha$ for $\Phi^{+}$is $\alpha=\gamma_{1}+\cdots+\gamma_{n}=\tilde{\varepsilon}_{i}$. Since $K \simeq \operatorname{Spin}(2 n)$ and $M \simeq \operatorname{Spin}(2 n-1)$ we have

$$
\begin{aligned}
& D_{K}=\left\{\lambda=\sum_{i=1}^{n} a_{i} \varepsilon_{i} ; a_{1} \geqslant \ldots \geqslant a_{n-1} \geqslant\left|a_{n}\right| \geqslant 0,\right. \\
&\left.a_{i}-a_{j} \in \mathbf{Z} \text { and } 2 a_{i} \in \mathbf{Z} \text { for } i, j=1, \ldots, n\right\} . \\
& D_{M}=\left\{\mu=\sum_{i=1}^{n-1} b_{i} \varepsilon_{i} ; b_{1} \geqslant \ldots \geqslant b_{n-1} \geqslant 0,\right. \\
&\left.b_{i}-b_{j} \in \mathbf{Z} \text { and } 2 b_{i} \in \mathbf{Z} \text { for } i, j=1, \ldots, n-1\right\} .
\end{aligned}
$$

THEOREM 10.3. (Notation as in 10.2.) Let $\lambda=\sum_{i=1}^{n} a_{i} \varepsilon_{i} \in D_{K}$ and $\mu=\sum_{i=1}^{n-1} b_{i} \varepsilon_{i}$ $\in D_{M}$. Then $m_{\lambda}(\mu)=0$ or 1 .

If $a_{i}-b_{j} \notin \mathbf{Z}$, then $m_{\lambda}(\mu)=0$. If $a_{i}-b_{j} \in \mathbf{Z}$, then $m_{\lambda}(\mu)=1$ iff $a_{1}>b_{1}$ $\geqslant \cdots \geqslant a_{n-1} \geqslant b_{n-1} \geqslant\left|a_{n}\right|$.

10.4. Let $G=\operatorname{Su}(n, 1), n \geqslant 2$, then $g=\operatorname{su}(n, 1)$. Let

$$
\mathfrak{f}=\left\{\left(\begin{array}{ll}
A & 0 \\
0 & d
\end{array}\right), A \in u(n), d \in u(1) \text { and } \operatorname{tr} A+d=0\right\},
$$

then $\simeq \operatorname{su}(n) \times \mathbf{R}$.

Let $\mathrm{t}$ be the diagonal matrices in $\operatorname{su}(n, 1)$. We take the simple roots of $\Delta^{+}$ labelled as follows:

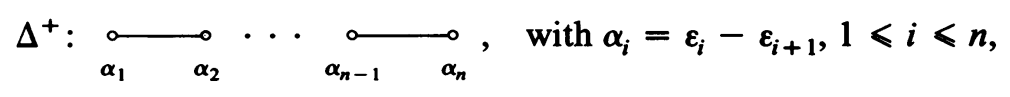

as in Bourbaki [2]. Then

$$
\Delta_{k}^{+}: \underset{\alpha_{1}}{\circ} \cdots \underset{\alpha_{n-1}}{\circ} .
$$

We take $\lambda=\varepsilon_{1}-\varepsilon_{n+1}$ and $a=\mathbf{R}\left(X_{\lambda}+X_{-\lambda}\right)$. Fix

$$
\Phi^{+}: \begin{array}{ccc}
\circ & \cdots & \circ \\
\gamma_{1} & & \gamma_{n}
\end{array} \text { with } \gamma_{i}=\beta_{i}, 1<i<n .
$$

Define $\tilde{\varepsilon}_{i}$ in terms of the dual basis $\lambda_{i}$ of the $\alpha_{i}$ 's, then $\gamma_{i}=\tilde{\varepsilon}_{i}-\tilde{\varepsilon}_{i+1}$.

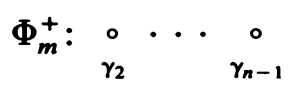

or in terms of the $\alpha_{i}$ 's,

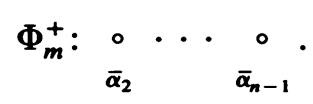


The real root $\alpha$ for $\Phi^{+}$is $\alpha=\gamma_{1}+\cdots+\gamma_{n}=\tilde{\varepsilon}_{1}-\tilde{\varepsilon}_{n+1}$. We have

$$
\begin{gathered}
D_{K}=\left\{\lambda=\sum_{i=1}^{n+1} a_{i} \varepsilon_{i} ; a_{1} \geqslant \ldots \geqslant a_{n}, a_{i} \in \mathbf{Z}, 1 \leqslant i \leqslant n+1\right\} . \\
D_{M}=\left\{\mu=b_{0}\left(\varepsilon_{1}+\varepsilon_{n+1}\right)+\sum_{i=2}^{n} b_{i} \varepsilon_{i}, b_{2} \geqslant \cdots \geqslant b_{n},\right. \\
\left.2 b_{0} \in \mathbf{Z}, b_{i} \in \mathbf{Z} \text { for } i=2, \ldots, n\right\} .
\end{gathered}
$$

THEOREM 10.5. (Notation as in 10.4.) Let $\lambda=\sum_{i=1}^{n+1} a_{i} \varepsilon_{i} \in D_{K}$ and $\mu=$ $b_{0}\left(\varepsilon_{1}+\varepsilon_{n+1}\right)+\sum_{i=2}^{n} b_{i} \varepsilon_{i} \in D_{M}$.

Let $b_{i} \in \mathbf{Z}$ be defined by $\sum_{i=1}^{n} b_{i}=\sum_{i=1}^{n} a_{i}$. Then $m_{\lambda}(\mu)=1$ iff $a_{1} \geqslant b_{2}$ $\geqslant \cdots \geqslant b_{n} \geqslant a_{n}$ and $b_{0}=\left(b_{1}+a_{n+1}\right) / 2$. Otherwise $m_{\lambda}(\mu)=0$.

10.6. Let $G=\operatorname{Sp}(n, 1), n \geqslant 2$, then $g=\operatorname{sp}(n, 1)$. Let

$$
\left.\mathfrak{f}=\left\{\begin{array}{cccc}
-C & 0 & D & 0 \\
0 & t & 0 & s \\
-\bar{D} & 0 & \bar{C} & 0 \\
0 & -\bar{s} & 0 & \bar{t}
\end{array}\right\} \quad \begin{array}{l}
C, D \text { complex } n \times n \text { matrices } C \in u(n), \\
D \text { symmetric, } t \in u(1) \text { and } s \in \mathrm{C}
\end{array}\right\} .
$$

Let $\mathrm{t}$ be the set of the diagonal matrices in $\mathrm{g}$. We take the simple roots of $\Delta^{+}$to be given by

$$
\Delta^{+}: \underset{\alpha_{1}}{\circ} \cdots \underset{\alpha_{n}}{\circ} \Leftarrow \stackrel{\circ}{\circ} \text {, with } \alpha_{i}=\varepsilon_{i+1}-\varepsilon_{i+1}, 1 \leqslant i \leqslant n \text {, and } \alpha_{n+1}=2 \varepsilon_{n+1}
$$

in Bourbaki [2]. Then

$$
\Delta_{k}^{+}: \underset{\alpha_{1}}{\circ} \cdots \underset{\alpha_{n-1}}{\circ} \underset{2 \alpha_{n}+\alpha_{n+1}}{\circ} \stackrel{\circ}{\circ} \alpha_{n+1}^{\circ} .
$$

We take $\lambda=\varepsilon_{1}-\varepsilon_{n+1}$ and $a=\mathbf{R}\left(X_{\lambda}+X_{-\lambda}\right)$. Fix

$$
\Phi^{+}: \underset{\gamma_{1}}{\circ} \cdots \underset{\gamma_{n}}{\circ} \stackrel{\circ}{\circ}
$$

where

$$
\begin{aligned}
\gamma_{1} & =\beta_{1}+\cdots+\beta_{n+1}, \quad \gamma_{2}=-\beta_{2}-\cdots-\beta_{n+1}, \\
\gamma_{i} & =\beta_{i-1}, \quad 3 \leqslant i \leqslant n, \\
\gamma_{n+1} & =2 \beta_{n}+\beta_{n+1} .
\end{aligned}
$$

Define $\tilde{\varepsilon}_{i}$ in terms of the dual basis $\lambda_{i}$ of the $\gamma_{i}$ 's; then $\gamma_{i}=\tilde{\varepsilon}_{i}-\tilde{\varepsilon}_{i+1}, 1 \leqslant i \leqslant n$, and $\gamma_{n+1}=2 \tilde{\varepsilon}_{n+1}$.

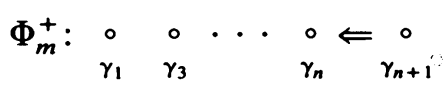

or in terms of the $\alpha_{i}$ 's,

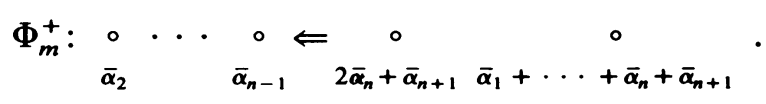


The real root $\alpha$ for $\Phi^{+}$is $\alpha=\gamma_{1}+2 \gamma_{2}+\cdots+2 \gamma_{n}+\gamma_{n+1}=\tilde{\varepsilon}_{1}+\tilde{\varepsilon}_{2}$. We have

$$
\begin{gathered}
D_{K}=\left\{\lambda=\sum_{i=1}^{n+1} a_{i} \varepsilon_{i}, a_{1} \geqslant \cdots \geqslant a_{n} \geqslant 0, a_{n+1} \geqslant 0, a_{i} \in \mathbf{Z}, 1 \leqslant i<n+1\right\} . \\
D_{M}=\left\{\mu=b_{0}\left(\varepsilon_{1}+\varepsilon_{n+1}\right)+\sum_{i=2}^{n} b_{i} \varepsilon_{i}, b_{2} \geqslant \cdots \geqslant b_{n} \geqslant 0,\right. \\
\left.2 b_{0} \in \mathbf{Z}_{+} \text {and } b_{i} \in \mathbf{Z}, i=2, \ldots, n\right\} .
\end{gathered}
$$

THEOREM 10.7. (Notation as in 10.6.) Let $\lambda=\sum_{i=1}^{n+1} a_{i} \varepsilon_{i} \in D_{K}$ and $\mu=$ $b_{0}\left(\varepsilon_{1}+\varepsilon_{n+1}\right)+\sum_{i=2}^{n} b_{i} \varepsilon_{i} \in D_{M}$. Define

$$
\begin{aligned}
A_{1} & =a_{1}-\max \left(a_{2}, b_{2}\right), \\
A_{2} & =\min \left(a_{2}, b_{2}\right)-\max \left(a_{3}, b_{3}\right), \\
\vdots & \\
A_{n-1} & =\min \left(a_{n-1}, b_{n-1}\right)-\max \left(a_{n}, b_{n}\right), \\
A_{n} & =\min \left(a_{n}, b_{n}\right) .
\end{aligned}
$$

Then $m_{\lambda}(\mu)=0$ unless:

(1) $a_{i} \geqslant b_{i+1}, i=1, \ldots, n-1$,

(2) $b_{i} \geqslant a_{i+1}, i=2, \ldots, n-1$, and

(3) $b_{0}=\left(a_{n+1}+b_{1}-2 j\right) / 2$ for some $j=0, \ldots, \min \left(a_{n+1}, b_{1}\right)$,

where $b_{1}$ satisfies $b_{1} \in \mathbf{Z}_{+}$and $\sum_{i=1}^{n}\left(a_{i}+b_{i}\right) \in 2 Z$. If these conditions hold then: $m_{\lambda}(\mu)=\sum_{b_{1} \text { satisfying (3) }} \tilde{m}_{\lambda}(\mu)$ where

$$
\tilde{m}_{\lambda}(\mu)=\sum_{L \subset\{1, \ldots, n\}}(-1)^{|L|}\left(\begin{array}{c}
n-2-|L|+\frac{1}{2}\left(-b_{1}+\sum_{1}^{n} A_{i}\right)-\sum_{i \in L} A_{i} \\
n-2
\end{array}\right) .
$$

$\left(|L|\right.$ is the cardinality of $L$ and $\left(\begin{array}{l}x \\ y\end{array}\right)$ is defined to be $=0$ if $\left.x-y \in \mathbf{Z}_{+}\right)$.

10.8. Let $G=F_{4}$, then $g=f_{4(-20)}, \mathfrak{f}=\operatorname{so}(9)$ and $K=\operatorname{Spin}(9)$. Let

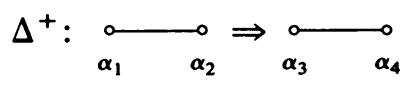

be a choice of simple roots for $\Delta^{+}$. Define $\varepsilon_{1}, \varepsilon_{2}, \varepsilon_{3}, \varepsilon_{4}$ in terms of the dual basis of the $\alpha_{i}$ 's (cf. Bourbaki [2]). Then $\alpha_{1}=\varepsilon_{2}-\varepsilon_{3}, \alpha_{2}=\varepsilon_{3}-\varepsilon_{4}, \alpha_{3}=\varepsilon_{4}, \alpha_{4}=$ $\frac{1}{2}\left(\varepsilon_{1}-\varepsilon_{2}-\varepsilon_{3}-\varepsilon_{4}\right)$. We have

$$
\Delta_{k}^{+}: \quad \begin{array}{cccc}
\alpha_{2}+2 \alpha_{3}+2 \alpha_{4} & \alpha_{1} & \alpha_{2} & \alpha_{3}
\end{array}
$$

We choose $\lambda=\alpha_{4}$ so that $a=\mathbf{R}\left(X_{\alpha_{4}}+X_{-\alpha_{4}}\right)$ and $\mathfrak{m}=\operatorname{so}(7)$. Fix

$$
\Phi^{+}: \begin{array}{rrrr}
\gamma_{1} & \gamma_{2} & r_{3} & r_{4}
\end{array}
$$


with $\gamma_{1}=\beta_{2}, \gamma_{2}=\beta_{1}, \gamma_{3}=\beta_{2}+2 \beta_{3}+\beta_{4}$ and $\gamma_{4}=-\left(\beta_{1}+2 \beta_{2}+3 \beta_{3}+\beta_{4}\right)$. Define $\tilde{\varepsilon}_{i}$ in terms of the dual basis of the $\gamma_{i}$ 's, then $\gamma_{1}=\tilde{\varepsilon}_{2}-\tilde{\varepsilon}_{3}, \gamma_{2}=\tilde{\varepsilon}_{3}-\tilde{\varepsilon}_{4}$, $\gamma_{3}=\tilde{\varepsilon}_{4}$ and $\gamma_{4}=\frac{1}{2}\left(\tilde{\varepsilon}_{1}-\tilde{\varepsilon}_{2}-\tilde{\varepsilon}_{3}-\tilde{\varepsilon}_{4}\right)$.

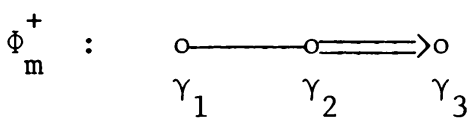

or in terms of the $\alpha_{i}$ 's,

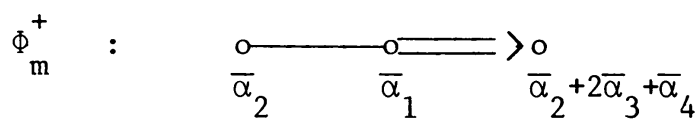

Let $K_{1}$ be the subgroup of $K$ isomorphic to $\operatorname{Spin}(8)$ so that a system of positive roots for $\mathfrak{f}_{1} \simeq \operatorname{so}(8)$ is given in the following way:

$\left(f_{1}\right)_{C} \quad:$

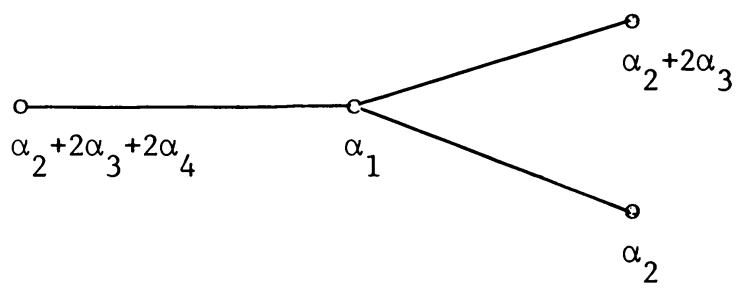

Define an automorphism $\phi$ of $\left(\mathfrak{f}_{1}\right)_{\mathbf{c}}$ which preserves the roots and such that $\mathfrak{m}_{1}=\phi\left(\mathfrak{m}_{\mathbf{C}}\right)$ is standard in $\phi\left(\left(\mathfrak{f}_{1}\right)_{\mathbf{C}}\right)$, i.e.

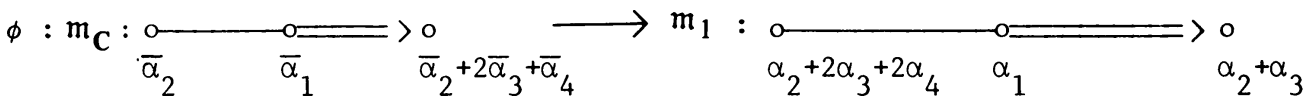

It is clear that $\phi$ must be defined in the following way:

$$
\begin{array}{ll}
\phi\left(\varepsilon_{1}\right)=\frac{1}{2}\left(\varepsilon_{1}+\varepsilon_{2}+\varepsilon_{3}-\varepsilon_{4}\right), & \phi\left(\varepsilon_{2}\right)=\frac{1}{2}\left(\varepsilon_{1}+\varepsilon_{2}-\varepsilon_{3}+\varepsilon_{4}\right), \\
\phi\left(\varepsilon_{3}\right)=\frac{1}{2}\left(\varepsilon_{1}-\varepsilon_{2}+\varepsilon_{3}+\varepsilon_{4}\right), & \phi\left(\varepsilon_{4}\right)=\frac{1}{2}\left(-\varepsilon_{1}+\varepsilon_{2}+\varepsilon_{3}+\varepsilon_{4}\right) .
\end{array}
$$

We have

$$
\begin{aligned}
D_{K} & =\left\{\lambda=\sum_{i=1}^{4} a_{i} \varepsilon_{i} ; a_{1} \geqslant \cdots \geqslant a_{4} \geqslant 0,2 a_{i} \in \mathbf{Z}, a_{i}-a_{j} \in \mathbf{Z}, 1<i, j<4\right\} . \\
D_{K_{1}} & =\left\{\mu=\sum_{i=1}^{4} b_{i} \varepsilon_{i} ; b_{1} \geqslant b_{2} \geqslant b_{3} \geqslant\left|b_{4}\right|, b_{i}-b_{j} \in \mathbf{Z}, 2 b_{i} \in \mathbf{Z}, i=1, \ldots, 4\right\} . \\
D_{\phi(M)} & =\left\{\gamma=\sum_{i=1}^{3} q_{i} \varepsilon_{i} ; q_{1} \geqslant q_{2} \geqslant q_{3} \geqslant 0,2 q_{i} \in \mathbf{Z}, q_{i}-q_{j} \in \mathbf{Z}\right\} .
\end{aligned}
$$

We use the vector notation $\left(a_{1}, \ldots, a_{n}\right)=\sum_{1}^{n} a_{i} \varepsilon_{i}$ for $a_{i} \in \mathbf{C}$. 
THEOREM 10.9. (Notation as in 10.8.) Let $\lambda=\sum_{i=1}^{4} a_{i} \varepsilon_{i} \in D_{K}$; then

$$
\pi_{\left.\lambda\right|_{M}}=\sum_{\substack{\left.q_{1}^{\prime}, \ldots, q_{4}^{\prime}\right)=\phi\left(q_{1}, \ldots, q_{4}\right) \\\left(q_{1}, \ldots, q_{4}\right) \in D_{K_{1}} \\ a_{1}>q_{1}>\cdots>a_{4}>\left|q_{4}\right|}} \sum_{\substack{\gamma=\left(b_{1}, b_{2}, b_{3}\right) \in D_{\phi(M)} \\ q_{1}^{\prime}>b>\cdots>q_{3}^{\prime}>b_{3}>\left|q_{4}^{\prime}\right| \\ a_{i}-q_{j} \in \mathbf{Z}}} \pi_{\gamma} \circ \phi .
$$

\section{Classification of positive systems of compact roots.}

11.1. We are now in a position to use the general results of $\$ \S 1-9$ in order to solve our problem. As a consequence of Theorem 8.5 and Remark 8.6 we have seen that we only need to consider discrete series representations with trivial infinitesimal character. The first step will be to give a complete parametrizations of such discrete series. As second step we find the $M$-types of the minimal $K$-types of these discrete series. In view of Theorem 5.1 this will determine the $M$-parameter of the principal series where such discrete series may occur. The equation of the infinitesimal character (Lemma 7.1(1)) will exclude some of these possibilities. Finally we will use Corollary 9.3 and Lemma 16.6 to solve the problem in most cases.

For the remaining cases we will have to use other $K$-type arguments to get the results (see $\$ 17)$. The whole of these results are contained in the tables at the end.

We retain the notation of $\S 10$. If $\beta \in \Delta$, we denote by $s_{\beta}$ the reflection by $\beta$. The following result is an immediate consequence of [3].

LEMMA 11.2. Let $P_{1}, \ldots, P_{n}$ be all the distinct systems of positive roots for $\Delta$ containing $\Delta_{k}^{+}$(i.e. $\left.P_{i} \cap \Delta_{k}=\Delta_{k}^{+}\right)$. Let

$$
\left\{\pi_{\delta_{p_{i}}}\right\}_{i=1}^{n}
$$

be the corresponding discrete series. Then if $\omega$ is a discrete series with trivial infinitesimal character, $\omega$ is equivalent to $\pi_{\delta_{P_{i}}}$ for some $i$.

In view of the previous lemma a complete parametrization of the discrete series with trivial infinitesimal character is given, once we know all the systems $\boldsymbol{P}_{i}$ 's.

We will exhibit them by a case by case argument. First we need the following well-known result:

Lemma 11.3. Let $A=\left\{P \subset \Delta, P\right.$ positive for $\Delta$ so that $\left.P \cap \Delta_{k}=\Delta_{k}^{+}\right\}$. Then $|A|=|W| /\left|W_{k}\right|(||=$ cardinality $)$.

11.4. $G=\operatorname{Spin}(2 n, 1)$. Let $B_{1}=\Delta^{+}$and define $B_{2}=s_{\varepsilon_{n}} B_{1}$.

LEMMA 11.5. The systems of positive roots containing $\Delta_{k}^{+}$are precisely $B_{1}$ and $B_{2}$.

Proof. $B_{1}$ and $B_{2}$ are distinct. The simple roots of $B_{2}$ are $\alpha_{1}, \ldots, \alpha_{n-2}$, $\varepsilon_{n-1}+\varepsilon_{n},-\varepsilon_{n}$. Since $\alpha_{n-1}=\varepsilon_{n-1}+\varepsilon_{n}+2\left(-\varepsilon_{n}\right)$ is in $B_{2}$, it is clear that $\Delta_{k}^{+} \subset B_{2}$. On the other hand, there are exactly two such systems, thus the lemma.

11.6. $G=\operatorname{SU}(n, 1)$. We also use the notation $\Delta^{+}: \varepsilon_{1} \geqslant \cdots>\varepsilon_{n+1}$ for the fundamental Weyl chamber associated to $\Delta^{+}$. We define $A_{i}, 0<i<n$, a system of positive roots for $\Delta$, as follows:

$$
A_{n}=\Delta^{+} \text {and } \gamma_{j}^{n}=\alpha_{j}, \quad j=1, \ldots, n .
$$


Suppose $A_{i}$ and $\gamma_{j}^{i}$ have been defined. Then if $0 \leqslant i-1$, define $A_{i-1}=s_{\gamma_{i}} A_{i}$ and $\gamma_{j}^{i-1}=s_{\gamma_{i}} \gamma_{j}^{i}$.

LEMMA 11.7. (1) The systems $A_{i}$ are all distinct.

(2) If $Q$ is positive and contains $\Delta_{k}^{+}$, then $Q=A_{i}$ for some $i$.

PRoOF. (1'). $\gamma_{i}^{i}=\alpha_{i}+\cdots+\alpha_{n}, 1 \leqslant i \leqslant n$.

Suppose $\gamma_{k}^{k}=\alpha_{k}+\cdots+\alpha_{n}$ for $i \leqslant k \leqslant n$, then $\gamma_{i-1}^{i-1}=s_{\gamma_{i}} \gamma_{i-1}^{i}=$ $s_{\gamma_{i}}^{i} \gamma_{i+1}^{i+1} \cdots s_{\gamma_{n}^{n}} \gamma_{i-1}^{n}=s_{\gamma_{i}^{i}} \alpha_{i-1}=\alpha_{i-1}+\cdots+\alpha_{n}$. Since $\gamma_{n}^{n}=\alpha_{n}$, the induction argument applies and $\left(1^{\prime}\right)$ is true.

(2') $A_{i}: \varepsilon_{1} \geqslant \cdots \geqslant \varepsilon_{i} \geqslant \varepsilon_{n+1} \geqslant \varepsilon_{i+1} \geqslant \cdots \geqslant \varepsilon_{n}$, that is

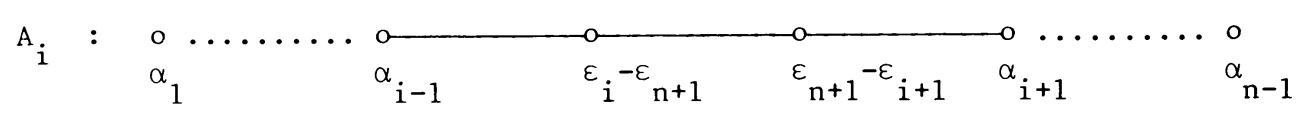

For $i=n,\left(2^{\prime}\right)$ is true. Suppose we have proved it for $i \leqslant k \leqslant n$, then by $\left(1^{\prime}\right)$ $A_{i-1}=s_{\varepsilon_{i}-\varepsilon_{n+1}} A_{i}$. But $s_{\varepsilon_{i}-\varepsilon_{n+1}}$ interchanges $\varepsilon_{i}$ and $\varepsilon_{n+1}$, hence (2') follows. Lemma 11.3 says that there are exactly $(n+1) ! / n !=(n+1)$ systems of positive roots containing $\Delta_{k}^{+}$; this, together with $\left(2^{\prime}\right)$ gives the result.

11.8. $G=\operatorname{Sp}(n, 1)$. For $0 \leqslant i \leqslant n$, we define $n+1$ systems of positive roots as follows:

$$
C_{n}=\Delta^{+} \quad \text { and } \quad \gamma_{j}^{n}=\alpha_{j}, \quad i=1, \ldots, n+1 .
$$

Suppose $C_{i}$ has been defined with simple roots $\gamma_{j}^{i}$, then if $i-1>0$, define

$$
C_{i-1}=s_{\gamma_{i}^{i}} C_{i} \text { and } \gamma_{j}^{i-1}=s_{\gamma_{i}} \gamma_{j}^{i}
$$

LEMMA 11.9. The $C_{i}$ 's are all distinct and they exhaust all the systems of positive roots containing $\Delta_{k}^{+}$.

PROOF. (1) $C_{i}: \varepsilon_{1} \geqslant \cdots \geqslant \varepsilon_{i} \geqslant \varepsilon_{n+1} \geqslant \varepsilon_{i+1} \geqslant \cdots \geqslant \varepsilon_{n} \geqslant 0$; that is

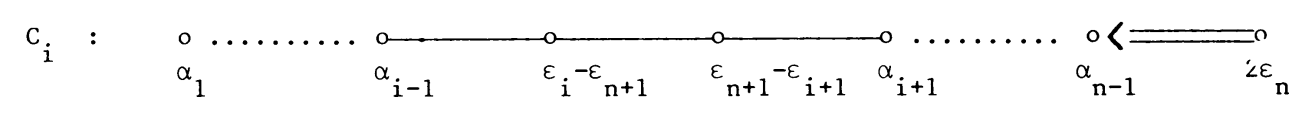

As in Lemma 2.2, $\gamma_{i}^{i}=\alpha_{i}+\cdots+\alpha_{n}, 1<i \leqslant n$; thus (1). Clearly, the $C_{i}$ 's are all distinct, and $C_{i}$ contains $\Delta_{k}^{+}: \varepsilon_{1} \geqslant \cdots \geqslant \varepsilon_{n} \geqslant 0, \varepsilon_{n+1} \geqslant 0$. Since there are $(n+1)$ positive systems containing $\Delta_{k}^{+}$, the lemma follows.

11.10. $G=F_{4}$. Define

$$
F_{2}=s_{\alpha_{4}} F_{3}: \quad \begin{array}{llll}
\alpha_{1} & \alpha_{3}=\Delta^{+}, & & \\
F_{1}=s_{\alpha_{3}+\alpha_{4}} F_{2}: & \alpha_{2} & \alpha_{3}+\alpha_{4} & -\alpha_{4} \\
\alpha_{1} & & \alpha_{2}+2 \alpha_{3}+2 \alpha_{4}-\alpha_{3}-\alpha_{4}
\end{array}
$$

Thus it is clear:

LEMMA 11.11. The systems of positive roots containing $\Delta_{k}^{+}$are $F_{1}, F_{2}, F_{3}$. 
11.12. We now compute the $2 \delta_{n}$ 's relative to the particular systems of positive roots just introduced. We use the convention $\Sigma_{a}^{b}=0$ if $b<a, a, b \in \mathbf{Z}$.

11.13. $\operatorname{Spin}(2 n, 1)$.

LEMMA 11.14. $2 \delta_{n}^{B_{1}}=\sum_{j=1}^{n} \varepsilon_{j}$ and $2 \delta_{n}^{B_{2}}=\sum_{j=1}^{n-1} \varepsilon_{j}-\varepsilon_{n}$.

Proof. Following Bourbaki [2]:

$$
\begin{aligned}
2 \delta_{k} & =\sum_{j=1}^{n-1} 2(n-j) \varepsilon_{j}, \\
2 \delta_{B_{1}} & =\sum_{j=1}^{n}(2 n-2 j+1) \varepsilon_{j}, \quad \text { and } \\
2 \delta_{B_{2}} & =s_{\varepsilon_{n}} 2 \delta_{B_{1}}=\sum_{j=1}^{n-1}(2 n-2 j+1) \varepsilon_{j}-\varepsilon_{n} .
\end{aligned}
$$

The identities then follow.

11.15. SU $(n, 1)$.

LEMMA 11.16. $2 \delta_{n}^{A_{i}}=\sum_{j=1}^{i} \varepsilon_{j}-\sum_{j=i+1}^{n} \varepsilon_{j}+(n-2 i) \varepsilon_{n+1}, 0 \leqslant i \leqslant n$.

Proof. Following Bourbaki [2]:

$$
\begin{aligned}
2 \delta_{k} & =2 \delta_{k}^{\Delta_{k}^{+}}=\sum_{j=1}^{n}(n-1-2 j+2) \varepsilon_{j}=\sum_{j=1}^{n}(n-2 j+1) \varepsilon_{j} \\
2 \delta_{A^{i}} & =\sum_{j=1}^{i}(n-2 j+2) \varepsilon_{j}+\sum_{j=i+1}^{n}(n-2 j) \varepsilon_{j}+(n-2 i) \varepsilon_{n+1}
\end{aligned}
$$

for $0 \leqslant i \leqslant n$.

Suppose (1) is true for $i+1$, then

$$
\begin{aligned}
2 \delta_{A^{i}}= & s_{\varepsilon_{i+1}-\varepsilon_{n+1}} 2 \delta_{A_{i+1}} \\
= & s_{\varepsilon_{i+1}-\varepsilon_{n+1}}\left(\sum_{j=1}^{i+1}(n-2 j+2) \varepsilon_{j}+\sum_{j=i+2}^{n}(n-2 j) \varepsilon_{j}+(n-2(i+1)) \varepsilon_{n+1}\right) \\
= & \sum_{j=1}^{i}(n-2 j+2) \varepsilon_{j}+(n-2(i+1)+2) \varepsilon_{n+1} \\
& +\sum_{j=i+2}^{n}(n-2 j) \varepsilon_{j}+(n-2(i+1)) \varepsilon_{i+1} \\
= & \sum_{j=1}^{i}(n-2 j+2) \varepsilon_{j}+\sum_{j=i+1}^{n}(n-2 j) \varepsilon_{j}+(n-2 i) \varepsilon_{n+1} .
\end{aligned}
$$

Since the formula is true for $i=n$, the induction argument applies to prove (1). 
Hence

$$
\begin{aligned}
2 \delta_{n}^{A_{i}}= & 2 \delta_{A_{i}}-2 \delta_{k}=\sum_{j=1}^{i}(n-2 j+2) \varepsilon_{j} \\
& +\sum_{j=i+1}^{n}(n-2 j) \varepsilon_{j}+(n-2 i) \varepsilon_{n+1}-\sum_{j=1}^{n}(n-2 j+1) \varepsilon_{j} \\
= & \sum_{j=1}^{i} \varepsilon_{j}-\sum_{j=i+1}^{n} \varepsilon_{j}+(n-2 i) \varepsilon_{n+1} .
\end{aligned}
$$

11.17. $\mathrm{Sp}(n, 1)$.

LEMMA 11.18. $2 \delta_{n}^{C_{i}}=\sum_{j=1}^{i} 2 \varepsilon_{j}+2(n-i) \varepsilon_{n+1}$ for $i=0, \ldots, n$.

Proof. In this case

$$
\begin{gathered}
2 \delta_{k}=2 \delta_{k}^{\Delta_{k}^{+}}=\sum_{j=1}^{n} 2(n-j+1) \varepsilon_{j}+2 \varepsilon_{n+1} \\
2 \delta_{C_{i}}=\sum_{j=1}^{i} 2(n+1-j+1) \varepsilon_{j}+\sum_{j=i+1}^{n} 2(n+1-j) \varepsilon_{j}+2(n+1-i) \varepsilon_{n+1}
\end{gathered}
$$

for $0 \leqslant i \leqslant n$.

Suppose (1) is true for $i+1$, then

$$
\begin{aligned}
2 \delta_{C_{i}}= & s_{\varepsilon_{i+1}-\varepsilon_{n+1}} 2 \delta_{C_{i+1}} \\
= & s_{\varepsilon_{i+1}-\varepsilon_{n+1}}\left(\sum_{j=1}^{i+1} 2(n+1-j+1) \varepsilon_{j}+\sum_{j=i+2}^{n} 2(n+1-j) \varepsilon_{j}+2(n-i) \varepsilon_{n+1}\right) \\
= & \sum_{j=1}^{i} 2(n+1-j+1) \varepsilon_{j}+2(n+1-i) \varepsilon_{n+1} \\
& \quad+\sum_{j=i+2}^{n} 2(n+1-j) \varepsilon_{j}+2(n-i) \varepsilon_{i+1} \\
= & \sum_{j=1}^{i} 2(n+1-j+1) \varepsilon_{j}+\sum_{j=i+1}^{n} 2(n+1-j) \varepsilon_{j}+2(n+1-i) \varepsilon_{n+1} .
\end{aligned}
$$

Since the formula is true for $i=n$, apply induction to get (1). Thus

$$
2 \delta_{n}^{C_{i}}=2 \delta_{C_{i}}-2 \delta_{k}=\sum_{j=1}^{i} 2 \varepsilon_{j}+2(n-i) \varepsilon_{n+1} \text {. }
$$

11.19. $F_{4}$

LEMMA 11.20. $2 \delta_{n}^{F_{1}}=2 \varepsilon_{1}+2 \varepsilon_{2}+2 \varepsilon_{3}, 2 \delta_{n}^{F_{2}}=3 \varepsilon_{1}+\varepsilon_{2}+\varepsilon_{3}+\varepsilon_{4}, 2 \delta_{n}^{F_{3}}=4 \varepsilon_{1}$.

Proof. $2 \delta_{k}=\sum_{j=1}^{4}(2 \cdot 4-2 j+1) \varepsilon_{j}=7 \varepsilon_{1}+5 \varepsilon_{2}+3 \varepsilon_{3}+\varepsilon_{4}$ and following [2]:

$$
\begin{aligned}
2 \delta_{F_{1}} & =16 \alpha_{1}+30\left(\alpha_{2}+2 \alpha_{3}+2 \alpha_{4}\right)-42\left(\alpha_{3}+\alpha_{4}\right)+22 \alpha_{3} \\
& =16 \alpha_{1}+30 \alpha_{2}+40 \alpha_{3}+18 \alpha_{4} \\
& =16\left(\varepsilon_{2}-\varepsilon_{3}\right)+30\left(\varepsilon_{3}-\varepsilon_{4}\right)+40 \varepsilon_{4}+9\left(\varepsilon_{1}-\varepsilon_{2}-\varepsilon_{3}-\varepsilon_{4}\right) \\
& =9 \varepsilon_{1}+7 \varepsilon_{2}+5 \varepsilon_{3}+\varepsilon_{4},
\end{aligned}
$$




$$
\begin{aligned}
2 \delta_{F_{2}} & =16 \alpha_{1}+30 \alpha_{2}+42\left(\alpha_{3}+\alpha_{4}\right)-22 \alpha_{4}=16 \alpha_{1}+30 \alpha_{2}+42 \alpha_{3}+20 \alpha_{4} \\
& =16\left(\varepsilon_{2}-\varepsilon_{3}\right)+30\left(\varepsilon_{3}-\varepsilon_{4}\right)+42 \varepsilon_{4}+10\left(\varepsilon_{1}-\varepsilon_{2}-\varepsilon_{3}-\varepsilon_{4}\right) \\
& =10 \varepsilon_{1}+6 \varepsilon_{2}+4 \varepsilon_{3}+2 \varepsilon_{4}, \\
2 \delta_{F_{3}} & =11 \varepsilon_{1}+5 \varepsilon_{2}+3 \varepsilon_{3}+\varepsilon_{4} .
\end{aligned}
$$

The result follows.

12. Computation of $M$-types for $\tau_{2 \delta_{n}}$.

12.1. If $P$ is a system of positive roots for $\Delta$ containing $\Delta_{k}^{+}$, let us denote by $\tau_{2 \delta_{n}^{p}}$ the $K$-module of highest weight $2 \delta_{n}^{P}$. Then $\tau_{2 \delta_{n}^{P}}$ is the minimal $K$-type of the discrete series $\pi_{\delta_{p}}([15],[19])$. We are now ready to compute the $M$-types for the minimal $K$-types of the discrete series we are studying.

TheOREM 12.2. Let $G=\operatorname{Spin}(2 n, 1), n \geqslant 2 . \tau_{2 \delta_{n}^{B_{1}}}$ and $\tau_{2 \delta_{n}^{B_{2}}}$ are both irreducible as $M$-representations and they are equivalent to $\pi_{\mu_{1}}$, where $\mu_{1}=\sum_{i=1}^{n-1} \varepsilon_{i}$.

Proof. $2 \delta_{n}^{B_{1}}=\sum_{j=1}^{n} \varepsilon_{i}$. We need to find all the $(n-1)$-tuples $\left(b_{1}, \ldots, b_{n-1}\right) \in$ $\mathbf{Z}^{n-1}$ satisfying:

(i) $b_{i}-b_{j} \in \mathbf{Z}, 2 b_{i} \in \mathbf{Z}$ and $b_{1} \geqslant \cdots \geqslant b_{n-1} \geqslant 0$,

(ii) $a_{1} \geqslant b_{1} \geqslant \cdots \geqslant b_{n-1} \geqslant\left|a_{n}\right|$,

(iii) $a_{i}-b_{j} \in \mathbf{Z}$,

where $a_{1}=\cdots=a_{n}=1$.

If $\left(b_{1}, \ldots, b_{n-1}\right) \in \mathbf{Z}^{n-1}$ and satisfies (ii), then $b_{1}=\cdots=b_{n-1}=1$ and hence all the other conditions are, a fortiori, satisfied. The result follows from Theorem 10.3. The same proof applies to $2 \delta_{n}^{B_{2}}$ since the absolute value of the coefficient of $\varepsilon_{n}$ is still 1 .

THEOREM 12.3. Let $G=\operatorname{SU}(n, 1), n \geqslant 2$. The M-types of $\tau_{2 \delta_{n}^{A_{i}}}(0 \leqslant i<n)$ are $\pi_{\mu_{i} \text {, }}$, $0 \leqslant i \leqslant n-1, \pi_{\mu_{2}^{i}} 1 \leqslant i \leqslant n-1$, and $\pi_{\mu_{3}^{i}} 1 \leqslant i \leqslant n$ where

$$
\begin{gathered}
\mu_{1}^{i}=\frac{n-2 i-1}{2}\left(\varepsilon_{1}+\varepsilon_{n+1}\right)+\sum_{j=2}^{i+1} \varepsilon_{j}-\sum_{j=i+2}^{n} \varepsilon_{j}, \quad 0 \leqslant i \leqslant n-1, \\
\mu_{2}^{i}=\frac{n-2 i}{2}\left(\varepsilon_{1}+\varepsilon_{n+1}\right)+\sum_{j=2}^{i} \varepsilon_{j}-\sum_{j=i+2}^{n} \varepsilon_{j}, \quad 1 \leqslant i \leqslant n-1, \\
\mu_{3}^{i}=\frac{n-2 i+1}{2}\left(\varepsilon_{1}+\varepsilon_{n+1}\right)+\sum_{j=2}^{i} \varepsilon_{j}-\sum_{j=i+1}^{n} \varepsilon_{j}, \quad 1 \leqslant i \leqslant n .
\end{gathered}
$$

The multiplicity of each M-type is one.

Proof. We have proved that

$$
2 \delta_{n}^{A_{i}}=\sum_{j=1}^{i} \varepsilon_{j}-\sum_{j=i+1}^{n} \varepsilon_{j}+(n-2 i) \varepsilon_{n+1}, \quad 0<i<n .
$$

We handle the following cases $i=0,1 \leqslant i \leqslant n-1, i=n$, one at a time. If $1 \leqslant i \leqslant n-1$, set $a_{1}=\cdots=a_{i}=1, a_{i+1}=\cdots=a_{n}=-1, a_{n+1}=(n-2 i)$. 
We need to find all the $n$-tuples $\left(c_{1}, \ldots, c_{n}\right) \in \mathbf{Z}^{n}$ satisfying:

(i) $a_{1} \geqslant c_{2} \geqslant \cdots \geqslant a_{n-1} \geqslant c_{n} \geqslant a_{n}$ and

(ii) $\sum_{i=1}^{n} a_{i}=\sum_{i=1}^{n} c_{i}$.

Let $c=\left(c_{1}, \ldots, c_{n}\right)$ be such an $n$-tuple. Then it is either

$$
\begin{gathered}
c_{1}=-1, \quad c_{2}=\cdots=c_{i}=1, \quad c_{i+1}=1, \quad c_{i+2}=\cdots=c_{n}=-1, \\
c_{1}=0, \quad c_{2}=\cdots=c_{i}=1, \quad c_{i+1}=0, \quad c_{i+2}=\cdots=c_{n}=-1, \\
c_{1}=1, \quad c_{2}=\cdots=c_{i}=1, \quad c_{i+1}=-1, \quad c_{i+2}=\cdots=c_{n}=-1 .
\end{gathered}
$$

Indeed because the $c_{i}$ 's are integers, (i) forces $c_{2}=\cdots=c_{i}=1, c_{i+2}=\cdots=c_{n}$ $=-1$ and $c_{i+1}=-1,0,1$. Apply (ii) to get the values for $c_{1}$.

In view of Theorem 10.5, the result is clear if $1 \leqslant i \leqslant n-1$. In the case $i=0$ or $n$, the proof is similar and we omit it.

THEOREM 12.4. Let $G=\operatorname{Sp}(n, 1), n \geqslant 2$. The M-types of $\tau_{2 \delta_{n}}, 0 \leqslant i \leqslant n$, are the ones parametrized by the following highest weights:

$$
\begin{aligned}
& \mu_{1}^{i}=(n-i)\left(\varepsilon_{1}+\varepsilon_{n+1}\right)+2 \sum_{j=2}^{i-1} \varepsilon_{j}, \quad 2 \leqslant i \leqslant n-1, i=0, n, \\
& \mu_{2}^{i}=\left(n-i-\frac{1}{2}\right)\left(\varepsilon_{1}+\varepsilon_{n+1}\right)+2 \sum_{j=2}^{i-1} \varepsilon_{j}+\varepsilon_{i}, \quad 2 \leqslant i \leqslant n-1, \\
& \mu_{3}^{i}=\left(n-i+\frac{1}{2}\right)\left(\varepsilon_{1}+\varepsilon_{n+1}\right)+2 \sum_{j=2}^{i-1} \varepsilon_{j}+\varepsilon_{i}, \quad 2 \leqslant i \leqslant n-1, i=n, \\
& \mu_{4}^{i}=(n-i)\left(\varepsilon_{1}+\varepsilon_{n+1}\right)+2 \sum_{j=2}^{i-1} \varepsilon_{j}+\varepsilon_{i}+\varepsilon_{i+1}, \quad 2 \leqslant i \leqslant n-1, \\
& \mu_{5}^{i}=(n-i+1)\left(\varepsilon_{1}+\varepsilon_{n+1}\right)+2 \sum_{j=2}^{i-1} \varepsilon_{j}+2 \varepsilon_{i}, \quad 2 \leqslant i \leqslant n-1, i=n, i=1, \\
& \mu_{6}^{i}=(n-i)\left(\varepsilon_{1}+\varepsilon_{n+1}\right)+2 \sum_{j=2}^{i-1} \varepsilon_{j}+2 \varepsilon_{i}, \quad 2 \leqslant i \leqslant n-1, i=1, \\
& \mu_{7}^{i}=(n-i-1)\left(\varepsilon_{1}+\varepsilon_{n+1}\right)+2 \sum_{j=2}^{i-1} \varepsilon_{j}+2 \varepsilon_{i}, \quad 2 \leqslant i \leqslant n-1, i=1, \\
& \mu_{8}^{i}=\left(n-i+\frac{1}{2}\right)\left(\varepsilon_{1}+\varepsilon_{n+1}\right)+2 \sum_{j=2}^{i-1} \varepsilon_{j}+2 \varepsilon_{i}+\varepsilon_{i+1}, \quad 2 \leqslant i \leqslant n-1, i=1, \\
& \mu_{10}^{i}=(n-i)\left(\varepsilon_{1}+\varepsilon_{n+1}\right)+2 \sum_{j=2}^{i-1} \varepsilon_{j}+2 \varepsilon_{i}+2 \varepsilon_{i+1}, \quad 2 \leqslant i \leqslant n-1, i=1, \\
& =\left(n-i-\frac{1}{2}\right)\left(\varepsilon_{1}+\varepsilon_{n+1}\right)+2 \sum_{j=2}^{i-1} \varepsilon_{j}+2 \varepsilon_{i}+\varepsilon_{i+1}, \quad 2 \leqslant i \leqslant n-1, i=1,
\end{aligned}
$$

where $\sum_{j=2}^{i-1} \varepsilon_{j}=0$ if $i \leqslant 2$ and $\sum_{j=2}^{i} \varepsilon_{j}=0$ if $i=1$. The multiplicity of each $M$-type is one. 
Proof. $2 \delta_{n}^{C_{i}}=\sum_{j=1}^{i} 2 \varepsilon_{j}+2(n-i) \varepsilon_{n+1}$ for $i=0, \ldots, n$.

Let $a=\left(a_{1}, \ldots, a_{n+1}\right)$ be defined by $\sum_{i=1}^{n+1} a_{i} \varepsilon_{i}=2 \delta_{n}^{C_{i}}$. We want to find all the $n$-tuple in $\mathbf{Z}^{n}, b=\left(b_{1}, \ldots, b_{n}\right)$ satisfying:

(i) $b_{1} \geqslant 0, b_{2} \geqslant \cdots \geqslant b_{n} \geqslant 0$,

(ii) $b_{j} \geqslant a_{j+1}, 2 \leqslant j \leqslant n-1$,

(iii) $a_{j} \geqslant b_{j+1}, 1 \leqslant j \leqslant n-1$,

(iv) $\sum_{j=1}^{n}\left(a_{j}+b_{j}\right) \in 2 \mathbf{Z}$,

(v)

$$
\begin{aligned}
\tilde{m}_{\lambda}\left(\mu_{b}\right) & \equiv \tilde{m}_{\lambda}(b) \\
& =\sum_{L \subset\{1, \ldots, n\}}(-1)^{|L|}\left(\begin{array}{c}
n-2-|L|+\frac{1}{2}\left(-b_{1}+\sum_{i=1}^{n} A_{i}\right)-\sum_{i \in L} A_{i} \\
n-2
\end{array}\right) \neq 0
\end{aligned}
$$

$\left(\mu_{b}=\sum_{i=1}^{n} b_{i} \varepsilon_{i}\right)$.

If $b$ is such an $n$-tuple set $b_{0}^{j}=\left(a_{n+1}+b_{1}-2 j\right) / 2$ for $j=0, \ldots, \min \left(a_{n+1}, b_{1}\right)$. Theorem 10.7 says that $\mu_{j}=\left(b_{0}^{j}, b_{2}, \ldots, b_{n}\right) \equiv \sum_{i=2}^{n} b_{j} \varepsilon_{i}+b_{0}^{j}\left(\varepsilon_{1}+\varepsilon_{n+1}\right)$ give all the $M$-types of $\tau_{2 \delta_{n}}$, when $b$ varies through all the possible $n$-tuples of that kind.

We need to look at the following cases:

(1) $i=0$,

(2) $i=1$,

(3) $2 \leqslant i \leqslant n-1$,

(4) $i=n$.

(1) If $i=0$, then $a_{1}=\cdots=a_{n}=0$ and $a_{n+1}=2 n$. The $\mathbf{Z} n$-tuples satisfying (i)-(iv) are of the form $b_{k}=(2 k, 0, \ldots, 0)$ for $k \in \mathbf{Z}_{+}$. Since $A_{1}=\cdots=A_{n}=$ 0 , then $\tilde{m}_{\lambda}\left(\mu_{b_{k}}\right) \neq 0$ implies $k=0$; therefore the only $n$-tuple in $\mathbf{Z}^{n}$ for which (i)-(v) is true is $(0, \ldots, 0)$, with multiplicity one. The only choice for $b_{0}^{j}$ is $b_{0}^{0}=2 n / 2=n$. Hence the theorem is true in this case, with the usual convention that $\sum_{j=2}^{i-1}=0$ if $i-1<2$.

(2) If $i=1$, then $a_{1}=2, a_{2}=\cdots=a_{n}=0$ and $a_{n+1}=2(n-1)$. Let $b=$ $\left(b_{1}, \ldots, b_{n}\right) \in \mathbf{Z}^{n}$, satisfying (i)-(iii). Then $b_{3}=\cdots=b_{n}=0$ (if $n \geqslant 3$ ) and $0 \leqslant b_{2} \leqslant 2, b_{1} \geqslant 0$. $\sum_{i=1}^{n} a_{i}+b_{i} \in 2 \mathrm{Z}$ forces $b_{1}+b_{2} \in 2 \mathrm{Z}$; consequently the possible $n$-tuples in $Z^{n}$ for which (i)-(iv) is true are of the form:

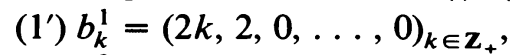

$\left(2^{\prime}\right) b_{k}^{2}=(2 k+1,1,0, \ldots, 0)_{k \in \mathbf{Z}_{+}}$,

(3') $b_{k}^{3}=(2 k, 0, \ldots, 0)_{k \in \mathbf{z}_{+}}$.

The only $n$-tuple in $Z^{n}$ of the form (1') satisfying (v) is $(0,2,0, \ldots, 0)$ with $\tilde{m}_{\lambda}\left(b_{0}^{1}\right)=1$.

Indeed $A_{1}=\cdots=A_{n}=0$ and

$$
\tilde{m}_{\lambda}\left(b_{k}^{1}\right)=\sum_{L \subset\{1, \ldots, n\}}(-1)^{|L|}\left(\begin{array}{c}
n-2-|L|-k \\
n-2
\end{array}\right) \neq 0 \Rightarrow k=0
$$

and $|L|=0$. Therefore $\tilde{m}_{\lambda}\left(b_{k}^{1}\right)=0$ if $k \neq 0$ and $\tilde{m}_{\lambda}\left(b_{k}^{1}\right)=1$ if $k=0$.

The only $n$-tuple in $Z^{n}$ of the form $\left(2^{\prime}\right)$ satisfying $(v)$ is $(1,1,0, \ldots, 0)$ with $\tilde{m}_{\lambda}\left(b_{0}^{2}\right)=1$. Indeed $A_{1}=1, A_{2}=\cdots=A_{n}=0$ and 


$$
\begin{aligned}
\tilde{m}_{\lambda}\left(b_{k}^{2}\right)= & \sum_{1 \in L}(-1)^{|L|}\left(\begin{array}{c}
n-2-L-k-1 \\
n-2
\end{array}\right) \\
& +\sum_{1 \notin L}(-1)^{|L|}\left(\begin{array}{c}
n-2-|L|-k \\
n-2
\end{array}\right) \\
= & \sum_{1 \notin L}(-1)^{|L|}\left(\begin{array}{c}
n-2-|L|-k \\
n-2
\end{array}\right) .
\end{aligned}
$$

Hence if $\tilde{m}_{\lambda}\left(b_{k}^{2}\right) \neq 0$, then $k=0$ and in particular $\tilde{m}_{\lambda}\left(b_{0}^{2}\right)=1$.

The only $n$-tuple in $Z^{n}$ of the form (3') satisfying (v) is $(2,0, \ldots, 0)$ with $\tilde{m}_{\lambda}\left(b_{1}^{3}\right)=1$. In fact $A_{1}=2, A_{2}=\cdots=A_{n}=0$,

$$
\begin{aligned}
\tilde{m}_{\lambda}\left(b_{k}^{3}\right)= & \sum_{2 \in L}(-1)^{|L|}\left(\begin{array}{c}
n-2-|L|+\frac{1}{2}(-2 k+2)-2 \\
n-2
\end{array}\right) \\
& +\sum_{2 \notin L}(-1)^{|L|}\left(\begin{array}{c}
n-2-|L|-k+1 \\
n-2
\end{array}\right) \\
= & \sum_{2 \notin L}(-1)^{|L|}\left(\begin{array}{c}
n-2-|L|-k+1 \\
n-2
\end{array}\right) .
\end{aligned}
$$

If $k \geqslant 2$, it is clear that $\tilde{m}_{\lambda}\left(b_{k}^{3}\right)=0$.

If $k=0$,

$$
\begin{aligned}
\tilde{m}_{\lambda}\left(b_{0}^{3}\right) & =\sum_{\substack{2 \notin L \\
|L|=0,1}}(-1)^{|L|}\left(\begin{array}{c}
n-2-|L|+1 \\
n-2
\end{array}\right) \\
& =\left(\begin{array}{c}
n-2+1 \\
n-2
\end{array}\right)-(n-1)\left(\begin{array}{l}
n-2 \\
n-2
\end{array}\right)=0 .
\end{aligned}
$$

If $k=1, \tilde{m}_{\lambda}\left(b_{1}^{3}\right)=1$. Concluding, the $n$-tuples in $Z^{n}$ satisfying (i)-(v) are $(0,2, \ldots, 0),(1,1,0, \ldots, 0)$ and $(2,0, \ldots, 0)$.

Set as usual $b_{0}^{j}=\left(a_{n+1}+b_{1}-2_{j}\right) / 2$ for $j=0, \ldots, \min \left(a_{n+1}, b_{1}\right)$ and $b_{1}=$ $0,1,2$.

If $b_{1}=0$, then $j=0$ and $b_{0}^{0}=(n-1)$.

If $b_{1}=1$, then $j=0,1$ and $b_{0}^{0}=(n-1)+\frac{1}{2}, b_{0}^{1}=(n-1)-\frac{1}{2}$.

If $b_{1}=2$, then $j=0,1,2$ and $b_{0}^{0}=n, b_{0}^{1}=n-1, b_{0}^{2}=n-2$.

Hence, the $M$-types are the ones described.

(3) $2 \leqslant i \leqslant n-1$. In this case $a_{1}=\cdots=a_{i}=2$ and $a_{i+1}=\cdots=a_{n}=0$ and $a_{n+1}=2(n-i)$.

Let $b=\left(b_{1}, \ldots, b_{n}\right)$ be a $\mathbf{Z} n$-tuple satisfying (i)-(iii). Then $b_{2}=\cdots=b_{i-1}=$ 2 (if $i \geqslant 3$ ), $0 \leqslant b_{i}, \quad b_{i+1} \leqslant 2, b_{i} \geqslant b_{i+1}$, and $b_{i+2}=\cdots=b_{n}=0, b_{1}>0$. $\sum_{i=1}^{n}\left(a_{i}+b_{i}\right) \in 2 Z$ forces $b_{1}+b_{i+1} \in 2 Z$; it is now clear that the possible $n$-tuples in $Z^{n}$ satisfying (i)-(iv) are 


$\begin{array}{llllll}\left(1^{\prime \prime}\right) & (2 k, 2, \ldots, 2, & 0, & 0, & 0, \ldots, 0) & \text { for } k \in \mathbf{Z}_{+}, \\ \left(2^{\prime \prime}\right) & (2 k+1,2, \ldots, 2, & 1, & 0, & 0, \ldots, 0) & \text { for } k \in \mathbf{Z}_{+}, \\ \left(3^{\prime \prime}\right) & (2 k, 2, \ldots, 2, & 1, & 1, & 0, \ldots, 0) & \text { for } k \in \mathbf{Z}_{+}, \\ \left(4^{\prime \prime}\right) & (2 k, 2, \ldots, 2, & 2, & 0, & 0, \ldots, 0) & \text { for } k \in \mathbf{Z}_{+}, \\ \left(5^{\prime \prime}\right) & (2 k+1,2, \ldots, 2, & 2, & 1, & 0, \ldots, 0) & \text { for } k \in \mathbf{Z}_{+}, \\ \left(6^{\prime \prime}\right) & (2 k, 2, \ldots, 2, & 2, & 2, & 0, \ldots, 0) & \text { for } k \in \mathbf{Z}_{+} .\end{array}$

The only $n$-tuples in $Z^{n}$ of the form $\left(1^{\prime \prime}\right)-\left(6^{\prime \prime}\right)$ satisfying (v) are $i$ th $\quad(i+1)$ th

$\begin{array}{lllll}(0, \ldots, & 0, & 0, & \ldots, 0) & \text { with } \tilde{m}_{\lambda}(\mu)=1 \text {, } \\ (1, \ldots, & 1, & 0, & \ldots, 0) & \text { with } \tilde{m}_{\lambda}(\mu)=1 \text {, } \\ (0, \ldots, & 1, & 1, & \ldots, 0) & \text { with } \tilde{m}_{\lambda}(\mu)=1 \text {, } \\ (2, \ldots, & 2, & 0, & \ldots, 0) & \text { with } \tilde{m}_{\lambda}(\mu)=1 \text {, } \\ (1, \ldots, & 2, & 1, & \ldots, 0) & \text { with } \tilde{m}_{\lambda}(\mu)=1 \text {, } \\ (0, \ldots, & 2, & 2, & \ldots, 0) & \text { with } \tilde{m}_{\lambda}(\mu)=1 .\end{array}$

We omit the computation which is straightforward as in the previous case.

Set $b_{0}^{j}=\left(a_{n+1}+b_{1}-2_{j}\right) / 2$ for $j=0, \ldots, \min \left(b_{1}, a_{n+1}\right)$ and $b_{1}=0,1,2$. If $b_{1}=0$, then $j=0$ and $b_{0}^{0}=(n-i)$. If $b_{1}=1$, then $j=0,1$ and $b_{0}^{0}=(n-i)+\frac{1}{2}$, $b_{0}^{1}=(n-i)-\frac{1}{2}$. If $b_{1}=2$, then $j=0,1,2$ and $b_{0}^{0}=n-i+1, b_{0}^{1}=n-i, b_{0}^{2}=$ $n-i-1$. Therefore the $M$-types are the ones listed.

(4) If $i=n$, then $a_{1}=\cdots=a_{n}=2$ and $a_{n+1}=0$. The $n$-tuples in $\mathbf{Z}^{n}$ satisfying (i)-(iv) are

$\begin{array}{lll}\left(1^{\prime \prime \prime}\right) & (2 k, 2, \ldots, 2,0) & \text { for } k \in \mathbf{Z}_{+}, \\ \left(2^{\prime \prime \prime}\right) & (2 k+1,2, \ldots, 2,1) & \text { for } k \in \mathbf{Z}_{+}, \\ \left(3^{\prime \prime \prime}\right) & (2 k, 2, \ldots, 2,2) & \text { for } k \in \mathbf{Z}_{+},\end{array}$

and the ones satisfying (i)-(v) are $(0,2, \ldots, 2,0),(1,2, \ldots, 2,1)$ and $(2,2, \ldots, 2)$ all with multiplicity one.

Since $\min \left(a_{n+1}, b_{1}\right)=0$ for $b_{1}=0,1,2$, then we have $j=0$ always and

$$
\begin{aligned}
& \text { if } b_{1}=0, \quad \text { then } b_{0}^{0}=0 ; \\
& \text { if } b_{1}=1, \quad \text { then } b_{0}^{0}=\frac{1}{2} ; \\
& \text { if } b_{1}=2,
\end{aligned}
$$

The theorem is now completely proved.

THEOREM 12.5. Let $G=F_{4}$. Let $\phi$ be as in 10.8 and if $\mu=\sum_{i=1}^{3} a_{i} \varepsilon_{i}$, write $\mu=\left(a_{1}, a_{2}, a_{3}\right)$.

(1) The $M$-types of $\tau_{2 \delta_{n}^{F_{1}}}$ are $\pi_{\mu_{j}^{\prime}} \circ \phi$ for $j=1, \ldots, 10$ and

$$
\begin{array}{llll}
\mu_{1}^{1}=(3,1,1), & \mu_{2}^{1}=(2,1,1), & \mu_{3}^{1}=(1,1,1), & \mu_{4}^{1}=\left(\frac{5}{2}, \frac{3}{2}, \frac{1}{2}\right), \\
\mu_{5}^{1}=\left(\frac{5}{2}, \frac{1}{2}, \frac{1}{2}\right), & \mu_{6}^{1}=\left(\frac{3}{2}, \frac{3}{2}, \frac{1}{2}\right), & \mu_{7}^{1}=\left(\frac{3}{2}, \frac{1}{2}, \frac{1}{2}\right), & \mu_{8}^{1}=(2,2,0), \\
\mu_{9}^{1}=(2,1,0), & \mu_{10}^{1}=(2,0,0) . &
\end{array}
$$


They all have multiplicity one.

(2) The M-types of $\tau_{2 \delta_{n}^{F_{2}}}$ are $\pi_{\mu_{j}^{2}} \circ \phi$ for $j=1, \ldots, 18$, where, unless otherwise stated, the multiplicity is one, and

$$
\begin{aligned}
& \mu_{1}^{2}=(2,2,0), \quad \mu_{2}^{2}=(2,2,1), \quad \mu_{3}^{2}=(2,2,2), \quad \mu_{4}^{2}=\left(\frac{3}{2}, \frac{3}{2}, \frac{3}{2}\right)\left(m_{\lambda}\left(\mu_{4}^{2}\right)=2\right), \\
& \mu_{5}^{2}=\left(\frac{3}{2}, \frac{3}{2}, \frac{1}{2}\right)\left(m_{\mu}\left(\mu_{5}^{2}\right)=2\right), \quad \mu_{6}^{2}=(1,1,1)\left(m_{\lambda}\left(\mu_{6}^{2}\right)=3\right), \\
& \mu_{7}^{2}=\left(\frac{5}{2}, \frac{3}{2}, \frac{1}{2}\right), \quad \mu_{8}^{2}=\left(\frac{5}{2}, \frac{3}{2}, \frac{3}{2}\right), \quad \mu_{9}^{2}=(2,1,0), \quad \mu_{10}^{2}=(2,1,1)\left(m_{\lambda}\left(\mu_{10}^{2}\right)=2\right), \\
& \mu_{11}^{2}=(1,1,0), \quad \mu_{12}^{2}=\left(\frac{3}{2}, \frac{1}{2}, \frac{1}{2}\right)\left(m_{\lambda}\left(\mu_{12}^{2}\right)=2\right), \\
& \mu_{13}^{2}=\left(\frac{1}{2}, \frac{1}{2}, \frac{1}{2}\right)\left(m_{\lambda}\left(\mu_{13}^{2}\right)=2\right), \quad \mu_{14}^{2}=(3,1,1), \quad \mu_{15}^{2}=\left(\frac{5}{2}, \frac{1}{2}, \frac{1}{2}\right), \\
& \mu_{16}^{2}=(2,0,0), \quad \mu_{17}^{2}=(1,0,0), \quad \mu_{18}^{2}=(0,0,0) .
\end{aligned}
$$

(3) The M-types of $\tau_{2 \delta^{F_{3}}}$ are $\pi_{\mu_{j}^{3}} \circ \phi$ for $j=1, \ldots, 5$ and $\mu_{1}^{3}=(2,2,2), \quad \mu_{2}^{3}=\left(\frac{3}{2}, \frac{3}{2}, \frac{3}{2}\right), \quad \mu_{3}^{3}=(1,1,1), \mu_{4}^{3}=\left(\frac{1}{2}, \frac{1}{2}, \frac{1}{2}\right), \mu_{5}^{3}=(0,0,0)$.

They all have multiplicity one.

Proof. We first prove (1).

$$
2 \delta_{n}^{F_{1}}=2 \varepsilon+2 \varepsilon_{2}+2 \varepsilon_{3}=\sum_{i=1}^{4} a_{i} \varepsilon_{i} \quad \text { with } a_{1}=a_{2}=a_{3}=2, a_{4}=0
$$

Let $\left(q_{1}, \ldots, q_{4}\right)$ be a 4-tuple satisfying:

(i) $q_{i}-q_{j} \in \mathbf{Z}, 2 q_{i} \in \mathbf{Z}$,

(ii) $q_{1} \geqslant q_{2} \geqslant q_{3} \geqslant\left|q_{4}\right|$,

(iii) $a_{1} \geqslant q_{1} \geqslant \cdots \geqslant q_{3} \geqslant a_{4} \geqslant\left|q_{4}\right|$,

(iv) $a_{i}-q_{j} \in \mathbf{Z}$.

Since $a_{i} \in \mathbf{Z}$, then condition (iv) is equivalent to $q_{i} \in \mathbf{Z}$. If $q_{i} \in \mathbf{Z}$, then (iii) forces $q_{1}=q_{2}=2, q_{3}=2,1,0$ and $q_{4}=0$. So the 4-tuples $\left(q_{1}, \ldots, q_{4}\right)$ satisfying (i)-(iv) are $(2,2,2,0),(2,2,1,0)$ and $(2,2,0,0)$.

Set $\left(q_{1}^{\prime}, \ldots, q_{4}^{\prime}\right)=\phi\left(q_{1}, \ldots, q_{4}\right)$. Let $b_{1}, b_{2}, b_{3}$ be a 3-tuple satisfying:

(i) $b_{i}-b_{j} \in \mathbf{Z}, 2 b_{i} \in \mathbf{Z}$,

(ii) $b_{1} \geqslant b_{2} \geqslant b_{3} \geqslant 0$,

(iii) $q_{1}^{\prime} \geqslant b_{1} \geqslant q_{2}^{\prime} \geqslant b_{2} \geqslant q_{3}^{\prime} \geqslant b_{3} \geqslant\left|q_{4}^{\prime}\right|$,

(iv) $q_{i}^{\prime}-b_{j} \in \mathbf{Z}$.

(1') If $\left(q_{1}, \ldots, q_{4}\right)=(2,2,2,0)$, then $\left(q_{1}^{\prime}, \ldots, q_{4}^{\prime}\right)=(3,1,1,1)$ and $\left(b_{1}, b_{2}, b_{3}\right)$ is equal to $(3,1,1),(2,1,1)$ or $(1,1,1)$.

$\left(2^{\prime}\right)$ If $\left(q_{1}, \ldots, q_{4}\right)=(2,2,1,0)$, then $\left(q_{1}^{\prime}, \ldots, q_{4}^{\prime}\right)=\left(\frac{5}{2}, \frac{3}{2}, \frac{1}{2}, \frac{1}{2}\right)$ and $\left(b_{1}, b_{2}, b_{3}\right)$ is equal to $\left(\frac{5}{2}, \frac{3}{2}, \frac{1}{2}\right),\left(\frac{5}{2}, \frac{1}{2}, \frac{1}{2}\right),\left(\frac{3}{2}, \frac{3}{2}, \frac{1}{2}\right)$ or $\left(\frac{3}{2}, \frac{1}{2}, \frac{1}{2}\right)$.

(3') If $\left(q_{1}, \ldots, q_{4}\right)=(2,2,0,0)$, then $\left(q_{1}^{\prime}, \ldots, q_{4}^{\prime}\right)=(2,2,0,0)$ and $\left(b_{1}, b_{2}, b_{3}\right)$ is equal to $(2,2,0),(2,1,0)$ or $(2,0,0)$.

Hence by Theorem 10.9 , the $M$-types are the ones described.

We now prove (2).

$$
2 \delta_{n}^{F_{2}}=\sum_{i=1}^{4} a_{i} \varepsilon_{i} \quad \text { with } a_{1}=3, a_{2}=a_{3}=a_{4}=1 .
$$


Let $\left(q_{1}, \ldots, q_{4}\right)$ be as in the proof of (1) satisfying (i)-(iv). Since $a_{i} \in \mathbf{Z}$ forces $q_{i} \in \mathbf{Z}$, then $\left(q_{1}, \ldots, q_{4}\right)$ is either one of the following:

$$
\begin{aligned}
& (3,1,1,1), \quad(2,1,1,1), \quad(1,1,1,1), \\
& (3,1,1,0), \quad(2,1,1,0), \quad(1,1,1,0), \\
& (3,1,1,-1), \quad(2,1,1,-1), \quad(1,1,1,-1) .
\end{aligned}
$$

Let $\left(b_{1}, b_{2}, b_{3}\right)$ satisfying (i)-(iv) be as in the proof of (1).

(1') If $\left(q_{1}, \ldots, q_{4}\right)=(3,1,1,1)$, then $\left(q_{1}^{\prime}, \ldots, q_{4}^{\prime}\right)=(2,2,2,0)$ and $\left(b_{1}, b_{2}, b_{3}\right)$ is equal to $(2,2,2),(2,2,1)$ or $(2,2,0)$.

(2') If $\left(q_{1}, \ldots, q_{4}\right)=(2,1,1,1)$, then $\left(q_{1}^{\prime}, \ldots, q_{4}^{\prime}\right)=\left(\frac{3}{2}, \frac{3}{2}, \frac{3}{2}, \frac{1}{2}\right)$ and $\left(b_{1}, b_{2}, b_{3}\right)$ is equal to $\left(\frac{3}{2}, \frac{3}{2}, \frac{3}{2}\right)$ or $\left(\frac{3}{2}, \frac{3}{2}, \frac{1}{2}\right)$.

(3') If $\left(q_{1}, \ldots, q_{4}\right)=(1,1,1,1)$, then $\left(q_{1}^{\prime}, \ldots, q_{4}^{\prime}\right)=(1,1,1,1)$ and $\left(b_{1}, b_{2}, b_{3}\right)$ $=(1,1,1)$.

(4) If $\left(q_{1}, \ldots, q_{4}\right)=(3,1,1,0)$, then $\left(q_{1}^{\prime}, \ldots, q_{4}^{\prime}\right)=\left(\frac{5}{2}, \frac{3}{2}, \frac{3}{2},-\frac{1}{2}\right)$ and $\left(b_{1}, b_{2}, b_{3}\right)$ is equal to $\left(\frac{5}{2}, \frac{3}{2}, \frac{3}{2}\right),\left(\frac{5}{2}, \frac{3}{2}, \frac{1}{2}\right),\left(\frac{3}{2}, \frac{3}{2}, \frac{3}{2}\right)$ or $\left(\frac{3}{2}, \frac{3}{2}, \frac{1}{2}\right)$.

$\left(5^{\prime}\right)$ If $\left(q_{1}, \ldots, q_{4}\right)=(2,1,1,0)$, then $\left(q_{1}^{\prime}, \ldots, q_{4}^{\prime}\right)=(2,1,1,0)$ and $\left(b_{1}, b_{2}, b_{3}\right)$ is equal to $(2,1,1),(2,1,0),(1,1,1)$ or $(1,1,0)$.

(6') If $\left(q_{1}, \ldots, q_{4}\right)=(1,1,1,0)$, then $\left(q_{1}^{\prime}, \ldots, q_{4}^{\prime}\right)=\left(\frac{3}{2}, \frac{1}{2}, \frac{1}{2}, \frac{1}{2}\right)$ and $\left(b_{1}, b_{2}, b_{3}\right)$ is equal to $\left(\frac{3}{2}, \frac{1}{2}, \frac{1}{2}\right)$ or $\left(\frac{1}{2}, \frac{1}{2}, \frac{1}{2}\right)$.

( $\left.7^{\prime}\right)$ If $\left(q_{1}, \ldots, q_{4}\right)=(3,1,1,-1)$, then $\left(q_{1}^{\prime}, \ldots, q_{4}^{\prime}\right)=(3,1,1,-1)$ and $\left(b_{1}, b_{2}, b_{3}\right)$ is equal to $(3,1,1),(2,1,1)$ or $(1,1,1)$.

$\left(8^{\prime}\right)$ If $\left(q_{1}, \ldots, q_{4}\right)=(2,1,1,-1)$, then $\left(q_{1}^{\prime}, \ldots, q_{4}^{\prime}\right)=\left(\frac{5}{2}, \frac{1}{2}, \frac{1}{2},-\frac{1}{2}\right)$ and $\left(b_{1}, b_{2}, b_{3}\right)$ is equal to $\left(\frac{5}{2}, \frac{1}{2}, \frac{1}{2}\right),\left(\frac{3}{2}, \frac{1}{2}, \frac{1}{2}\right)$ or $\left(\frac{1}{2}, \frac{1}{2}, \frac{1}{2}\right)$.

$\left(9^{\prime}\right)$ If $\left(q_{1}, \ldots, q_{4}\right)=(1,1,1,-1)$, then $\left(q_{1}^{\prime}, \ldots, q_{4}^{\prime}\right)=(2,0,0,0)$ and $\left(b_{1}, b_{2}, b_{3}\right)$ is equal to $(2,0,0),(1,0,0)$ or $(0,0,0)$.

Finally, we prove (3).

$$
2 \delta_{n}^{F_{3}}=\sum_{i=1}^{4} a_{i} \varepsilon_{i} \quad a_{1}=4, a_{2}=a_{3}=a_{4}=0 .
$$

Let $\left(q_{1}, \ldots, q_{4}\right)$ be a 4-tuple as in the proof of (1) satisfying (i)-(iv). Since $a_{i} \in \mathbf{Z}$, then $\left(q_{1}, \ldots, q_{4}\right)$ is either one of the following $(4,0,0,0),(3,0,0,0),(2,0,0,0)$, $(1,0,0,0)$ or $(0,0,0,0)$.

Let $\left(b_{1}, b_{2}, b_{3}\right)$ satisfy (i)-(iv) as usual.

$\left(1^{\prime}\right)$ If $\left(q_{1}, \ldots, q_{4}\right)=(4,0,0,0)$, then $\left(q_{1}^{\prime}, \ldots, q_{4}^{\prime}\right)=(2,2,2,-2)$ and $\left(b_{1}, b_{2}, b_{3}\right)=(2,2,2)$.

$\left(2^{\prime}\right)$ If $\left(q_{1}, \ldots, q_{4}\right)=(3,0,0,0)$, then $\left(q_{1}^{\prime}, \ldots, q_{4}^{\prime}\right)=\left(\frac{3}{2}, \frac{3}{2}, \frac{3}{2},-\frac{3}{2}\right)$ and $\left(b_{1}, b_{2}, b_{3}\right)=\left(\frac{3}{2}, \frac{3}{2}, \frac{3}{2}\right)$.

(3') If $\left(q_{1}, \ldots, q_{4}\right)=(2,0,0,0)$, then $\left(q_{1}^{\prime}, \ldots, q_{4}^{\prime}\right)=(1,1,1,-1)$ and $\left(b_{1}, b_{2}, b_{3}\right)=(1,1,1)$.

(4) If $\left(q_{1}, \ldots, q_{4}\right)=(1,0,0,0)$, then $\left(q_{1}^{\prime}, \ldots, q_{4}^{\prime}\right)=\left(\frac{1}{2}, \frac{1}{2}, \frac{1}{2},-\frac{1}{2}\right)$ and $\left(b_{1}, b_{2}, b_{3}\right)=\left(\frac{1}{2}, \frac{1}{2}, \frac{1}{2}\right)$.

$\left(5^{\prime}\right)$ If $\left(q_{1}, \ldots, q_{4}\right)=(0,0,0,0)$, then $\left(q_{1}^{\prime}, \ldots, q_{4}^{\prime}\right)=(0,0,0,0)$ and $\left(b_{1}, b_{2}, b_{3}\right)$ $=(0,0,0)$. 


\section{Some result on positive root systems.}

13.1. (Notation as in $\$ 10$.) In order to use the equation of the infinitesimal character, as we pointed out in 11.1, we need to classify all the positive root systems for $\Phi$, containing $\alpha$ and $\Phi_{m}^{+}$. We do it by a case by case argument.

13.2. $G=\operatorname{Spin}(2 n, 1)$. Set $P^{0,1}=\Phi^{+}$and $\gamma_{i}^{1}=\gamma_{i}, 1<i<n$. Define $P^{0,2}=$ $s_{\gamma_{1}^{1}} P^{0,1}$ and $\gamma_{i}^{2}=s_{\gamma_{1}^{1}} \gamma_{i}^{1}$, for $i=1, \ldots, n$.

Suppose $P^{0, j}$ has been defined with simple roots $\gamma_{1}^{j}, \ldots, \gamma_{n}^{j}$. Then $P^{0, j+1}=$ $s_{\gamma_{j}^{j}} P^{0, j}$ and $\gamma_{i}^{j+1}=s_{\gamma_{j}^{j}} \gamma_{i}^{j}$.

LEMMA 13.3. (1) $P^{0, j}, 1 \leqslant j \leqslant n$, contains $\Phi_{m}^{+}$.

(2) $P^{0, j}$ contains $\alpha=\gamma_{1}+\cdots+\gamma_{n}$ the real root in $P^{0,1}$, if $j \leqslant n$.

Proof. $\left(1^{\prime}\right) \gamma_{j}^{j}=\gamma_{1}+\cdots+\gamma_{j}$, if $j \leqslant n$.

Suppose that we have shown that $\gamma_{j}^{j}=\gamma_{1}+\cdots+\gamma_{j}$. (This is clearly true for $j=1$.) Then $\gamma_{j+1}^{j+1}=s_{\gamma_{j}^{\prime}} \gamma_{j+1}^{j}=s_{\gamma_{j}^{j} s_{\gamma_{-1}^{j}-1}} \cdots s_{\gamma_{1}} \gamma_{j+1}^{1}=s_{\gamma \gamma_{j+1}^{\prime}}=\gamma_{1}+\cdots+\gamma_{j+1}$. This completes the induction and proves $\left(1^{\prime}\right)$.

(2') $\gamma_{j}^{j} \notin \Phi_{m}$, if $j \leqslant n$.

Clearly $\gamma_{1}^{1}=\gamma_{1} \notin \Phi_{m}$. Suppose that $\gamma_{j}^{j} \notin \Phi_{m}$, then by $\left(1^{\prime}\right), \gamma_{j+1}^{j+1}=\gamma_{j}^{j}+\gamma_{j+1}$. Since $\gamma_{j}^{j} \notin \Phi_{m}$ and $\gamma_{j+1} \in \Phi_{m}$ we see that $\gamma_{j+1}^{j+1} \notin \Phi_{m}$.

Now $P^{0, j+1}=\left(P^{0, j}-\left\{\gamma_{j}^{j}\right\}\right) \cup\left\{-\gamma_{j}^{j}\right\}$. Hence if $\Phi_{m}^{+} \subset P^{0, j}$, then $\Phi_{m}^{+} \subset P^{0, j+1}$, for $j \leqslant n$, by $\left(2^{\prime}\right)$. Since $\Phi_{m}^{+} \subset P^{0,1}$ we have thus shown that $\Phi_{m}^{+} \subset P^{0, j}$, for $j<n$, and (1) is true.

By $\left(1^{\prime}\right) \alpha=\gamma_{j}^{j}+\gamma_{j+1}+\cdots+\gamma_{n}$ if $j \leqslant n-2, \alpha=\gamma_{n-1}^{n-1}+\gamma_{n}$ if $j=n-1$, and $\alpha=\gamma_{n}^{n}$ if $j=n$.

Since $\gamma_{i}=\gamma_{i}^{j}$, if $i>j$, we see that $\alpha \in P^{0, j}$, for $j=1, \ldots, n$.

LEMMA 13.4. The systems of positive roots containing $\Phi_{m}^{+} \cup\{\alpha\}$ are precisely $P^{0,1}, \ldots, P^{0, n}$.

Proof. It is easy to see that $P^{0,1}, \ldots, P^{0, n}$ are all distinct. Since there are exactly $|W(\Phi)| / 2\left|W\left(\Phi_{m}\right)\right|$ systems of positive roots containing $\Phi_{m}^{+} \cup\{\alpha\}$, the lemma follows.

13.5. $G=\operatorname{SU}(n, 1)$. We use the notation $\Phi^{+}: \tilde{\varepsilon}_{1} \geqslant \cdots \geqslant \tilde{\varepsilon}_{n+1}$ for the fundamental Weyl chamber relative to $\Phi^{+}$.

We define $P^{i, j}$ a system of positive roots for $\Phi, 0 \leqslant i \leqslant n-1,1 \leqslant j \leqslant n-i$, as follows:

$$
P^{0,1}=\Phi^{+}, \quad \gamma_{k}^{0,1}=\gamma_{k}, \quad k=1, \ldots, n .
$$

Suppose that $P^{i, j}, \gamma_{1}^{i j}, \ldots, \gamma_{n}^{i, j}$ have been defined; then

$$
\begin{gathered}
P^{i+1, j}=s_{\gamma_{i+1}^{i j}} P^{i, j} \text { and } \gamma_{k}^{i+1, j}=s_{\gamma_{i+1}^{i j}} \gamma_{k}^{i, j}, \\
P^{i, j+1}=s_{\gamma_{n}^{i j} j+1} P^{i, j} \text { and } \gamma_{k}^{i, j+1}=s_{\gamma_{n}^{i j} j+1} \gamma_{k}^{i, j} .
\end{gathered}
$$

LEMMA 13.6. (1) The systems of positive roots $P^{i j}$ are well defined and distinct.

(2) $P^{i j}$ contains $\Phi_{m}^{+}$and $\alpha$, where $\alpha=\tilde{\varepsilon}_{1}-\tilde{\varepsilon}_{n+1}$ is the real root of $\phi^{+}$.

(3) If $Q \subseteq \Phi$ is a system of positive roots such that $\{\alpha\} \cup\left\{\Phi_{m}^{+}\right\} \subset Q$, then $Q=P^{i, j}$ for some $i, j$. 
Proof. (1') If $2 \leqslant i+j<n+1$, then $\gamma_{i}^{i-1, j}=\gamma_{1}+\cdots+\gamma_{i}=\tilde{\varepsilon}_{1}-\tilde{\varepsilon}_{i+1}$. Indeed $\gamma_{1}^{0,1}=\gamma_{1}$. Suppose that $\left(1^{\prime}\right)$ is true for $2 \leqslant i+j \leqslant k$; then we prove it for $i+j=k+1$.

$$
\gamma_{i}^{i-1, j}=s_{\gamma_{i-1}^{i}-2 j} \gamma_{i}^{i-2 j}=s_{\gamma_{i=1}^{i-2 j}} \ldots s_{\gamma_{1}^{0}} \gamma_{i}^{0, j} .
$$

But $\gamma_{i}^{0, j}=\gamma_{i}$, so $\gamma_{i}^{i-1, j}=\gamma_{1}+\cdots+\gamma_{i}$. This completes the induction and proves (1'). Similarly

$$
\gamma_{n-j+1}^{i, j}=\tilde{\varepsilon}_{n-j+1}-\tilde{\varepsilon}_{n+1}=\gamma_{n-j+1}+\cdots+\gamma_{n}, \quad \text { if } 2<i+j<n+1 .
$$

Also

$$
\begin{aligned}
P^{i, j}: \tilde{\varepsilon}_{2} & \geqslant \tilde{\varepsilon}_{3} \geqslant \cdots \geqslant \tilde{\varepsilon}_{i+1} \geqslant \tilde{\varepsilon}_{1} \geqslant \tilde{\varepsilon}_{i+2} \geqslant \cdots \geqslant \tilde{\varepsilon}_{n-j+1} \\
& \geqslant \tilde{\varepsilon}_{n+1} \geqslant \tilde{\varepsilon}_{n-j+2} \geqslant \cdots \geqslant \tilde{\varepsilon}_{n}
\end{aligned}
$$

if $1 \leqslant i \leqslant n, 2 \leqslant j \leqslant n-i$.

$$
\begin{aligned}
& P^{0, i}: \tilde{\varepsilon}_{1} \geqslant \cdots \geqslant \tilde{\varepsilon}_{n-i+1} \geqslant \tilde{\varepsilon}_{n+1} \geqslant \tilde{\varepsilon}_{n-i+2} \geqslant \cdots \geqslant \tilde{\varepsilon}_{n}, \quad i \geqslant 2, \\
& P^{i, 1}: \tilde{\varepsilon}_{2} \geqslant \cdots \geqslant \tilde{\varepsilon}_{i+1} \geqslant \tilde{\varepsilon}_{1} \geqslant \tilde{\varepsilon}_{i+2} \geqslant \cdots \geqslant \tilde{\varepsilon}_{n+1}, \quad i \geqslant 1 .
\end{aligned}
$$

Another way of stating $\left(3^{\prime}\right)$ is that $P^{i, j}$ has $\tilde{\varepsilon}_{1}$ in the $(i+1)$ th position and $\tilde{\varepsilon}_{n+1}$ in the $(n+2-j)$ th position and $\tilde{\varepsilon}_{2} \geqslant \cdots \geqslant \tilde{\varepsilon}_{n}$. Suppose that we have proved this for $1 \leqslant i+j \leqslant k$. We prove it then for $i+j=k+1$. If $i+j=k<n$, by (1') it follows that

$$
\begin{aligned}
P^{i+1, j} & =s_{\gamma_{i+1}^{i j}} P^{i, j}: \tilde{\varepsilon}_{2} \geqslant \cdots \geqslant \tilde{\varepsilon}_{i+1} \geqslant \tilde{\varepsilon}_{i+2} \geqslant \tilde{\varepsilon}_{1} \geqslant \tilde{\varepsilon}_{i+3} \geqslant \cdots \geqslant \tilde{\varepsilon}_{n-j+1} \\
& \geqslant \tilde{\varepsilon}_{n+1} \geqslant \cdots \geqslant \tilde{\varepsilon}_{n-j+2} \geqslant \cdots \geqslant \tilde{\varepsilon}_{n}
\end{aligned}
$$

which is correct.

Similarly by $\left(2^{\prime}\right), P^{i, j+1}=s_{\gamma_{n}^{i j} j+1} P^{i, j}$ has $\tilde{\varepsilon}_{1}$ in the $(i+1)$ th position, $\tilde{\varepsilon}_{n+1}$ in the $(n-j+1)$ th position and $\tilde{\varepsilon}_{2} \geqslant \cdots \geqslant \tilde{\varepsilon}_{n}$, since $s_{\gamma_{n}^{j} j+1}$ interchanges $\tilde{\varepsilon}_{n+1}$ with $\tilde{\varepsilon}_{n-j+1}$.

This proves (3') and the lemma follows immediately since $\alpha=\tilde{\varepsilon}_{1}-\tilde{\varepsilon}_{n+1}$ and the $P^{i, j}$ are $n(n+1) / 2$ and hence exhaust all systems of positive roots containing $\Phi_{m}^{+}$ and $\alpha$.

13.7. $G=\operatorname{Sp}(n, 1)$. We label $\Phi^{+}$by $\tilde{\varepsilon}_{1} \geqslant \tilde{\varepsilon}_{2} \geqslant \cdots \geqslant \tilde{\varepsilon}_{n+1} \geqslant 0$, as usual. The real root in $\Phi^{+}$is $\alpha=\tilde{\varepsilon}_{1}+\tilde{\varepsilon}_{2}$. Thus if $Q$ is a system of positive roots and $Q \supset \Phi_{m}^{+} \cup\{\alpha\}, Q$ must have

$$
\tilde{\varepsilon}_{1} \geqslant \pm \tilde{\varepsilon}_{2}, \quad \tilde{\varepsilon}_{3} \geqslant \cdots \geqslant \tilde{\varepsilon}_{n+1}>0 .
$$

All systems of positive roots are given by

$$
n_{1} \tilde{\varepsilon}_{\sigma(1)} \geqslant n_{2} \tilde{\varepsilon}_{\sigma(2)} \geqslant \cdots \geqslant n_{n+1} \tilde{\varepsilon}_{\sigma(n+1)} \geqslant 0 \text { with } n_{i}= \pm 1, \sigma \in S_{n+1} .
$$

Let $P^{i, j}, 0 \leqslant i<j \leqslant n$ be the unique system of the form (I) with $\tilde{\varepsilon}_{1}$ in the $(i+1)$ th position and $\tilde{\varepsilon}_{2}$ in the $(j+1)$ th position and $\tilde{\varepsilon}_{3}>\cdots>\tilde{\varepsilon}_{n+1}>0$, i.e. $\tilde{\varepsilon}_{3} \geqslant \cdots \geqslant \tilde{\varepsilon}_{i+2} \geqslant \tilde{\varepsilon}_{1} \geqslant \tilde{\varepsilon}_{i+3} \geqslant \cdots \geqslant \tilde{\varepsilon}_{j} \geqslant \tilde{\varepsilon}_{j+1} \geqslant \tilde{\varepsilon}_{2} \geqslant \tilde{\varepsilon}_{j+2} \geqslant \cdots \geqslant \tilde{\varepsilon}_{n+1}>0$.

For $0 \leqslant i \leqslant n-1,1 \leqslant j \leqslant n-i$, let $P^{i j+n}$ be the unique system of positive roots of the form (I) with $\tilde{\varepsilon}_{1}$ in the $(i+1)$ th position and $-\tilde{\varepsilon}_{2}$ in the $(n+2-j)$ th 
position and $\tilde{\varepsilon}_{3} \geqslant \cdots \geqslant \tilde{\varepsilon}_{n+1} \geqslant 0$. Clearly there are precisely $2 \sum_{i=0}^{n-1}(n-i)=2 n^{2}$ $-2(n-1) n / 2=n^{2}+n=n(n+1)$ such systems $P^{i, j}$ and they are all distinct.

LEMMA 13.8. (1) The $P^{i, j}$ exhaust all the systems of positive roots containing $\Phi_{m}^{+}$ and $\{\alpha\}$.

(2) If $i, j, i^{\prime}, j^{\prime}$ are in the range of definition of $P^{i, j}$, then if there is $\gamma \in P^{i, j}, \gamma$ simple in $P^{i, j}$, such that $s_{\gamma} P^{i, j}=P^{i^{\prime} j^{\prime}}$, then $\left|i-i^{\prime}\right|+\left|j-j^{\prime}\right|=1$.

Proof. The $P^{i, j}$ are clearly distinct.

$$
\left[W(\Delta): W\left(\Delta_{m}\right) \cdot\left\{1, s_{\alpha}\right\}\right]=\frac{2^{n+1}(n+1) !}{2^{n+1}(n-1) !}=(n+1) n
$$

Hence (1). (2) is proved by inspection.

Note. Schematically condition (2) says that if we label the systems of positive roots $P^{i j}$ by their Cartesian coordinates and if we draw a line between two such systems related by simple roots, then we have:

Diagram for $\operatorname{Sp}(n, 1)$ :
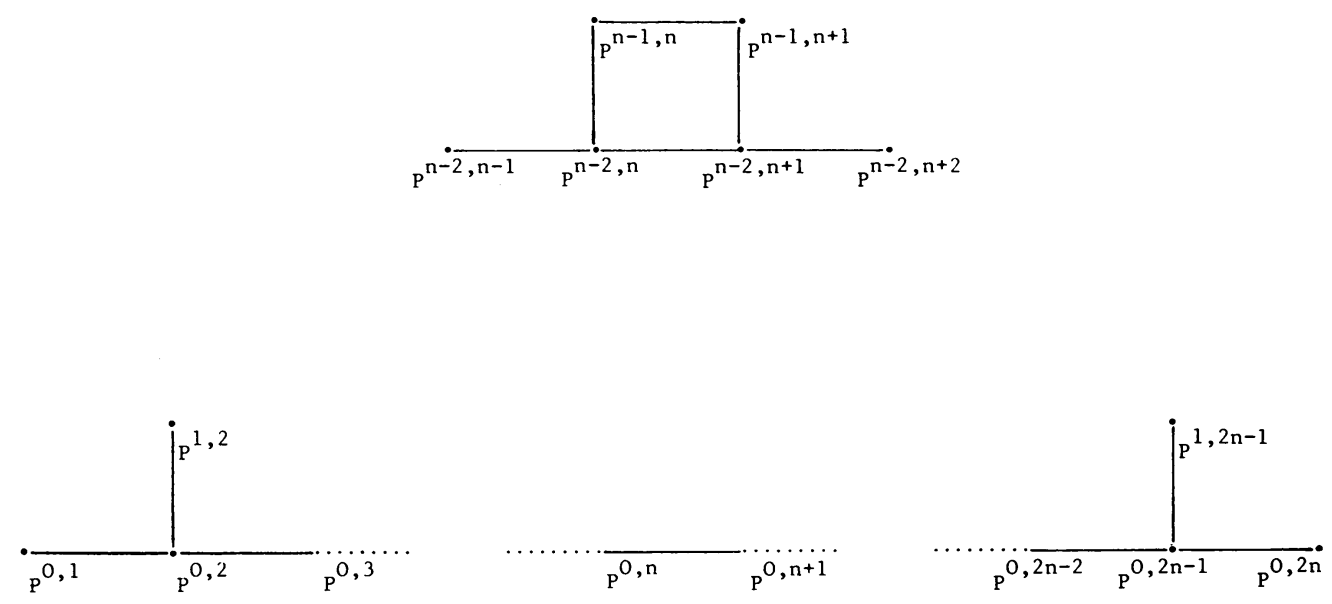

REMARK. (2) of Lemma 13.8 is valid also for $\operatorname{Spin}(2 n, 1)$ and $\operatorname{SU}(n, 1)$. We thus have

Diagram for $\operatorname{Spin}(2 n, 1)$ :

$\overline{\mathrm{P}^{0,1}} \cdot \overline{\mathrm{P}^{0,2}} \cdot \cdots \cdots \cdots \cdots \cdots \cdot \cdot \overline{\mathrm{P}^{0, \mathrm{n}-1}} \cdot \mathrm{P}^{\mathrm{0,n}}$


Diagram for $\operatorname{SU}(n, 1)$ :

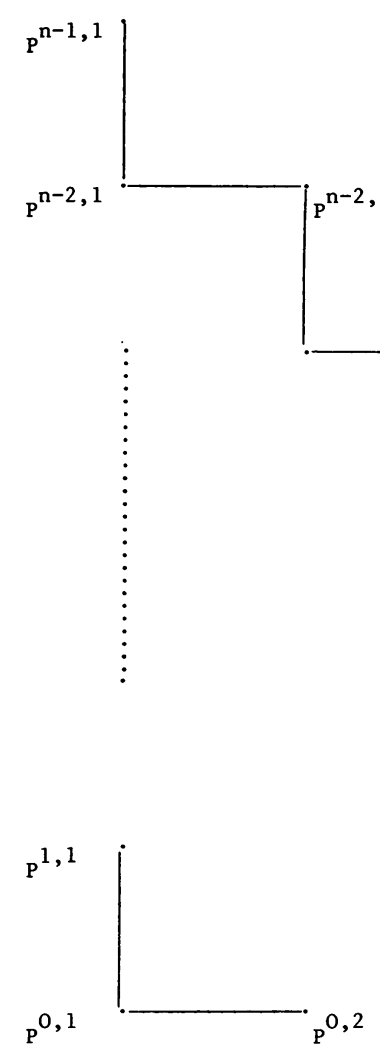

$P^{n-3,3}$

13.9. $G=F_{4}$. For want of a better way we give a list of the $12=$ $|W(\Phi)| / 2\left|W\left(\Phi_{m}\right)\right|$ systems of positive roots that contain $\Phi_{m}^{+}$and $\alpha$. Set $P^{0,1}=\Phi^{+}$, then:

$$
\begin{aligned}
& \mathrm{P}^{0,2}=\mathrm{s}_{\gamma_{4}} \mathrm{P}^{0,1}
\end{aligned}
$$

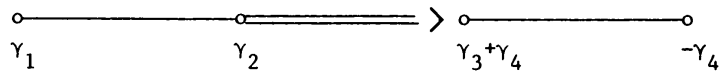

$$
\begin{aligned}
& \mathrm{P}^{0,3}=\mathrm{s}_{\gamma_{3}+\gamma_{4}} \mathrm{P}^{0,2}
\end{aligned}
$$

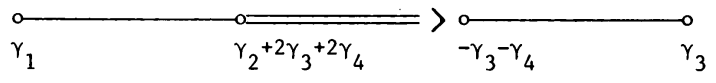

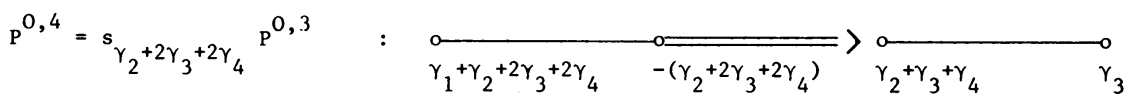

$$
\begin{aligned}
& \mathrm{P}^{0,5}=\mathrm{s}_{\gamma_{1}+\gamma_{2}+2 \gamma_{3}+2 \gamma_{4}} \mathrm{P}^{0,4}: \quad \overbrace{-\left(\gamma_{1}+\gamma_{2}+2 \gamma_{3}+2 \gamma_{4}\right) \gamma_{1}}^{0}>\underset{\gamma_{2}+\gamma_{3}+\gamma_{4}}{\circ} \overbrace{\gamma_{3}}
\end{aligned}
$$




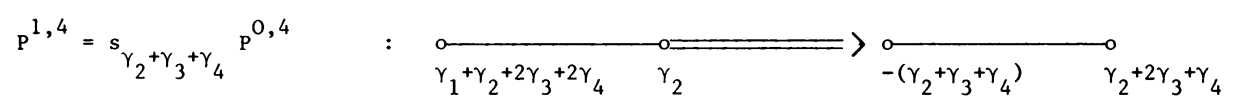

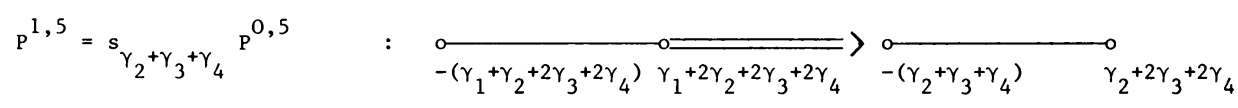

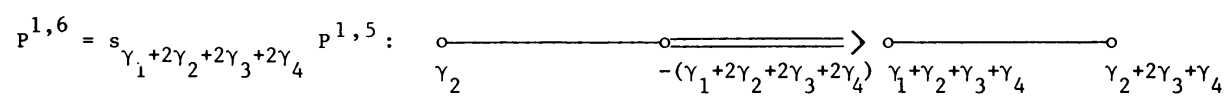

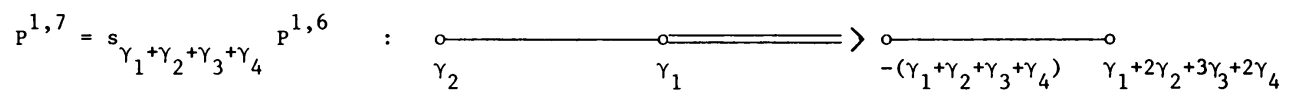

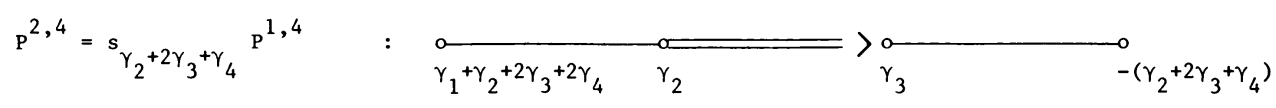

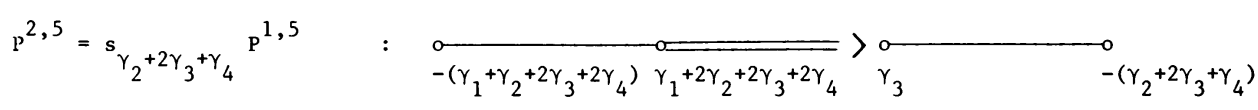

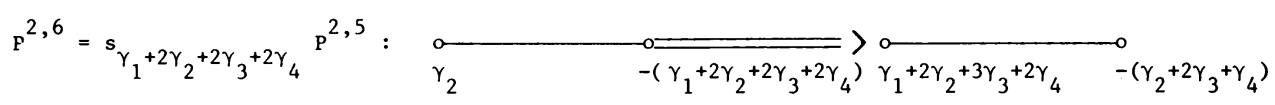

In the following we label $P^{i, j}$ by dots. We connect two dots with a line only if the corresponding systems of positive roots are related by a simple reflection:

\section{Diagram for $F_{4}$.}

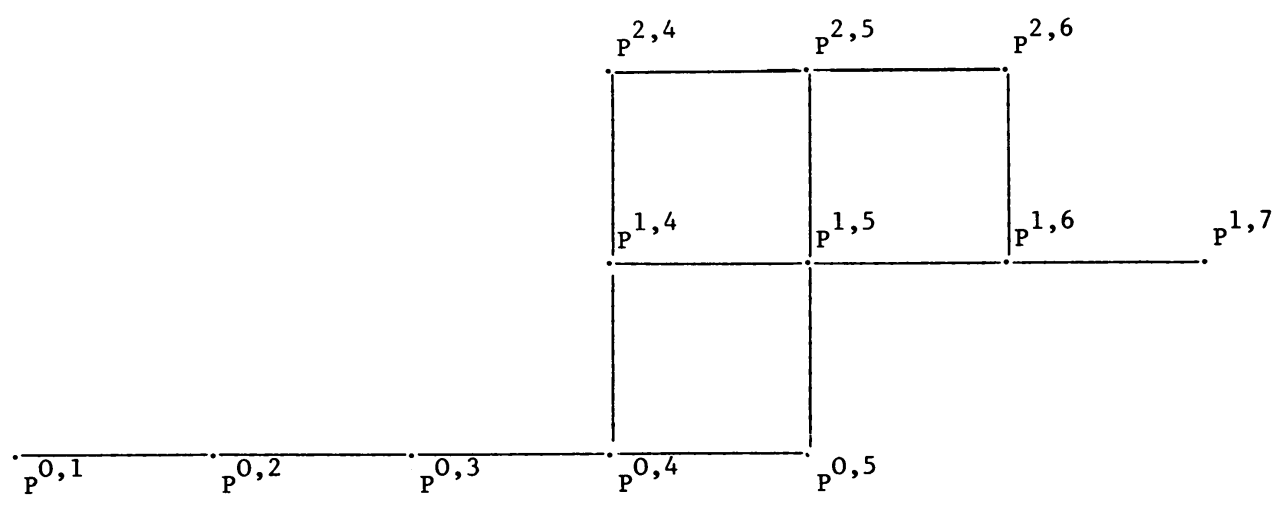


14. Some more computations. We now need to compute $\delta_{p i, j}$ for the systems of positive roots introduced in $\$ 13$. We recall that $2 \delta_{p i, j}=\Sigma_{\alpha \in P^{i}, \alpha}$.

LEMMA 14.1. (Spin $(2 n, 1))$

$$
2 \delta_{P, i}=(2 n-2 i+1) \tilde{\varepsilon}_{1}+\sum_{k=2}^{i}(2 n-2(k-1)+1) \tilde{\varepsilon}_{k}+\sum_{k=i+1}^{n}(2 n-2 k+1) \tilde{\varepsilon}_{k} .
$$

ProOF. $2 \delta_{P^{0, i}}=s_{\gamma_{i-1}^{i-1}} \cdots s_{\gamma_{1}} 2 \delta_{P^{0,1}}$.

$$
\begin{aligned}
& \text { Since } \gamma_{k}^{k}=\gamma_{1}+\cdots+\gamma_{k}=\tilde{\varepsilon}_{1}-\tilde{\varepsilon}_{k+1} \text { for } k=1, \ldots, n \text {, then } \\
& s_{\gamma_{i-1}^{i-1}} \cdots s_{\gamma_{1}^{1}} \tilde{\varepsilon}_{k}=\tilde{\varepsilon}_{k+1} \text { for } k=1, \ldots, i-1, \\
& s_{\gamma_{i-1}^{i-1}} \cdots s_{\gamma_{1}} \tilde{\varepsilon}_{i}=\tilde{\varepsilon}_{1} \text { and } \\
& s_{\gamma_{i-1}^{i-1}} \cdots s_{\gamma_{1}} \tilde{\varepsilon}_{k}=\tilde{\varepsilon}_{k} \quad \text { for } k \geqslant i+1 .
\end{aligned}
$$

Following Bourbaki [2]: $2 \delta_{P^{0,1}}=\sum_{k=1}^{n}(2 n-2 k+1) \tilde{\varepsilon}_{k}$ and hence

$$
\begin{aligned}
2 \delta_{P, i} & =\sum_{k=1}^{i-1}(2 n-2 k+1) \tilde{\varepsilon}_{k+1}+(2 n-2 i+1) \tilde{\varepsilon}_{1}+\sum_{k=i+1}^{n}(2 n-2 k+1) \tilde{\varepsilon}_{k} \\
& =(2 n-2 i+1) \tilde{\varepsilon}_{1}+\sum_{k=2}^{i}(2 n-2(k-1)+1) \tilde{\varepsilon}_{k}+\sum_{k=i+1}^{n}(2 n-2 k+1) \tilde{\varepsilon}_{k} .
\end{aligned}
$$

LEMMA 14.2. (SU $(n, 1))$

$$
\begin{aligned}
2 \delta_{p^{i j}}= & (n-2 i) \tilde{\varepsilon}_{1}+\sum_{k=2}^{i+1}(n-2(k-1)+2) \tilde{\varepsilon}_{k} \\
& +\sum_{k=i+2}^{n-j+1}(n-2 k+2) \tilde{\varepsilon}_{k} \\
& +\sum_{k=n-j+2}^{n}(n-2(k+1)+2) \tilde{\varepsilon}_{k}+(n-2(n-j+2)+2) \tilde{\varepsilon}_{n+1}
\end{aligned}
$$

Proof. Consider the permutation $\sigma$ defined by

$$
\begin{aligned}
\sigma\left(\tilde{\varepsilon}_{k}\right) & =\tilde{\varepsilon}_{k+1}, \quad k=1, \ldots, i, \\
\sigma\left(\tilde{\varepsilon}_{i+1}\right) & =\tilde{\varepsilon}_{1}, \\
\sigma\left(\tilde{\varepsilon}_{k}\right) & =\tilde{\varepsilon}_{k}, \quad k=i+2, \ldots, n-j+1, \\
\sigma\left(\tilde{\varepsilon}_{n-j+2}\right) & =\tilde{\varepsilon}_{n+1}, \\
\sigma\left(\tilde{\varepsilon}_{k}\right) & =\tilde{\varepsilon}_{k-1}, \quad k=n-j+3, \ldots, n+1 .
\end{aligned}
$$

Then $\sigma P^{0,1}=P^{i, j}, \quad 1 \leqslant i \leqslant n-1, \quad 1 \leqslant j \leqslant n-i$. Since $2 \delta_{P^{0,1}}=$ $\sum_{k=1}^{n+1}(n-2 k+2) \tilde{\varepsilon}_{k}$, then 


$$
\begin{aligned}
2 \delta_{P^{i j}}= & \sigma\left(\sum_{k=1}^{n+1}(n-2 k+2) \tilde{\varepsilon}_{k}\right)=(n-2 i) \tilde{\varepsilon}_{1}+\sum_{k=1}^{i}(n-2 k+2) \tilde{\varepsilon}_{k+1} \\
& +\sum_{k=i+2}^{n-j+1}(n-2 k+2) \tilde{\varepsilon}_{k}+(n-2(n-j+2)+2) \tilde{\varepsilon}_{n+1} \\
& +\sum_{k=n-j+3}^{n+1}(n-2 k+2) \tilde{\varepsilon}_{k-1} \\
= & (n-2 i) \tilde{\varepsilon}_{1}+\sum_{k=2}^{i+1}(n-2(k-1)+2) \tilde{\varepsilon}_{k}+\sum_{k=i+2}^{n-j+1}(n-2 k+2) \tilde{\varepsilon}_{k} \\
& +\sum_{k=n-j+2}^{n}(n-2(k+1)+2) \tilde{\varepsilon}_{k}+(n-2(n-j+2)+2) \tilde{\varepsilon}_{n+1} .
\end{aligned}
$$

LEMMA 14.3. $(\operatorname{Sp}(n, 1))$

$$
\begin{aligned}
2 \delta_{P^{i j}}=2\left\{(n+1-i) \tilde{\varepsilon}_{1}+(n+1-j) \tilde{\varepsilon}_{2}+\sum_{k=3}^{i+2}(n+1-(k-2)+1) \tilde{\varepsilon}_{k}\right. \\
\left.+\sum_{k=i+3}^{j+1}(n+1-(k-1)+1) \tilde{\varepsilon}_{k}+\sum_{k=j+2}^{n+1}(n+1-k+1) \tilde{\varepsilon}_{k}\right\} \\
2 \delta_{P^{i j+n}}=2\left\{(n+1-i) \tilde{\varepsilon}_{1}-j \tilde{\varepsilon}_{2}+\sum_{k=3}^{i+2}(n+1-(k-2)+1) \tilde{\varepsilon}_{k}\right. \\
\left.\quad+\sum_{k=i+3}^{n+2-j}(n+1-(k-1)+1) \tilde{\varepsilon}_{k}+\sum_{n+3-j}^{n+1}(n+1-k+1) \tilde{\varepsilon}_{k}\right\} .
\end{aligned}
$$

Proof. Let $\sigma$ be the permutation defined by $(0 \leqslant i<j<n)$

$$
\begin{array}{rlrl}
\sigma\left(\tilde{\varepsilon}_{k}\right) & =\tilde{\varepsilon}_{k+2}, & & k=1, \ldots, i, \\
\sigma\left(\tilde{\varepsilon}_{i+1}\right) & =\tilde{\varepsilon}_{1}, & \\
\sigma\left(\tilde{\varepsilon}_{k}\right) & =\tilde{\varepsilon}_{k+1}, & & k=i+2, \ldots, j, \\
\sigma\left(\tilde{\varepsilon}_{j+1}\right) & =\tilde{\varepsilon}_{2}, \\
\sigma\left(\tilde{\varepsilon}_{k}\right) & =\tilde{\varepsilon}_{k}, \quad k=j+2, \ldots, n+1 .
\end{array}
$$

Then $\sigma P^{0,1}=P^{i, j}$. By Bourbaki [2]

$$
2 \delta_{P^{0,1}}=2 \sum_{j=1}^{n+1}(n+1-j+1) \tilde{\varepsilon}_{j}
$$


and hence

$$
\begin{aligned}
& 2 \delta_{P^{i j}}=\sigma\left(2 \delta_{P^{0,1}}\right)=2\left\{\sigma \left(\sum_{k=1}^{i}(n+1-k+1) \tilde{\varepsilon}_{k}+(n+1-i-1+1) \tilde{\varepsilon}_{i+1}\right.\right. \\
& +\sum_{k=i+2}^{j}(n+1-k+1) \tilde{\varepsilon}_{j}+(n+1-j-1+1) \tilde{\varepsilon}_{j+1} \\
& \left.\left.+\sum_{k=j+2}^{n+1}(n+1-k+1) \tilde{\varepsilon}_{k}\right)\right\} \\
& =2\left\{\sum_{k=1}^{i}(n+1-k+1) \tilde{\varepsilon}_{k+2}+(n+1-i) \tilde{\varepsilon}_{1}\right. \\
& +\sum_{k=i+2}^{j}(n+1-k+1) \tilde{\varepsilon}_{k+1}+(n+1-j) \tilde{\varepsilon}_{2} \\
& \left.+\sum_{k=j+2}^{n+1}(n+1-k+1) \tilde{\varepsilon}_{k}\right\} \\
& =2\left\{\sum_{k=3}^{i+2}(n+1-(k-2)+1) \varepsilon_{k}+(n+1-i) \tilde{\varepsilon}_{1}\right. \\
& +\sum_{k=i+3}^{j+1}(n+1-(k-1)+1) \tilde{\varepsilon}_{k} \\
& \left.+(n+1-j) \tilde{\varepsilon}_{2}+\sum_{k=j+2}^{n+1}(n+1-k+1) \tilde{\varepsilon}_{k}\right\} \\
& =2\left\{(n+1-i) \tilde{\varepsilon}_{1}+(n+1-j) \tilde{\varepsilon}_{2}+\sum_{k=3}^{i+2}(n+1-(k-2)+1) \tilde{\varepsilon}_{k}\right. \\
& \left.+\sum_{k=i+3}^{j+1}(n+1-(k-1)+1) \tilde{\varepsilon}_{k}+\sum_{k=j+2}^{n+1}(n+1-k+1) \tilde{\varepsilon}_{k}\right\} .
\end{aligned}
$$

Let $\sigma_{1}$ be the element in the Weyl group, $W\left(\alpha_{1}, \ldots, \alpha_{n+1}\right)$, defined by

$$
\begin{gathered}
\sigma_{1}\left(\tilde{\varepsilon}_{k}\right)=\tilde{\varepsilon}_{k+2}, \quad k=1, \ldots, i, \\
\sigma_{1}\left(\tilde{\varepsilon}_{i+1}\right)=\tilde{\varepsilon}_{1}, \\
\sigma_{1}\left(\tilde{\varepsilon}_{k}\right)=\tilde{\varepsilon}_{k+1}, \quad k=i+2, \ldots, n+1-j, \\
\sigma_{1}\left(\tilde{\varepsilon}_{n+2-j}\right)=-\tilde{\varepsilon}_{2}, \\
\sigma_{1}\left(\tilde{\varepsilon}_{k}\right)=\tilde{\varepsilon}_{k}, \quad k=n+3-j, \ldots, n+1,
\end{gathered}
$$


then $\sigma_{1} P^{0,1}=P^{i, j+n}$ and hence

$$
\begin{aligned}
2 \delta_{P^{i j+n}}=\sigma_{1}\left(2 \delta_{P^{0,1}}\right)=2\{(n+1 & -i) \tilde{\varepsilon}_{1}-(n+1-(n+2-j)+1) \tilde{\varepsilon}_{2} \\
& +\sum_{k=3}^{i+2}(n+1-(k-2)+1) \tilde{\varepsilon}_{k} \\
& +\sum_{k=i+3}^{n+2-j}(n+1-(k-1)+1) \tilde{\varepsilon}_{k} \\
& \left.+\sum_{n+3-j}^{n+1}(n+1-k+1) \tilde{\varepsilon}_{k}\right\} .
\end{aligned}
$$

LEMMA 14.4. $\left(F_{4}\right)$

$$
\begin{array}{lc}
2 \delta_{P^{0,1}}=(11,5,3,1), & 2 \delta_{P^{1,5}}=(4,10,6,2), \\
2 \delta_{P^{0,2}}=(10,6,4,2), & 2 \delta_{P^{1,6}}=(2,10,6,4), \\
2 \delta_{P^{0,3}}=(9,7,5,1), & 2 \delta_{P^{1,7}}=(1,9,7,5), \\
2 \delta_{P^{0,4}}=(7,9,5,1), & 2 \delta_{P^{2,4}}=(5,11,3,1), \\
2 \delta_{P^{0,5}}=(5,9,7,1) & 2 \delta_{P^{2,5}}=(3,11,5,1), \\
2 \delta_{P^{1,4}}=(6,10,4,2), & 2 \delta_{P^{2,6}}=(1,11,5,3) .
\end{array}
$$

Proof. Following [2] the computation is straightforward.

15. $M$-types which give trivial infinitesimal character. We recall that $2 \delta_{m}=$ $\Sigma_{\alpha \in \Phi_{m}^{+}} \alpha$.

LEMMA 15.1. $G=\operatorname{Spin}(2 n, 1)$. Define $\Lambda_{0, i}=\delta_{P^{0, i}}-\delta_{m}$ and $\nu_{0, i}=2\left(\delta_{P^{0, i}, \alpha}\right) /(\alpha, \alpha)$ for $i=1, \ldots, n$. Then

(1) $\Lambda_{0, i}=\left(n-i+\frac{1}{2}\right) \tilde{\varepsilon}_{1}+\sum_{k=2}^{i} \tilde{\varepsilon}_{k}$,

(2) $\nu_{0, i}=2\left(n-i+\frac{1}{2}\right)$.

Proof. Following Bourbaki [2],

$$
\begin{aligned}
2 \delta_{m} & =\sum_{k=1}^{n-1} k(2(n-1)-k) \gamma_{k+1}=\sum_{k=2}^{n}(k-1)(2(n-1)-k+1) \gamma_{k} \\
& =(2(n-1)-1) \tilde{\varepsilon}_{2}+\sum_{k=3}^{n-1}(2 n-2 k+1) \tilde{\varepsilon}_{k}+\left((n-1)^{2}-n(n-2)\right) \tilde{\varepsilon}_{n} \\
& =\sum_{k=2}^{n}(2 n-2 k+1) \tilde{\varepsilon}_{k} .
\end{aligned}
$$

Thus,

$$
\begin{aligned}
2 \delta_{P 0, i}-2 \delta_{m}= & (2 n-2 i+1) \tilde{\varepsilon}_{1}+\sum_{k=2}^{i}(2 n-2(k-1)+1) \tilde{\varepsilon}_{k} \\
& +\sum_{k=i+1}^{n}(2 n-2 k+1) \tilde{\varepsilon}_{k}-\sum_{k=2}^{n}(2 n-2 k+1) \tilde{\varepsilon}_{k} \\
= & (2 n-2 i+1) \tilde{\varepsilon}_{1}+\sum_{k=2}^{i} 2 \tilde{\varepsilon}_{k}
\end{aligned}
$$

and (1) follows. 
Since $\alpha=\tilde{\varepsilon}_{1},(2)$ is evident.

LEMMA 15.2. $G=\operatorname{SU}(n, 1)$. Set $\Lambda_{i, j}=\delta_{p^{i j}}-\delta_{m}$ and $\nu_{i, j}=2\left(\delta_{p^{i j}}, \alpha\right) /(\alpha, \alpha)$ for $0 \leqslant i \leqslant n-1$ and $1 \leqslant j \leqslant n-i$. Then

(1) $\Lambda_{i, j}=\frac{1}{2}(n-2 i) \tilde{\varepsilon}_{1}+\sum_{k=2}^{i+1} \tilde{\varepsilon}_{k}-\sum_{k=n+2-j}^{n} \tilde{\varepsilon}_{k}+\frac{1}{2}(n-2(n-j+2)+2) \tilde{\varepsilon}_{n+1}$,

(2) $\nu_{i, j}=n-j-i+1$.

Proof. Following Bourbaki [2],

$$
\begin{aligned}
2 \delta_{m} & =\sum_{j=1}^{n-2} j(n-2-j+1) \gamma_{j+1}=\sum_{j=2}^{n-1}(j-1)(n-j) \gamma_{j} \\
& =(n-2) \tilde{\varepsilon}_{2}+\sum_{j=3}^{n-1}(n-2 j+2) \tilde{\varepsilon}_{j}-(n-2) \tilde{\varepsilon}_{n}=\sum_{j=2}^{n}(n-2 j+2) \tilde{\varepsilon}_{j} .
\end{aligned}
$$

Hence

$$
\begin{aligned}
2 \delta_{P^{i j}}-2 \delta_{m} & =(n-2 i) \tilde{\varepsilon}_{1}+\sum_{k=2}^{i+1}(n-2(k-1)+2) \tilde{\varepsilon}_{k}+\sum_{k=i+2}^{n-j+1}(n-2 k+2) \tilde{\varepsilon}_{k} \\
& +\sum_{k=n-j+2}^{n}(n-2(k+1)+2) \tilde{\varepsilon}_{k}+(n-2(n-j+2)+2) \tilde{\varepsilon}_{n+1} \\
& -\sum_{j=2}^{n}(n-2 j+2) \tilde{\varepsilon}_{j} \\
= & (n-2 i) \tilde{\varepsilon}_{1}+\sum_{k=2}^{i+1} 2 \tilde{\varepsilon}_{k}+\sum_{k=n-j+2}^{n}(-2) \tilde{\varepsilon}_{k}+(n-2(n-j+2)+2) \tilde{\varepsilon}_{n+1}
\end{aligned}
$$

and (1) follows. Since $\alpha=\tilde{\varepsilon}_{1}-\tilde{\varepsilon}_{n+1}$, (2) is immediate.

LemMA 15.3. $G=\operatorname{Sp}(n, 1)$. Set $\Lambda_{i, j}=\delta_{p^{i j}}-\delta_{m}, \nu_{i j}=2\left(\delta_{P^{i j}}, \alpha\right) /(\alpha, \alpha)$ for $0<i$ $<j \leqslant n$ and $\Lambda_{i j+n}=\delta_{P^{i j+n}}-\delta_{m}, \nu_{i j+n}=2\left(\delta_{P^{i j+n}}, \alpha\right) /(\alpha, \alpha)$ for $1<i+j<n$. Then

$$
\begin{aligned}
\Lambda_{i, j} & =\left(n+\frac{1}{2}-i\right) \tilde{\varepsilon}_{1}+\left(n+\frac{3}{2}-j\right) \tilde{\varepsilon}_{2}+\sum_{k=3}^{i+2} 2 \tilde{\varepsilon}_{k}+\sum_{k=i+3}^{j+1} \tilde{\varepsilon}_{k}, \\
\nu_{i, j} & =2 n-i-j+2
\end{aligned}
$$

and

$$
\begin{aligned}
\Lambda_{i, j+n} & =\left(n-i+\frac{1}{2}\right) \tilde{\varepsilon}_{1}+\left(\frac{1}{2}-j\right) \tilde{\varepsilon}_{2}+\sum_{k=3}^{i+2} 2 \tilde{\varepsilon}_{k}+\sum_{k=i+3}^{n+2-j} \tilde{\varepsilon}_{k}, \\
\nu_{i, j+n} & =n+1-i-j .
\end{aligned}
$$


340

M. W. BALDONI SILVA

Proof.

$$
\begin{aligned}
2 \delta_{m}= & \sum_{j=1}^{n-2} j(2(n-1)-j+1) \gamma_{j+2}+(n-1) n / 2 \gamma_{n+1}+\gamma_{1} \\
= & \sum_{j=3}^{n}(j-2)(2(n-1)-j+3) \gamma_{j}+(n-1) n \tilde{\varepsilon}_{n+1}+\tilde{\varepsilon}_{1}-\tilde{\varepsilon}_{2} \\
= & \sum_{j=3}^{n}(j-2)(2 n-j+1) \gamma_{j}+(n-1) n \tilde{\varepsilon}_{n+1}+\tilde{\varepsilon}_{1}-\tilde{\varepsilon}_{2} \\
= & (3-2)(2 n-3+1) \tilde{\varepsilon}_{3} \\
& +\sum_{j=4}^{n}\{(j-2)(2 n-j+1)-(j-3)(2 n-j+2)\} \tilde{\varepsilon}_{j} \\
& -(n-2)(n+1) \tilde{\varepsilon}_{n+1}+(n-1) n \tilde{\varepsilon}_{n+1}+\tilde{\varepsilon}_{1}-\tilde{\varepsilon}_{2} \\
= & (2 n-2) \tilde{\varepsilon}_{3}+\sum_{j=4}^{n}(2 n-2 j+4) \tilde{\varepsilon}_{j}+2 \tilde{\varepsilon}_{n+1}+\tilde{\varepsilon}_{1}-\tilde{\varepsilon}_{2} \\
= & \tilde{\varepsilon}_{1}-\tilde{\varepsilon}_{2}+\sum_{j=3}^{n+1} 2(n-j+2) \tilde{\varepsilon}_{j} .
\end{aligned}
$$

Hence

$$
\begin{aligned}
2 \delta_{P^{i j}}-2 \delta_{m}=2\{( & +1-i) \tilde{\varepsilon}_{1}+(n+1-j) \tilde{\varepsilon}_{2}+\sum_{k=3}^{i+2}(n+1-(k-2)+1) \tilde{\varepsilon}_{k} \\
& +\sum_{k=i+3}^{j+1}(n+1-(k-1)+1) \tilde{\varepsilon}_{k} \\
& \left.+\sum_{k=j+2}^{n+1}(n+1-k+1) \tilde{\varepsilon}_{k}-\frac{1}{2} \tilde{\varepsilon}_{1}+\frac{1}{2} \tilde{\varepsilon}_{2}-\sum_{j=3}^{n+1}(n-j+2) \tilde{\varepsilon}_{j}\right\} \\
= & 2\left\{\left(n-i+\frac{1}{2}\right) \tilde{\varepsilon}_{1}+\left(n-j+\frac{3}{2}\right) \tilde{\varepsilon}_{2}+\sum_{k=3}^{i+2} 2 \tilde{\varepsilon}_{k}+\sum_{k=i+3}^{j+1} \tilde{\varepsilon}_{k}\right\}
\end{aligned}
$$

and (1) follows since $\alpha=\tilde{\varepsilon}_{1}+\tilde{\varepsilon}_{2}$.

We now prove (2).

$$
\begin{aligned}
& 2 \delta_{p^{i j+n}}-2 \delta_{m}=2\{(+1-i) \tilde{\varepsilon}_{1}-j \tilde{\varepsilon}_{2}+\sum_{k=3}^{i+2}(n+1-(k-2)+1) \tilde{\varepsilon}_{k} \\
& \quad+\sum_{k=i+3}^{n+2-j}(n+1-(k-1)+1) \tilde{\varepsilon}_{k} \\
&\left.+\sum_{n+3-j}^{n+1}(n+1-k+1) \tilde{\varepsilon}_{k}-\frac{1}{2} \tilde{\varepsilon}_{1}+\frac{1}{2} \tilde{\varepsilon}_{2}-\sum_{j=3}^{n+1}(n-j+2) \tilde{\varepsilon}_{j}\right\} \\
&=2\left\{\left(n-i+\frac{1}{2}\right) \tilde{\varepsilon}_{1}-\left(j-\frac{1}{2}\right) \tilde{\varepsilon}_{2}+\sum_{k=3}^{i+2} 2 \tilde{\varepsilon}_{k}+\sum_{k=i+3}^{n+2-j} \tilde{\varepsilon}_{k}\right\} .
\end{aligned}
$$


The expression for $\nu_{i, j+n}$ is clear, so the proof is complete.

LEMMA 15.4. $G=F_{4}$. Set $\Lambda_{i, j}=\delta_{p^{i j}}-\delta_{m}$ and $\nu_{i, j}=2\left(\delta_{P^{i j}}, \alpha\right) /(\alpha, \alpha)$. Then:

(1) $\Lambda_{0,1}=\frac{1}{2}(11,0,0,0), \nu_{0,1}=11$,

(2) $\Lambda_{0,2}=\frac{1}{2}(10,1,1,1), \nu_{0,2}=10$,

(3) $\Lambda_{0,3}=\frac{1}{2}(9,2,2,0), \nu_{0,3}=9$,

(4) $\Lambda_{0,4}=\frac{1}{2}(7,4,2,0), \nu_{0,4}=7$,

(5) $\Lambda_{0,5}=\frac{1}{2}(5,4,4,0), \nu_{0,5}=5$,

(6) $\Lambda_{1,4}=\frac{1}{2}(6,5,1,1), \nu_{1,4}=6$,

(7) $\Lambda_{1,5}=\frac{1}{2}(4,5,3,1), \nu_{1,5}=4$,

(8) $\Lambda_{1,6}=\frac{1}{2}(2,5,3,3), \nu_{1,6}=2$,

(9) $\Lambda_{1,7}=\frac{1}{2}(1,4,4,4), \nu_{1,7}=1$,

(10) $\Lambda_{2,4}=\frac{1}{2}(5,6,0,0), \nu_{2,4}=5$,

(11) $\Lambda_{2,5}=\frac{1}{2}(3,6,2,0), \nu_{2,5}=3$,

(12) $\Lambda_{2,6}=\frac{1}{2}(1,6,2,2), \nu_{2,6}=1$.

Proof. Since $2 \delta_{m}=5 \tilde{\varepsilon}_{2}+3 \tilde{\varepsilon}_{3}+\tilde{\varepsilon}_{4}$ and $\alpha=\tilde{\varepsilon}_{1}$, using 14.4 , the computation is straightforward.

15.5. Let $\pi_{\mu}$ be one of the $M$-representation we found in $\S 12$. We have computed its highest $\mu$ weight with respect to the $\varepsilon_{i}^{\prime}$ 's. A moment's thought shows, how, in each case, the highest weight $\mu$ of $\pi_{\mu}$ reads with respect to the $\tilde{\varepsilon}_{i}$ 's.

As we remarked in 11.1, we now use the equation of the infinitesimal character, to see which of these $M$-types will eventually give an embedding.

LeMMA 15.6. (Spin $(2 n, 1))$

$$
\mu_{1}=\sum_{i=2}^{n} \tilde{\varepsilon}_{i}=\Lambda_{0,\left.n\right|_{b_{b}}} .
$$

ProOF. $\Lambda_{0, n}=\frac{1}{2} \tilde{\varepsilon}_{1}+\sum_{k=2}^{n} \tilde{\varepsilon}_{k}$, hence $\Lambda_{0,\left.n\right|_{\mathfrak{Y}^{-}}}=\sum_{k=2}^{n} \tilde{\varepsilon}_{k}$.

LEMMA 15.7. ( $\mathrm{SU}(n, 1))$

(1) $\mu_{1}^{i}=\Lambda_{i, n-\left.i\right|_{\mathfrak{b}}}$ for $i=0, \ldots, n-1$,

(2) $\mu_{2}^{i}=\Lambda_{i-1, n-\left.i\right|_{\mathfrak{b}}}$ for $i=1, \ldots, n-1$,

(3) $\mu_{3}^{i}=\Lambda_{i-1, n-i+\left.1\right|_{b_{-}}}$for $i=1, \ldots, n$.

ProOF.

$$
\begin{aligned}
\Lambda_{i,\left.j\right|_{b}-}= & \frac{1}{2}(n-2 i+n-2(n-j+2)+2) \frac{\left(\tilde{\varepsilon}_{1}+\tilde{\varepsilon}_{n+1}\right)}{2} \\
& +\sum_{k=2}^{i+1} \tilde{\varepsilon}_{k}-\sum_{k=n+2-j}^{n} \tilde{\varepsilon}_{k} \\
= & \left(\frac{j-i-1}{2}\right)\left(\tilde{\varepsilon}_{1}+\tilde{\varepsilon}_{n+1}\right)+\sum_{k=2}^{i+1} \tilde{\varepsilon}_{k}-\sum_{k=n+2-j}^{n} \tilde{\varepsilon}_{k} .
\end{aligned}
$$


Hence

$$
\begin{aligned}
& \Lambda_{i, n-\left.i\right|_{\mathfrak{h}^{-}}}=\frac{n-2 i-1}{2}\left(\tilde{\varepsilon}_{1}+\tilde{\varepsilon}_{n+1}\right)+\sum_{k=2}^{i+1} \tilde{\varepsilon}_{k}-\sum_{k=i+2}^{n} \tilde{\varepsilon}_{k}=\mu_{1}^{i} \quad i=0, \ldots, n-1 . \\
& \Lambda_{i-1, n-\left.i\right|_{\mathfrak{b}^{-}}}=\frac{n-2 i}{2}\left(\tilde{\varepsilon}_{1}+\tilde{\varepsilon}_{n+1}\right)+\sum_{k=2}^{i} \tilde{\varepsilon}_{k}-\sum_{k=i+2}^{n} \tilde{\varepsilon}_{k}=\mu_{2}^{i} \quad i=1, \ldots, n-1 . \\
& \Lambda_{i-1, n-i+\left.1\right|_{\mathfrak{b}^{-}}}=\frac{n-2 i+1}{2}\left(\tilde{\varepsilon}_{1}+\tilde{\varepsilon}_{n+1}\right)+\sum_{k=2}^{i} \tilde{\varepsilon}_{k}-\sum_{k=i+1}^{n} \varepsilon_{k}=\mu_{3}^{i} \quad i=1, \ldots, n .
\end{aligned}
$$

LEMMA 15.8. ( $\mathrm{Sp}(n, 1))$ We have the following table, where "NO" means that the particular M-type does not give trivial infinitesimal character, i.e. is different from $\Lambda_{i,\left.\right|_{b}-}$ or $\Lambda_{i, j+\left.n\right|_{b^{-}}}$, for all possible values of $i$ and $j$. Otherwise, we give the corresponding $\Lambda_{i, j}$.

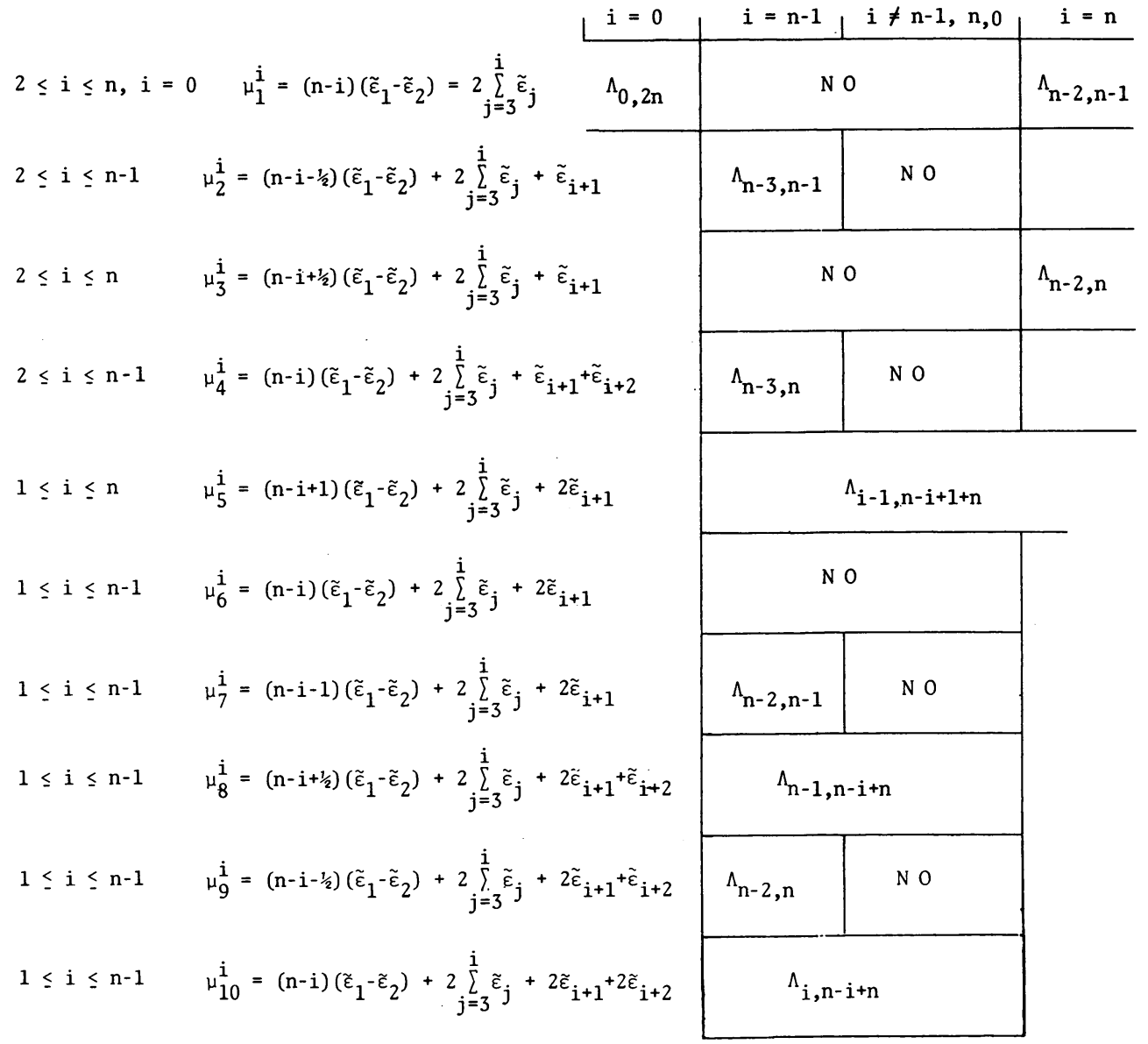


Proof.

$$
\Lambda_{k, l l_{\left.\right|^{-}}}=\frac{-k+l-1}{2}\left(\tilde{\varepsilon}_{1}-\tilde{\varepsilon}_{2}\right)+2 \sum_{t=3}^{k+2} \tilde{\varepsilon}_{t}+\sum_{t=k+3}^{l+1} \tilde{\varepsilon}_{t},
$$

where

$$
\sum_{3}^{k+2} \tilde{\varepsilon}_{t}=0 \Leftrightarrow k=0 \text { and } \sum_{k+3}^{l+1} \tilde{\varepsilon}_{t}=0 \Leftrightarrow l=k+1,
$$

$(0 \leqslant k<l \leqslant n)$. Similarly,

$$
\Lambda_{k, l+n_{\mid b^{-}}}=\frac{n-k+l}{2}\left(\tilde{\varepsilon}_{1}-\tilde{\varepsilon}_{2}\right)+2 \sum_{t=3}^{k+2} \tilde{\varepsilon}_{t}+\sum_{t=k+3}^{n+2-l} \tilde{\varepsilon}_{t},
$$

where

$$
\sum_{t=3}^{k+2} \tilde{\varepsilon}_{t}=0 \Leftrightarrow k=0 \text { and } \sum_{t=k+3}^{n+2-l} \tilde{\varepsilon}_{t}=0 \Leftrightarrow l=n-k,
$$

$(1 \leqslant k+l \leqslant n)$. We also note that if $2 \leqslant i$, then

$$
\sum_{j=3}^{i} \tilde{\varepsilon}_{j}=\sum_{j=3}^{k+2} \tilde{\varepsilon}_{j} \Leftrightarrow k+2=i .
$$

For convenience in what follows we write $\Lambda_{k, l}$ for $\Lambda_{k,\left.l\right|_{b}-}$. Since $\tilde{\varepsilon}_{1}-\tilde{\varepsilon}_{2}$, $\tilde{\varepsilon}_{3}, \ldots, \tilde{\varepsilon}_{n+1}$ are linearly independent on $\mathfrak{h}^{-}$, then

$2 \leq i \leq n, \mu_{1}^{i}=\Lambda_{k, \ell} \Leftrightarrow\left\{\begin{array}{l}n-i=\frac{-k+\ell-1}{2} \\ k+2=i \\ \ell=k+1\end{array} \Leftrightarrow\left\{\begin{array}{l}n-i=0 \\ k+2=i \\ \ell=k+1\end{array} \Leftrightarrow\left\{\begin{array}{l}n=i \\ k=n-2 \\ \ell=n-1\end{array}\right.\right.\right.$

$2 \leq i \leq n, \quad \mu_{1}^{i}=\Lambda_{k, \ell+n} \Leftrightarrow\left\{\begin{array}{l}n-i=\frac{n-k+\ell}{2} \\ k+2=i \\ \ell=n-k\end{array} \Longleftrightarrow\left\{\begin{array}{l}n-i=n-k \\ k=i-2 \\ l=n-k\end{array}\right.\right.$ which is impossible $\forall \ell, k$.

$\mu_{1}^{0}=\Lambda_{k, \ell} \Leftrightarrow\left\{\begin{array}{l}\mathrm{n}=\frac{-\mathrm{k}+\ell-1}{2} \\ \mathrm{k}=0 \\ \ell=1\end{array} \Longleftrightarrow\left\{\begin{array}{l}\mathrm{n}=0 \\ \mathrm{k}=0 \\ \ell=0\end{array} \quad\right.\right.$ which is impossible.

$\mu_{1}^{0}=\Lambda_{\mathrm{k}, \ell+\mathrm{n}} \Longleftrightarrow\left\{\begin{array}{l}\mathrm{n}=\frac{\mathrm{n}-\mathrm{k}+\ell}{2} \\ \mathrm{k}=0 \\ \ell=\mathrm{n}\end{array} \Longleftrightarrow\left\{\begin{array}{l}\mathrm{k}=0 \\ \ell=\mathrm{n}\end{array}\right.\right.$

$\mu_{2}^{i}=\Lambda_{k, \ell} \Longleftrightarrow\left\{\begin{array}{l}n-i-\frac{1}{2}=\frac{-k+l-1}{2} \\ k+2=i \\ k+3=\ell+1\end{array} \Longleftrightarrow\left\{\begin{array}{l}n-i-\frac{1}{2}=\frac{-k+\ell-1}{2} \\ k+2=i \\ k+2=\ell\end{array} \Longleftrightarrow\left\{\begin{array}{l}n-i=1 \\ i=l \\ k=i-2\end{array} \Longleftrightarrow\left\{\begin{array}{l}i=n-1 \\ l=n-1 \\ k=n-3\end{array}\right.\right.\right.\right.$

$\mu_{2}^{i}=\Lambda_{k, \ell+n} \Longleftrightarrow\left\{\begin{array}{l}n-i-\frac{1}{2}=\frac{n-k+\ell}{2} \\ k+2=i \\ n+2-\ell=k+3\end{array} \Longleftrightarrow\left\{\begin{array}{l}n-i-\frac{1}{2}=\frac{n-k+\ell}{2} \\ k+2=i \\ l=n-k-1\end{array} \Longleftrightarrow\left\{\begin{array}{l}i=k \\ k=i-2 \\ l=n-k-1\end{array} \quad\right.\right.\right.$ which is impossible $\forall k, l$. 


$$
\begin{aligned}
& \mu_{3}^{i}=\Lambda_{k, \ell} \Longleftrightarrow\left\{\begin{array} { l } 
{ n - i + \frac { 1 } { 2 } = \frac { - k + l - 1 } { 2 } } \\
{ k + 2 = i } \\
{ k + 3 = \ell + 1 }
\end{array} \Longleftrightarrow \left\{\begin{array} { l } 
{ n - i + \frac { 1 } { 2 } = \frac { 1 } { 2 } } \\
{ k + 2 = i } \\
{ k + 2 = \ell }
\end{array} \Longleftrightarrow \left\{\begin{array} { l } 
{ n = i } \\
{ k + 2 = i } \\
{ k + 2 = \ell }
\end{array} \Longleftrightarrow \left\{\begin{array}{l}
n=i \\
k=n-2 \\
l=n
\end{array}\right.\right.\right.\right. \\
& \mu_{3}^{i}=\Lambda_{k, \ell+n} \Leftrightarrow\left\{\begin{array} { l } 
{ n - i + \frac { 1 } { 2 } = \frac { n - k + \ell } { 2 } } \\
{ k + 2 = i } \\
{ \ell = n - k - 1 }
\end{array} \Longleftrightarrow \left\{\begin{array} { l } 
{ n - i + \frac { 1 } { 2 } = n - k - \frac { 1 } { 2 } } \\
{ k = i - 2 } \\
{ \ell = n - k - 1 }
\end{array} \Longleftrightarrow \left\{\begin{array} { l } 
{ i = k + 1 } \\
{ k = i - 2 } \\
{ l = n - k - 1 }
\end{array} \Longleftrightarrow \left\{\begin{array}{l}
k=i-1 \\
k=i-2 \\
\ell=n-k-1
\end{array}\right.\right.\right.\right. \\
& \text { which is impossible } \forall \mathrm{k}, \ell \text {. } \\
& \mu_{4}^{i}=\Lambda_{k, l} \Longleftrightarrow\left\{\begin{array} { l } 
{ \mathrm { n } - \mathrm { i } = \frac { - \mathrm { k } + \ell - 1 } { 2 } } \\
{ \mathrm { k } + 2 = \mathrm { i } } \\
{ \ell + 1 = \mathrm { k } + 4 }
\end{array} \Longleftrightarrow \left\{\begin{array} { l } 
{ \mathrm { n } - \mathrm { i } = \frac { 3 - 1 } { 2 } = 1 } \\
{ \mathrm { k } + 2 = \mathrm { i } } \\
{ \ell = \mathrm { k } + 3 }
\end{array} \Longleftrightarrow \left\{\begin{array}{l}
\mathrm{n}-1=\mathrm{i} \\
\mathrm{k}=\mathrm{n}-3 \\
\ell=\mathrm{n}
\end{array}\right.\right.\right. \\
& \mu_{4}^{i}=\Lambda_{k, \ell+n} \Longleftrightarrow\left\{\begin{array} { l } 
{ n - i = \frac { n - k + \ell } { 2 } } \\
{ k + 2 = i } \\
{ n + 2 - \ell = k + 4 }
\end{array} \Longleftrightarrow \left\{\begin{array} { l } 
{ n - i = \frac { n - k + l } { 2 } } \\
{ k = i - 2 } \\
{ n - k - 2 = \ell }
\end{array} \Longleftrightarrow \left\{\begin{array} { l } 
{ n - i = n - k - 1 } \\
{ k = i - 2 } \\
{ l = n - k - 2 }
\end{array} \Longleftrightarrow \left\{\begin{array}{l}
i=k+1 \\
k=i-2 \\
l=n-k-2
\end{array}\right.\right.\right.\right. \\
& \text { which is impossible } \forall \mathrm{k}, \ell \text {. } \\
& \mu_{5}^{i}=\Lambda_{k}, \ell \Leftrightarrow\left\{\begin{array} { l } 
{ n - i + 1 = \frac { - k + l - 1 } { 2 } } \\
{ k + 2 = i + 1 } \\
{ \ell = 1 + k }
\end{array} \Longleftrightarrow \left\{\begin{array} { l } 
{ n - i + 1 = 0 } \\
{ k = i - 1 } \\
{ \ell = k + 1 }
\end{array} \Leftrightarrow \left\{\begin{array}{l}
i=n+1 \\
k=i-1 \\
\ell=k+1
\end{array} \quad \text { which is impossible since } i \leq n-1 .\right.\right.\right. \\
& \mu_{5}^{i}=\Lambda_{k, \ell+n} \Longleftrightarrow\left\{\begin{array} { l } 
{ n - i + 1 = \frac { n - k + \ell } { 2 } } \\
{ k + 2 = i + 1 } \\
{ \ell = n - k }
\end{array} \Longleftrightarrow \left\{\begin{array} { l } 
{ n - i + 1 = n - k } \\
{ k = i - 1 } \\
{ \ell = n - k }
\end{array} \Longleftrightarrow \left\{\begin{array}{l}
k=i-1 \\
l=n-i+1
\end{array}\right.\right.\right. \\
& \mu_{6}^{i}=\Lambda_{k, \ell} \Longleftrightarrow\left\{\begin{array} { l } 
{ n - i = \frac { - k + \ell - 1 } { 2 } } \\
{ k + 2 = i + 1 } \\
{ \ell = k + 1 }
\end{array} \Longleftrightarrow \left\{\begin{array} { l } 
{ n - i = 0 } \\
{ k = i - 1 } \\
{ \ell = k + 1 }
\end{array} \Longleftrightarrow \left\{\begin{array}{l}
i=n \\
k=n-1 \\
l=n
\end{array} \quad \text { which is impossible since } i \leq n-1 .\right.\right.\right. \\
& \mu_{6}^{i}=\Lambda_{k, \ell+n} \Longleftrightarrow\left\{\begin{array} { l } 
{ n - i = \frac { n - k + \ell } { 2 } } \\
{ k + 2 = i + 1 } \\
{ \ell = n - k }
\end{array} \Longleftrightarrow \left\{\begin{array} { l } 
{ n - i = n - k } \\
{ k = i - 1 } \\
{ \ell = n - k }
\end{array} \Longleftrightarrow \left\{\begin{array}{l}
i=k \\
k=i-1 \\
\ell=n-k
\end{array} \text { which is impossible } \forall k, \ell .\right.\right.\right. \\
& \mu_{7}^{i}=\Lambda_{k, \ell} \Leftrightarrow\left\{\begin{array} { l } 
{ n - i - 1 = \frac { - k + \ell - 1 } { 2 } } \\
{ k + 2 = i + 1 } \\
{ \ell = k + 1 }
\end{array} \Longleftrightarrow \left\{\begin{array} { l } 
{ n - i - 1 = 0 } \\
{ k + 2 = i + 1 } \\
{ \ell = k + 1 }
\end{array} \Longleftrightarrow \left\{\begin{array}{l}
i=n-1 \\
k=n-2 \\
\ell=n-1
\end{array}\right.\right.\right. \\
& \mu_{7}^{i}=\Lambda_{k, \ell+n} \Longleftrightarrow\left\{\begin{array} { l } 
{ n - i - 1 = \frac { n - k + \ell } { 2 } } \\
{ k + 2 = i + 1 } \\
{ \ell = n - k }
\end{array} \Longleftrightarrow \left\{\begin{array} { l } 
{ n - i - 1 = n - k } \\
{ k = i - 1 } \\
{ \ell = n - k }
\end{array} \Longleftrightarrow \left\{\begin{array}{l}
k=i+1 \\
k=i-1 \\
\ell=n-k
\end{array} \quad \text { which is impossible } \forall k, \ell .\right.\right.\right.
\end{aligned}
$$


$\mu_{8}^{i}=\Lambda_{k, \ell} \Longleftrightarrow\left\{\begin{array}{l}n-i+\frac{1}{2}=\frac{-k+\ell-1}{2} \\ k+2=i+1 \\ \ell+1=k+3\end{array} \Longleftrightarrow\left\{\begin{array}{l}n-i+\frac{1}{2}=\frac{1}{2} \\ k+2=i+1 \\ \ell-k=2\end{array} \Longleftrightarrow\left\{\begin{array}{l}n=i \\ k=i-1 \\ \ell-k=2\end{array} \quad\right.\right.\right.$ which is impossible, since $i \leq n-1$.

$\mu_{8}^{i}=\Lambda_{k, \ell+n} \Leftrightarrow\left\{\begin{array}{l}n-i+\frac{1}{2}=\frac{n-k+\ell}{2} \\ k+2=i+1 \\ n+2-\ell=k+3\end{array} \Longleftrightarrow\left\{\begin{array}{l}n-i+\frac{1}{2}=n-k-\frac{1}{2} \\ k=i-1 \\ \ell=n-k-1\end{array} \Longleftrightarrow\left\{\begin{array}{l}k=i-1 \\ \ell=n-i\end{array}\right.\right.\right.$

$\mu_{9}^{i}=\Lambda_{k, l} \Leftrightarrow\left\{\begin{array}{l}\mathrm{n}-\mathrm{i}-\frac{1}{2}=\frac{-k+\ell-1}{2} \\ \mathrm{k}+2=\mathrm{i}+1 \\ \ell+1=\mathrm{k}+3\end{array} \Longleftrightarrow\left\{\begin{array}{l}\mathrm{n}-\mathrm{i}-\frac{1}{2}=\frac{1}{2} \\ \mathrm{k}=\mathrm{i}-1 \\ \ell=\mathrm{k}+2\end{array} \Longleftrightarrow\left\{\begin{array}{l}\mathrm{i}=\mathrm{n}-1 \\ \mathrm{k}=\mathrm{n}-2 \\ \ell=\mathrm{n}\end{array}\right.\right.\right.$

$u_{9}^{i}=\Lambda_{k, \ell+n} \Leftrightarrow\left\{\begin{array}{l}n-i-\frac{1}{2}=\frac{n-k+\ell}{2} \\ k+2=i+1 \\ n+2-l=k+3\end{array} \Leftrightarrow\left\{\begin{array}{l}n-i-\frac{1}{2}=n-k-\frac{1}{2} \\ k=i-1 \\ \ell=n-k-1\end{array} \Longleftrightarrow\left\{\begin{array}{l}i=k \\ k=i-1 \\ l=n-k-1\end{array}\right.\right.\right.$ which is impossible $\forall k, l$.

$\mu_{10}^{i}=\Lambda_{k, \ell} \Leftrightarrow\left\{\begin{array}{l}n-i=\frac{-k+\ell-1}{2} \\ k+2=i+2 \\ \ell=k+1\end{array} \Longleftrightarrow\left\{\begin{array}{l}n-i=0 \\ k=i \\ \ell=k+1\end{array}\right.\right.$ which is impossible since $i \leq n-1$.

$\mu_{10}^{i}=\Lambda_{k, \ell+n} \Longleftrightarrow\left\{\begin{array}{l}n-i=\frac{n-k+l}{2} \\ k+2=i+2 \\ \ell=n-k\end{array} \Longleftrightarrow\left\{\begin{array}{l}n-i=n-k \\ k=i \\ l=n-k\end{array} \Longleftrightarrow\left\{\begin{array}{l}i=k \\ l=n-i\end{array}\right.\right.\right.$.

Lemma 15.9. $\left(F_{4}\right)$ Let $\mu_{j}^{i}$ be as in Theorem 12.5, and $\Lambda_{i, j}$ as in 15.4. Then $\mu_{j}^{i}$ is equal to some $\Lambda_{i, j}$ if it is in the following list:

$$
\begin{array}{ll}
\Lambda_{0,1_{1 b^{-}}}=\mu_{18}^{2}, \mu_{5}^{3}, & \Lambda_{0,2_{\mid b^{-}}}=\mu_{13}^{2}, \mu_{4}^{3}, \\
\Lambda_{0,3_{1 b^{-}}}=\mu_{11}^{2}, & \Lambda_{0,4_{\mid b^{-}}}=\mu_{9}^{1}, \mu_{9}^{2}, \\
\Lambda_{0,5_{\mid b^{-}}}=\mu_{8}^{1}, \mu_{1}^{2}, & \Lambda_{1,4_{\mid b^{-}}}=\mu_{5}^{1}, \mu_{15}^{2}, \\
\Lambda_{1,5_{\mid b^{-}}}=\mu_{4}^{1}, \mu_{7}^{2}, & \Lambda_{1,6_{\mid b^{-}}}=\mu_{8}^{2}, \\
\Lambda_{1,7_{\mid b^{-}}}=\mu_{3}^{2}, \mu_{1}^{3}, & \Lambda_{2,6_{\mid b^{-}}}=\mu_{1}^{1}, \mu_{14}^{2} .
\end{array}
$$

Proof. If $\mu_{j}^{i}=\sum_{i=1}^{3} a_{i} \varepsilon_{i}$ is the highest weight of $\pi_{j}^{i}$, then the highest weight of $\pi_{j}^{i} \circ \phi$ with respect to

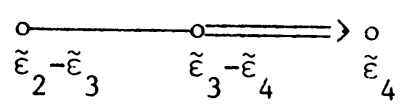

reads $\mu_{j}^{i}=\sum_{i=2}^{4} a_{i-1} \tilde{\varepsilon}_{i}$. Lemma 15.4 gives the expression for the $\Lambda_{i,\left.\right|_{b}-}$, as follows (for convenience write simply $\Lambda_{i, j}$ ):

$$
\begin{array}{cccc}
\Lambda_{0,1}=(0,0,0), & \Lambda_{0,2}=\left(\frac{1}{2}, \frac{1}{2}, \frac{1}{2}\right), & \Lambda_{0,3}=(1,1,0), & \Lambda_{0,4}=(2,1,0), \\
\Lambda_{0,5}=(2,2,0), & \Lambda_{1,4}=\left(\frac{5}{2}, \frac{1}{2}, \frac{1}{2}\right), & \Lambda_{1,5}=\left(\frac{5}{2}, \frac{3}{2}, \frac{1}{2}\right), & \Lambda_{1,6}=\left(\frac{5}{2}, \frac{3}{2}, \frac{3}{2}\right), \\
\Lambda_{1,7}=(2,2,2), & \Lambda_{2,4}=(3,0,0), & \Lambda_{2,5}=(3,1,0), & \Lambda_{2,6}=(3,1,1) .
\end{array}
$$


Comparing these expressions with the ones of the $\mu_{j}^{i}$ 's gives the result. Note that the only $M$-type which gives infinitesimal character zero and with multiplicity greater than one, precisely two, is $\mu_{13}^{2}$.

16. Some realizations of a discrete series representation. We generically fix a system of positive roots $P$ for $\Delta$. We want to prove that if $P$ contains two simple noncompact roots, then the discrete series $\pi_{\delta_{p}}$ is realized as a subspace in two distinct (nonequivalent) principal series. The result is a consequence of $[10$, Theorem 1.1].

Without loss of generality we may assume that $P \supset \Delta_{k}^{+}$.

LEMMA 16.1. If $P$ contains two simple noncompact roots, then $\pi_{\delta_{P}}$ is realized as a subspace in two distinct principal series.

Proof. Set, for convenience, $2 \delta_{n}^{P}=2 \delta_{n}, 2 \delta_{P}=2 \delta$ and $2 \delta_{k}^{\Delta_{k}^{+}}=2 \delta_{k}$. Let $\alpha_{1}, \alpha_{2}$ be two such simple roots. Choose $X_{ \pm \alpha_{i}} \in\left(g_{\mathbf{C}}\right)_{ \pm \alpha_{i}}(i=1,2)$ so that $X_{\alpha_{i}}+X_{-\alpha_{i}} \in \mathfrak{p}$. Let $a_{i}=\mathbf{R}\left(X_{\alpha_{i}}+X_{-\alpha_{i}}\right)$ and $m_{i}$ be the centralizer of $a_{i}$ in $k(i=1,2)$.

Let $\mathfrak{h}_{i}^{-}=\left\{H \in \mathfrak{h}, \alpha_{i}(H)=0\right\}(i=1,2)$; then $\mathfrak{h}_{i}^{-}$is a Cartan subalgebra of $\mathfrak{m}_{i}$. Let $u_{i}=\exp (\pi / 4)\left(X_{\alpha_{i}}-X_{-\alpha_{i}}\right)$ and let Ad $u_{i}$ be the Cayley transform.

Set $\Phi_{m_{i}}^{+}=\left\{\beta \circ \operatorname{Ad}\left(u_{i}\right)^{-1}, \beta \in \Delta^{+}\right.$and $\left.\left(\beta, \alpha_{i}\right)=0\right\}$, then $\Phi_{m_{i}}^{+}$is a system of positive roots for $\left(\mathfrak{m}_{i},\left(\mathfrak{h}_{i}^{-}\right)_{\mathbf{C}}\right)$. Let $M_{i}$ as usual be the centralizer of $a_{i}$ in $K$. Form restricted roots $\Lambda_{i}$ with respect to $a_{i}$, and let $\Lambda_{i}^{+}=\left\{\lambda \in \Lambda_{i}, \lambda\left(X_{\alpha_{i}}+X_{\alpha_{i}}\right)>0\right\}$; finally, let $\rho_{i}$ and $n_{i}$ be defined accordingly to $\Lambda_{i}^{+}$. If $\xi_{i}(i=1,2)$ is the $M_{i}$-representation of highest weight $2 \delta_{\left.n\right|_{\bar{i}}} \Lambda_{\xi_{i}}$, relative to $\Phi_{m_{i}}^{+}$, then Knapp and Wallach [10] prove that $\pi_{\delta}$ is infinitesimally equivalent to a subspace of $\pi_{\xi_{i}, \nu_{i}}$, where $\nu_{i} \in a_{i}^{*}$ is defined by

$$
\nu_{i}\left(X_{\alpha_{i}}+X_{-\alpha_{i}}\right)=-\frac{2\left(\delta, \alpha_{i}\right)}{\left(\alpha_{i}, \alpha_{i}\right)}=1 .
$$

Hence to prove the lemma, we need to show that these two principal series are not equivalent.

Set $\beta=\alpha_{1}+\alpha_{2}$, then, due to the results of $\S 11, \beta$ is a simple compact root.

Since in rank one all the noncompact roots have the same length (cf. $[10,12.1])$, it is clear that $s_{\beta} \alpha_{1}=-\alpha_{2}$ and $s_{\beta} \alpha_{2}=-\alpha_{1}$.

Let $k=\exp \left[\pi / \sqrt{2}(\beta, \beta)^{1 / 2}\right]\left(X_{\beta}-X_{-\beta}\right)$, where $X_{\beta}$ is chosen so that $\left[X_{\beta}, X_{-\alpha_{1}}\right]$ $=(\beta, \beta)^{1 / 2} X_{\alpha_{2}} / \sqrt{2}$. Then $\operatorname{Ad}(k)\left(X_{\alpha_{1}}+X_{-\alpha_{1}}\right)=\cos \pi / 2\left(X_{\alpha_{1}}+X_{-\alpha_{1}}\right)+$ $\sin \pi / 2\left(X_{\alpha_{2}}+X_{-\alpha_{2}}\right)=X_{\alpha_{2}}+X_{-\alpha_{2}}$.

$$
\Phi_{m_{1}}^{+}=\Phi_{m_{2}}^{+} \circ \operatorname{Ad}(k)
$$

It is obvious that $\operatorname{Ad}(k)$ sends the roots of $m_{1}$ to the roots of $m_{2}$, so we need only to verify that it sends the positive roots into the positive roots. Let $\gamma=\gamma_{1} \circ \mathrm{Ad} u_{2}^{-1}$ $\in \Phi_{m_{2}}^{+}$. Since $k u_{1} k^{-1}=u_{2}$, then $\gamma_{1} \circ \operatorname{Ad} u_{2}^{-1} \operatorname{Ad}(k) \operatorname{Ad} u_{1}=\gamma_{1} \operatorname{Ad}(k)=s_{\beta} \gamma_{1}$. But $s_{\beta}=s_{\alpha_{1}} s_{\alpha_{2}} s_{\alpha_{1}}$ and $\gamma_{1} \neq \alpha_{1}, \alpha_{2}$ and is positive for $\Delta$, hence $s_{\beta} \gamma_{1} \in \Delta^{+}$. Similarly for the other inclusion. Note also that $\operatorname{Ad}(k) \mathfrak{n}_{1}=\mathfrak{n}_{2}$. Thus $\pi_{\xi_{2}, \nu_{2}} \simeq \pi_{\bar{\xi}_{2}, \bar{\nu}_{2}}$, where $\bar{\xi}_{2}$ is the $M_{1}$ representation of highest weight $\left.2 \delta_{n} \circ \operatorname{Ad}(k)\right|_{h_{1}^{-}}=s_{\beta} 2 \delta_{n_{n_{1}}}$ and $\bar{\nu}_{2}\left(X_{\alpha_{1}}+X_{-\alpha_{1}}\right)$ $=\nu_{2} \circ \operatorname{Ad}(k)\left(X_{\alpha_{1}}+X_{-\alpha_{1}}\right)=\nu_{2}\left(X_{\alpha_{2}}+X_{-\alpha_{2}}\right)$. 


$$
2 \delta_{\left.n\right|_{n_{1}^{-}}} \neq s_{\beta} 2 \delta_{\left.n\right|_{h_{-}^{-}}} .
$$

Indeed $2(\delta, \beta) /(\beta, \beta)=2$ and $2\left(\delta_{k}, \beta\right) /(\beta, \beta)=1$ ( $\beta$ is simple compact) forces $2\left(\delta_{n}, \beta\right) /(\beta, \beta)=1$. Thus $s_{\beta} 2 \delta_{n}=2 \delta_{n}-2 \beta$, and $2 \delta_{n_{\mid h_{\overline{1}}^{-}}}-s_{\beta} 2 \delta_{n_{h_{\overline{1}}}}=2 \beta_{\mid h_{\overline{1}}^{-}}=$ $2 \alpha_{2_{\mid h_{1}}} \neq 0$. But (2) says that $\pi_{\xi_{1}, \nu_{1}}$ is not equivalent to $\pi_{\bar{\xi}_{2}, \bar{\nu}_{2}}$ and hence to $\pi_{\xi_{2}, \nu_{2}}$, as asserted.

16.2. Let $\pi_{\xi_{1}, \nu_{1}}$ be as in the previous lemma and $\Psi_{1}^{+}=P \circ \operatorname{Ad} u_{1}^{-1}$. Then $\lambda_{1}=\alpha_{1} \circ \operatorname{Ad~} u_{1}^{-1}$ is the real positive root. Let $\Lambda\left(\xi_{1}, \nu_{1}\right)$ be the parameter for the infinitesimal character of $\pi_{\xi_{1}, \nu_{1}}$.

Corollary 16.3. $\Lambda\left(\xi_{1}, \nu_{1}\right)=\delta_{Q}$, where $Q$ is a positive root system for the roots of $\left(h_{1}^{-}+a_{1}\right)_{\mathbf{C}}$ and

(1) $Q \supset \Phi_{m_{1}}^{+}$and $\lambda_{1}$,

(2) $\lambda_{1}$ is $Q$ simple.

Proof. (1) is just Lemma 7.1.

$2\left(\nu_{1}, \lambda_{1}\right) /\left(\lambda_{1}, \lambda_{1}\right)=-\nu_{1}\left(X_{\alpha_{1}}+X_{-\alpha_{1}}\right)=1$ and hence (2) follows.

16.4. We now go back to our situation. Let $\Phi^{+}, \Phi_{m}^{+}$and $\alpha$ be as in $\S 10$.

Assuming that we are in the situation of Lemma 16.1, let $\pi_{\xi, \nu}$ be one of such principal series; then we have

LEMMA 16.5. $\Lambda(\xi, \nu)=\delta_{Q_{1}}$ where $Q_{1}$ is a positive root system for $\Phi$, containing $\Phi_{m}^{+}$ and $\alpha$ as a simple root.

Proof. To simplify the notation we may assume that $\pi_{\xi, \nu}=\pi_{\xi_{1}, \nu_{1}}$.

(1) $\exists k \in K$ such that $\operatorname{Ad}(k): h_{1}^{-}+a_{1} \rightarrow \mathfrak{h}^{-}+\mathfrak{a}$ and

(i) $\operatorname{Ad}(k)\left(X_{\alpha_{1}}+X_{-\alpha_{1}}\right)=2 h_{\alpha} /(\alpha, \alpha)\left(B\left(H, h_{\alpha}\right)=\alpha(H)\right)$.

(ii) $\Phi_{1}^{+}=\Psi_{1}^{+} \circ \operatorname{Ad}(k)^{-1}$ contains $\alpha$ and $\Phi_{m}^{+}$.

Assume for the moment that (1) is true, then $\alpha=\lambda_{1} \circ \operatorname{Ad}(k)^{-1}$ and $\pi_{\xi_{1}, \nu_{1}} \simeq \pi_{\bar{\xi}, \bar{\nu}}$ where $\bar{\xi}$ has highest weight $\Lambda_{\xi_{1}} \circ \operatorname{Ad}(k)^{-1}$ relative to $\Phi_{m}^{+}$and $\bar{\nu}=\nu_{1} \circ \operatorname{Ad}(k)^{-1}$. Since $\operatorname{Ad}(k) \mathfrak{n}_{1}=\mathfrak{n}$ (n defined in terms of $\alpha$ ) then $\Lambda(\bar{\xi}, \bar{\nu})=\lambda_{\xi_{1}} \circ \operatorname{Ad}(k)^{-1}+$ $\nu_{1} \operatorname{Ad}(k)^{-1}+\delta_{\Phi_{m}^{+}}=\Lambda\left(\xi_{1}, \nu_{1}\right) \circ \operatorname{Ad}(k)^{-1}=\delta_{Q} \circ \operatorname{Ad}(k)^{-1}$ where $Q$ is as in Corollary 16.3. Set $Q_{1}=Q \circ \operatorname{Ad}(k)^{-1}$, then $\delta_{Q} \circ \operatorname{Ad}(k)^{-1}=\delta_{Q_{1}}$ and the assertion follows by Corollary 16.3, modulo the proof of (1).

Let $k_{0} \in K$ such that $\operatorname{Ad}\left(k_{0}\right) \mathfrak{h}_{1}^{-}+a_{1}=\mathfrak{h}^{-}+\mathfrak{a}$.

(2) Clearly the roots of $\mathfrak{h}_{1}^{-}$are transformed under $\operatorname{Ad}\left(k_{0}\right)$ in roots of $\mathfrak{h}^{-}$. If $\Psi_{1}^{+} \circ \operatorname{Ad}\left(k_{0}\right)^{-1} \nsupseteq \Phi_{m}^{+}$, let $\sigma \in W\left(\Phi_{m}\right)$ so that $\sigma\left(\Psi_{1}^{+} \circ \operatorname{Ad}(k)^{-1} \cap \Phi_{m}\right)=\Phi_{m}^{+}$. Let $m \in N_{M}(T)$ so that $\operatorname{Ad}(m)_{\mid \mathfrak{h}^{-}}=\sigma(T$ the analytic subgroup of $M$ corresponding to $\mathfrak{h}^{-}$and $N_{M}(T)$ its normalizer in $\left.M\right)$. Then $\operatorname{Ad}\left(m k_{0}\right)\left(\mathfrak{h}_{1}^{-}+\mathfrak{a}_{1}\right)=\mathfrak{h}^{-}+\mathfrak{a}$ and $\sigma \Psi_{1}^{+} \circ \operatorname{Ad}\left(k_{0}\right)^{-1} \cap \Phi_{m}=\sigma\left(\Psi_{1}^{+} \circ \operatorname{Ad}\left(k_{0}\right)^{-1} \cap \Phi_{m}\right)=\Phi_{m}^{+}$since $\sigma$ preserves the roots of $m$. (2) forces $\lambda_{1} \circ \operatorname{Ad}\left(m k_{0}\right)^{-1}=\lambda_{1} \circ \operatorname{Ad}(k)^{-1}= \pm \alpha$.

If $\lambda_{1} \circ \operatorname{Ad}\left(m k_{0}\right)^{-1}=\alpha$ then (1) is true for $k=m k_{0}$. If $\lambda_{1} \circ \operatorname{Ad}\left(m k_{0}\right)^{-1}=-\alpha$, let $s_{\alpha}$ be the reflection by $\alpha$ and $k_{1} \in K$ so that $\operatorname{Ad}\left(k_{1}\right)_{\mid b^{-}+a}=s_{\alpha_{\mid b}-+a}$ $\left(k_{1}=\exp \left[\pi / \sqrt{2}(\alpha, \alpha)^{1 / 2}\right]\left(X_{\alpha}+\theta X_{\alpha}\right)\right)$. Then $\operatorname{Ad}\left(k_{1} m k_{0}\right)\left(\mathfrak{h}_{1}^{-}+a_{1}\right)=\mathfrak{h}^{-}+a$ and (i) is true. Since $s_{\lambda_{0}}$ acts trivially on $\Phi_{m}$, we still have $\Psi_{1}^{+} \circ \operatorname{Ad}\left(k_{1} m k_{0}\right)^{-1} \cap \Phi_{m}=$ $\Phi_{m}^{+}$; thus (1) is true for $k=k_{1} m k_{0}$ and the proof is complete. 
17. Some more results.

17.1. If $\mu \in \mathrm{a}_{\mathbf{C}}^{*}$ then $\mu=\left.\mu_{1} \alpha\right|_{\mathfrak{a}}$, where $\mu_{1} \in \mathbf{C}$. We abuse notation and set $\mu=2 \mu_{1}$.

Let $\pi_{i, j}=\pi_{\Lambda_{i, I_{\mid}-}, \nu_{i, j}}$ be the principal series associated to $P^{i, j}$, defined for each group as in $\$ 13$.

We also denote by $H^{i, j}$ the representation space of $\pi_{i, j}$.

Let $\pi_{i}$, for $i$ in the right range, the discrete series representation parametrized by $\delta_{._{i}}$ (. stands for one of the positive root systems introduced for each group in $\$ 11$ ). Denote by $H^{i}$ the representation space. There is an easy way of reading off the formula of Theorem 9.1, using the diagrams of $\S 13$. We associate a \pm sign to each $P^{i, j}$ in the following way: assign a + sign to $P^{0,1}$ and suppose we have given a sign to $P^{i, j}$, then associate to $P^{i j+1}$ and to $P^{i+1, j}$ (when it is defined) - the sign of $P^{i, j}$. Set $\tau=\delta_{P^{0,1}} \circ$ Ad $u_{\lambda}(\lambda$ as in $\S 10)$; then the formula of Theorem 9.1 reads:

$$
\sum \varepsilon_{i, j} \theta_{i, j}+(-1)^{q} \sum \theta_{i}=1,
$$

where $\theta_{i, j}\left(\theta_{i}\right)$ is the character of $\pi_{i, j}\left(\pi_{i}\right), \varepsilon_{i, j}$ is the sign associated to $P^{i j}$ and the sums are taken over $i, j$ in the right range. The result is immediate since the diagrams are built in such a way that two positive chambers are connected by a line precisely when one is obtained from the other by reflection of a simple root.

Let $\xi \in \hat{M}, \nu \in a_{C}^{*}$ such that $\operatorname{Re} \nu>0$. If $f \in H_{F}^{\xi, \nu}$ define $(A(\nu) f(g))=$ $\int_{\bar{N}} f(g \bar{n}) d \bar{n}$, where $g \in G$ and $\bar{N}$ is the analytic subgroup of $G$ corresponding to $\bar{n}=\theta(\mathfrak{n})$. Denote by $J^{\xi, \nu}$ the only irreducible quotient of $\left(\pi_{\xi, \nu}, H_{F}^{\xi, \nu}\right)$ as by Theorem 6.5. Then if $Z_{\xi, \nu}=\operatorname{ker} A(\nu)$ we have $J^{\xi, \nu}=H_{F / Z_{\xi, \nu}}^{\xi, \nu} J^{\xi, \nu}$ is the Langlands quotient.

Due to Langlands' classification of irreducible quasisimple representation [12] and [11] the following result is evident:

THEOREM 17.3. If $\pi_{\xi, \nu}$ has a regular infinitesimal character, then the only irreducible components of $\pi_{\xi, \nu}$ are either Langlands quotients or discrete series representations.

We also have

THEOREM 17.4. Let $\nu, \nu^{\prime} \in a^{*}$ such that $\nu, \nu^{\prime}>0$. Let $\pi$ be a representation of $G$ infinitesimally equivalent to $J^{\xi, \nu}$. Then if $\pi$ is a subquotient of $Z_{\xi^{\prime}, \nu^{\prime}}$ we have $\nu^{\prime}>\nu$ $\left(\xi^{\prime} \in \hat{M}\right)$.

Proof. The theorem is an immediate consequence of the well-known result on matrix coefficients of a principal series: let $\lambda \in \Lambda^{+}$be a simple root and $H_{\lambda} \in \mathfrak{a}$ such that $\lambda\left(H_{\lambda}\right)=1$, then $\lim _{t \rightarrow \infty} e^{t(\rho-z)}\left\langle\pi_{\xi, z}\left(a_{t}\right) f, g\right\rangle=\langle A(z) f(1), g(1)\rangle$ where $z$ $\in a_{C}^{*}, \operatorname{Re} z>0, f, g H_{F}^{\xi, z}$ and $a_{t}=\exp t H_{\lambda}$.

Write $J^{i, j}$ for the Langlands quotient of $\pi_{i, j}$.

COROLlaRY 17.5. (1) If $i \neq 0, j \neq 1$ then there are an even number of subquotients in $\left\{\pi_{k, t}\right\}$ infinitesimally equivalent to $J^{i j}$.

(2) $J^{0,1}$ is an irreducible component only of $\pi_{0,1}$.

Proof. (2) is nothing else than Theorem 17.4.

Since $J^{0,1}$ is the trivial representation [9], this is consistent with 17.2. 
To prove (1) use 17.2 to get $\sum \varepsilon_{i j} m_{i j}$ (character of $\left.J^{i, j}\right)=0\left(J^{i j}\right.$ are infinitesimally inequivalent).

We are now ready to prove some results for $F_{4}$ and $\operatorname{Sp}(n, 1)$.

LEMMA 17.6. $G=F_{4}$. Let $\pi_{i}, 1 \leqslant i \leqslant 3$, be the discrete series representation associated to $\delta_{F_{i}}(c f$. Lemma 11.11). Then

(1) $\pi_{3}$ is a subspace of $\pi_{0,1}$,

(2) $\pi_{2}$ is not an irreducible component of $\pi_{0,1}$.

Proof. Let $\lambda_{1}, \lambda_{2}, \lambda_{3}, \lambda_{4}$ be the fundamental weights for $\Delta_{k}^{+}$, in particular $\lambda_{1}=\varepsilon_{1}$ and $\lambda_{4}=\frac{1}{2}\left(\varepsilon_{1}+\varepsilon_{2}+\varepsilon_{3}+\varepsilon_{4}\right)$. If $m, k \in \mathbf{Z}_{+}$and $m-k \in 2 \mathbf{Z}$, denote by $X_{m, k}$ the irreducible $K$-module of highest weight $(m-k) \lambda_{1} / 2+k \lambda_{4}=m \varepsilon_{1} / 2+$ $k\left(\varepsilon_{2}+\varepsilon_{3}+\varepsilon_{4}\right) / 2$.

Let

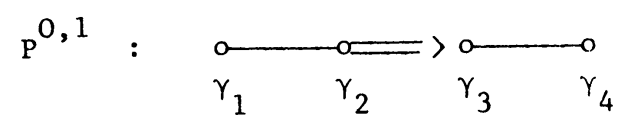

as in 13.9, thus $\lambda=\gamma_{\left.4\right|_{a}}$ is the simple root for $a$ and $\alpha=2 \lambda$. Since $\nu_{0,1}=11 \lambda$ and $2 \rho=22 \lambda$, thus in [9] it is shown that $\pi_{0,1}$ has a composition series by $H^{0,1} \supset \tilde{W}_{0} \supset$ $\tilde{M}_{0} \supset(0)$ where

$$
\tilde{M}_{0}=\sum_{m-k>6} X_{m, k}, \quad \tilde{W}_{0}=\sum_{m+k>0} X_{m, k}, \quad \text { and } \quad \tilde{W}_{0} / \tilde{M}_{0}=\sum_{\substack{m-k<6 \\ m+k>0}} X_{m, k} .
$$

(a) $\tilde{M}_{0} \simeq^{g} \pi_{3}$. Since the minimal $K$-type $\tau_{2 \delta_{n}^{F_{3}}}$ of $\pi_{3}$ has highest weight $2 \delta_{n}^{F_{3}}=4 \varepsilon_{1}$, it is immediate that $X_{8,0} \simeq \tau_{2 \delta_{n} F_{3}}$ with $X_{8,0} \subset \tilde{M}_{0}$. If we can prove that $\tilde{M}_{0}$ does not contain any irreducible $K$-module of highest weight of the form $2 \delta_{n}^{F_{3}}-A$, where $A$ stands for a nonempty sum of positive roots for $F_{3}$, then (a) will follow from [15, Theorem 13].

A generic element of the form $A$ is

$$
\sum_{i=1}^{4} k_{i} \gamma_{i}=\frac{1}{2} k_{4} \varepsilon_{1}+\left(k_{1}-\frac{k_{4}}{2}\right) \varepsilon_{2}+\left(k_{2}-k_{1}-\frac{k_{4}}{2}\right) \varepsilon_{3}+\left(k_{3}-k_{2}-\frac{k_{4}}{2}\right) \varepsilon_{4},
$$

where $k_{i} \in \mathbf{Z}_{+}$, and they are not all zero.

$$
\begin{aligned}
2 \delta_{n}^{F_{3}}-A= & \left(4-\frac{1}{2} k_{4}\right) \varepsilon_{1}+\left(\frac{1}{2} k_{4}-k_{1}\right) \varepsilon_{2} \\
& +\left(\frac{1}{2} k_{4}+k_{1}-k_{2}\right) \varepsilon_{3}+\left(\frac{1}{2} k_{4}+k_{2}-k_{3}\right) \varepsilon_{4} .
\end{aligned}
$$

Assume that there exist $m, k \in \mathbf{Z}_{+}$such that $X_{m, k} \subset \tilde{M}_{0}$ and $X_{m, k}$ is equivalent to the $K$-type of highest weight $2 \delta_{n}^{F_{3}}-A$. Then we would have:

$$
m=8-k_{4}, \quad k=k_{4}-2 k_{1}, \quad k_{1}+k_{3}=2 k_{2}, \quad k_{3}=3 k_{1} .
$$

But $m-k>6$ forces $k_{4}-k_{1}<1$. Since $k \in Z_{+}$we also have $k_{4}>2 k_{1}$. Thus $2 k_{1} \leqslant k_{4}<k_{1}+1$.

This last inequality is satisfied only if $k_{1}=0$ and hence $k_{2}=k_{3}=k_{4}=0$. Therefore $\tilde{M}_{0}$ does not contain any irreducible $K$-module of highest weight of the form $2 \delta_{n}^{F_{3}}-A$ and (a) follows. 
(b) $\tilde{W}_{0} / \tilde{M}_{0}$ is not infinitesimally equivalent to a discrete series.

If $\tilde{W}_{0} / \tilde{M}_{0}$ is a discrete series, it could only be $\pi_{2}$ [cf. Lemma 15.9]. Now $2 \delta_{n}^{F_{2}}=3 \varepsilon_{1}+\varepsilon_{2}+\varepsilon_{3}+\varepsilon_{4}$. Hence:

(i) $X_{6,2} \simeq \tau_{2 \delta_{n}^{F}}$,

(ii) $X_{7,1}$ is equivalent to the $K$-module of highest weight $2 \delta_{n}^{F_{2}}+$ $\frac{1}{2}\left(\varepsilon_{1}-\varepsilon_{2}-\varepsilon_{3}-\varepsilon_{4}\right)$. Since $-\frac{1}{2}\left(\varepsilon_{1}-\varepsilon_{2}-\varepsilon_{3}-\varepsilon_{4}\right)$ is positive for $F_{2}$, the result follows again by [15, Theorem 1.3].

Proposition 17.7. $G=F_{4}$. Let $\tau_{\mu}$ be the irreducible $K$-module of highest weight $\mu=2 \varepsilon_{1}+2 \varepsilon_{2}+2 \varepsilon_{3}+2 \varepsilon_{4}$, then $\tau_{\mu}$ is a K-type of $\pi_{2}$.

Proof. To prove that $\tau_{\mu}$ is a $K$-type of $\pi_{2}$, we use Blattner's multiplicity formula [6]. Recall that

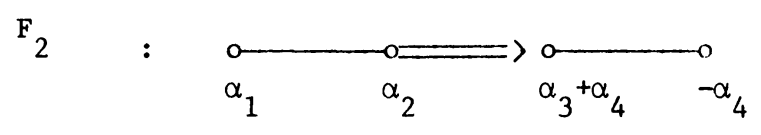

and

$$
2 \delta_{n}^{F_{2}}=3 \varepsilon_{1}+\varepsilon_{2}+\varepsilon_{3}+\varepsilon_{4}, \quad \delta_{k}=\frac{1}{2}\left(7 \varepsilon_{1}+5 \varepsilon_{2}+3 \varepsilon_{3}+\varepsilon_{4}\right)
$$

If $P$ is the usual partition function relative to the positive noncompact roots for $F_{2}$, then Blattner's multiplicity formula says

$$
m_{2 \delta_{n} F_{2}}(\mu)=\sum_{w \in W_{k}} P\left(w\left(\mu+\delta_{k}\right)-2 \delta_{n}^{F_{2}}-\delta_{k}\right)
$$

Write $P(w)=P\left(w\left(\mu+\delta_{k}\right)-2 \delta_{n}^{F_{2}}-\delta_{k}\right)$. Now if $w \in W_{k}$, thus

$$
w\left(\sum_{i=1}^{4} a_{i} \varepsilon_{i}\right)=\sum_{i=1}^{4}(-1)^{v_{i}^{w}} a_{i} \varepsilon_{\sigma^{w}(i)} \quad \text { with } v_{i}^{w}=0,1(i=1, \ldots, 4),
$$

and $\sigma^{w}$ a permutation of $\{1, \ldots, n\}$. We write $w=v^{w}, \sigma^{w}$. A sum of positive noncompact roots is of the form: $\sum_{i=1}^{4} \frac{1}{2}\left(x_{i} \varepsilon_{i}\right)$ where

$$
\begin{aligned}
& x_{1}=k_{1}+k_{2}+k_{3}+k_{4}+k_{5}+k_{6}+k_{8}-k_{7}, \\
& x_{2}=k_{1}+k_{4}+k_{5}+k_{7}+k_{8}-k_{2}-k_{3}-k_{6}, \\
& x_{3}=k_{1}+k_{2}+k_{5}+k_{6}+k_{7}-k_{3}-k_{4}-k_{8}, \\
& x_{4}=k_{1}+k_{2}+k_{3}+k_{4}+k_{7}-k_{5}-k_{6}-k_{8},
\end{aligned}
$$

with $k_{i} \in \mathbf{Z}_{+}, i=1, \ldots, 8$.

$$
2 \delta_{n}^{F_{2}}+\delta_{k}=\frac{1}{2}\left\{13 \varepsilon_{1}+7 \varepsilon_{2}+5 \varepsilon_{3}+3 \varepsilon_{4}\right\}, \quad \mu+\delta_{k}=\frac{1}{2}\left\{11 \varepsilon_{1}+9 \varepsilon_{2}+7 \varepsilon_{3}+5 \varepsilon_{4}\right\} \text {. }
$$

Thus

$$
\begin{aligned}
& w\left(\mu+\delta_{k}\right)-2 \delta_{n}^{F_{2}}-\delta_{k}=(-1)^{v_{1}^{w} \frac{11}{2} \varepsilon_{\sigma^{w}(1)}}+(-1)^{v_{2}^{w} \frac{9}{2} \varepsilon_{\sigma^{w}(2)}}+(-1)^{v_{3}^{w} \frac{7}{2} \varepsilon_{\sigma^{w}(3)}} \\
& +(-1)^{0 * \frac{5}{2}} \varepsilon_{\sigma^{w(4)}}-\frac{13}{2} \varepsilon_{1}-\frac{7}{2} \varepsilon_{2}-\frac{5}{2} \varepsilon_{3}-\frac{3}{2} \varepsilon_{4} \text {. }
\end{aligned}
$$


Suppose that $\sigma^{w}=I$, thus $P(w) \neq 0$ iff there exists $k_{j} \in \mathbf{Z}_{+}$such that

$$
\begin{aligned}
& (-1)^{v_{1}^{\mathrm{w}}} 11-13=x_{1}, \quad(-1)^{v_{1}^{\mathrm{N}}} 11-13=x_{1} \text {, } \\
& (-1)^{v_{2}^{\mu}} 9-7=x_{2} \text {, i.e. } \quad(-1)^{v_{1}^{\omega}} 11+(-1)^{v_{2}^{\omega}} 9-20=x_{1}+x_{2} \text {, } \\
& (-1)^{v_{3}^{*}} 7-5=x_{3} \text {, i.e. } \quad(-1)^{v_{1}^{w}} 11+(-1)^{v_{3}^{*}} 7-18=x_{1}+x_{3} \text {, } \\
& (-1)^{v_{4}^{*}} 5-3=x_{4}, \quad(-1)^{v_{1}^{w}} 11+(-1)^{v_{4}^{w}} 5-16=x_{1}+x_{4} \text {. }
\end{aligned}
$$

Since $x_{1}+x_{i} \geqslant 0, i=2,3,4$, the last three equations force $v_{i}^{w}=0, i=1,2,3,4$, and $k_{1}=k_{2}=k_{3}=k_{4}=k_{5}=k_{6}=k_{8}=0$. Substituting these values in the first equation we get $k_{7}=2$. Thus we have proved that

$$
\sum_{\substack{w \in W_{k} \\ w=v^{w}}} P(w)=1 .
$$

Suppose that $\sigma^{w} \neq I$, then we will prove that $\sigma(4)=4$.

(a) If $\sigma^{w}(4)=1$ then $(*)(-1)^{v *} 5-13=x_{1}$.

On the other hand we would have $\sigma^{w}(1)=4$ or $\sigma^{w}(2)=4$ or $\sigma^{w}(3)=4$. If $\sigma^{w}(1)=4$ then $\sigma^{w}(3)=2$ or 3 . If $\sigma^{w}(3)=2$ then $(-1)^{v_{3}^{w}} 7-7=x_{2}$ and this equation summed with (*) gives a contradiction, since $x_{1}+x_{2} \geqslant 0$. If $\sigma^{w}(3)=3$ then $(-1)^{v_{3}^{w}} 7-5=x_{3}$ and this again is impossible $\left(x_{1}+x_{3} \geqslant 0\right)$. Thus $\sigma^{w}(1) \neq 4$.

If $\sigma^{w}(2)=4\left(\sigma^{w}(3)=4\right)$ again we get a contradiction $\left(x_{1}+x_{4}>0\right)$ summing the equation $\sigma^{w}(2)=4\left(\sigma^{w}(3)=4\right)$ with $(*)$. Therefore $\sigma^{w}(4) \neq 1$.

(b) If $\sigma^{w}(4)=2$ then $\sigma^{w}(1)=1$ or $\sigma^{w}(2)=1$ or $\sigma^{w}(3)=1$. In all cases we get a contradiction $\left(x_{1}+x_{2} \geqslant 0\right)$ summing the equation in question with $\sigma^{w}(4)=2$. Thus $\sigma^{w}(4) \neq 2$.

The same kind of argument proves that $\sigma^{w}(4) \neq 3$. If $\sigma^{w}(4)=4$ then $\sigma^{w}(2) \neq 1$ and $\sigma^{w}(3) \neq 1$, as is easily seen. Thus we have proved that if $\sigma^{w} \neq I$ then $\sigma^{w}(4)=4, \sigma^{w}(1)=1$ and $\sigma^{w}(2)=3, \sigma^{w}(3)=2$. In particular

$$
(-1)^{v_{1}^{w}} 11-13=x_{1}, \quad(-1)^{v_{3}^{*}} 7-7=x_{2} \text {, }
$$

which is impossible. Hence

$$
\sum_{\substack{w \in W_{k} \\ w=w^{w} \sigma^{w} \\ \sigma^{w} \neq I}} P(w)=0
$$

and we can conclude that $m_{2 \delta_{n}{ }_{2}}(\mu)=1$.

Corollary 17.8. $\pi_{2}$ is an irreducible component of $\pi_{2,6}, \pi_{1,6}$ and $\pi_{1,7}$ and it is not an irreducible component of any other principal series.

Proof. Combining Theorem 12.5 and Lemmas 15.9 and 17.6 we have that $\pi_{2}$ could be an irrreducible component only of the following principal series:

$$
\pi_{0,2}, \pi_{0,3}, \pi_{0,4}, \pi_{0,5}, \pi_{1,4}, \pi_{1,5}, \pi_{1,6}, \pi_{1,7} \text { and } \pi_{2,6} \text {. }
$$

Let us consider the $K$-type $\tau_{\mu}$ of the previous proposition and compute its $M$-types. Let $a_{1}=a_{2}=a_{3}=a_{4}=4$.

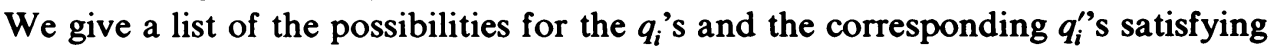
(i)-(iv) as in the proof of Theorem 12.5. 


$\begin{array}{cccccccc}q_{1} & q_{2} & q_{3} & q_{4} & q_{1}^{\prime} & q_{2}^{\prime} & q_{3}^{\prime} & q_{4}^{\prime} \\ 2 & 2 & 2 & 2 & 2 & 2 & 2 & 2 \\ 2 & 2 & 2 & 1 & \frac{5}{2} & \frac{3}{2} & \frac{3}{2} & \frac{3}{2} \\ 2 & 2 & 2 & 0 & 3 & 1 & 1 & 1 \\ 2 & 2 & 2 & -1 & \frac{7}{2} & \frac{1}{2} & \frac{1}{2} & \frac{1}{2} \\ 2 & 2 & 2 & -2 & 4 & 0 & 0 & 0\end{array}$

Let $b_{1}, b_{2}, b_{3}$ satisfy (i)-(iv) as in Theorem 12.5 , then $\left(b_{1}, b_{2}, b_{3}\right)$ is one of the following triple:

$$
\begin{aligned}
& (2,2,2), \quad\left(\frac{5}{2}, \frac{3}{2}, \frac{3}{2}\right), \quad\left(\frac{3}{2}, \frac{3}{2}, \frac{3}{2}\right), \quad(3,1,1),(2,1,1), \\
& (1,1,1), \quad\left(\frac{7}{2}, \frac{1}{2}, \frac{1}{2}\right), \quad\left(\frac{5}{2}, \frac{1}{2}, \frac{1}{2}\right), \quad\left(\frac{3}{2}, \frac{1}{2}, \frac{1}{2}\right), \quad\left(\frac{1}{2}, \frac{1}{2}, \frac{1}{2}\right), \\
& (4,0,0), \quad(3,0,0), \quad(2,0,0), \quad(1,0,0), \quad(0,0,0) .
\end{aligned}
$$

Thus by Theorem 10.9, the $M$-types of $\tau_{\mu}$ are of the form $\tau_{\gamma} \circ \phi$, where $\gamma=$ $\sum_{j=1}^{3} b_{i} \varepsilon_{i}$, with $\left(b_{1}, b_{2}, b_{3}\right)$ in the above list. They all have multiplicity one. $\tau_{\mu}$ is a $K$-type, with multiplicity one, of the following principal series.

$$
\pi_{0,1} \pi_{0,2} \pi_{1,4} \pi_{1,6} \pi_{1,7} \pi_{2,4} \pi_{2,6}
$$

(use the Frobenius reciprocity theorem and the list of the $\Lambda_{i j}$ 's given in the proof of Lemma 15.9).

(a) $\tau_{\mu}$ is a $K$-type of $J^{0,2}$ and $J^{0,2}$ is an irreducible component of $\pi_{0,1} \cdot J^{0,2}$ is by definition in $\pi_{0,2}$ and Theorem 17.4 says that the only other place where $J^{0,2}$ may appear is in $\pi_{0,1}$. But $J^{0,2}$ appears in $\pi_{0,2}$ only once by [16] (it can also be proved directly), then Corollary 17.5 says that $J^{0,2}$ is in $\pi_{0,1}$. Referring to the proof of Lemma 17.3 , since $H^{0,1} / \tilde{W}_{0}=J^{0,1}$, and $\tilde{M}_{0} \simeq \pi_{3}$, then

$$
J^{0,2} \simeq \tilde{W}_{0} / \tilde{M}_{0}=\sum_{\substack{m-k<6 \\ m+k>0}} X_{m, k}
$$

Now $\tau_{\mu}=X_{4,4} \subset \tilde{W}_{0} / \tilde{M}_{0}$, so (a) is true.

(b) $\tau_{\mu}$ is a $K$-type of $J^{2,4}$ and $J^{2,4}$ is an irreducible component of $\pi_{1,4}$. We know by Lemma 15.9 that there are no discrete series in the composition series of $\pi_{2,4}$.

Since $\tau_{\mu}$ is a $K$-type of $\pi_{2,4}$ then $\tau_{\mu}$ is a $K$-type of an irreducible component of $\pi_{2,4}$, which is necessarily a Langlands quotient. On the other hand $\tau_{\mu}$ is a $K$-type of $\pi_{2}$ and $\pi_{2}$ appears in $\pi_{2,6}$ and $\pi_{1,7}\left(\alpha=\tilde{\varepsilon}_{1}\right.$ is simple for $P^{1,7}$ and $P^{2,5}$ so apply 16.6), thus $\tau_{\mu}$ is not a $K$-type of $J^{2,6}$ and $J^{1,7}$ (the multiplicity of $\tau_{\mu}$ is always one). Thus if $\tau_{\mu}$ is not a $K$-type of $J^{2,4}$ we are forced to conclude that the irreducible component of $\pi_{2,4}$ of which $\tau_{\mu}$ is a $K$-type is $J^{1,6}$. But it is easily seen to be false using the $K$-type of highest weight $\gamma=3 \varepsilon_{1}+\varepsilon_{2}+\varepsilon_{3}$ which is a $K$-type of $J^{1,6}$ and not of $\pi_{2,4}$. Therefore $\tau_{\mu}$ is a $K$-type of $J^{2,4}$ and (b) follows from (a) together with Corollary 17.5 .

Using (1) and (2) we know that $\pi_{2}$ can appear only in $\pi_{0,2}, \pi_{1,4}, \pi_{1,6}, \pi_{1,7}, \pi_{2,6}$. But (a) and (b) say that $\pi_{2}$ cannot be in $\pi_{0,2}$ and $\pi_{1,4}$. The result is now a consequence of Lemma 16.6 and Corollary 9.3. 
THEOREM 17.9. $A$ discrete series representation is realized in a nonunitary principal series with multiplicity at most one.

Proof. In view of the result of $\S 8$, we only need to prove the theorem for discrete series representation with trivial infinitesimal character. With this in mind for $\operatorname{Spin}(2 n, 1), \operatorname{SU}(n, 1)$ and $\operatorname{Sp}(n, 1)$ the result is a consequence of the fact that the $M$-types of the minimal $K$-type all have multiplicity one. For $F_{4}$, the only discrete series which could occur twice is $\pi_{2}$ in $\pi_{0,2}$ as we noted at the end of the proof of Lemma 15.9. But now Corollary 17.8 applies to give the result.

Proposition 17.10. $G=\operatorname{Sp}(n, 1)$. The discrete series $\pi_{n-1}$ is an irreducible component of $\pi_{n-1, n+1}, \pi_{n-2, n+2}, \pi_{n-2, n+1}$ and it is not an irreducible component of any other principal series.

Proof. We prove that there exists a $K$-type of $\pi_{n-1}$ which is not a $K$-type of $H^{n-2, n-1}, H^{n-2, n}, H^{n-3, n-1}, H^{n-3, n}$ (these principal series are the only ones where $\pi_{n-1}$ could occur, besides the ones in the statement).

Let $\mu=\sum_{i=1}^{n} 2 \varepsilon_{i}+4 \varepsilon_{n+1}$, and consider the irreducible $K$-module $\tau_{\mu}$ with highest weight $\mu$. The choice of such $\mu$ is the obvious one, considering the form of the $M$-types that determine the above principal series. To prove that $\tau_{\mu}$ is a $K$-type of $\pi_{n-1}$, we use Blattner's multiplicity formula (cf. [6]). Recall that

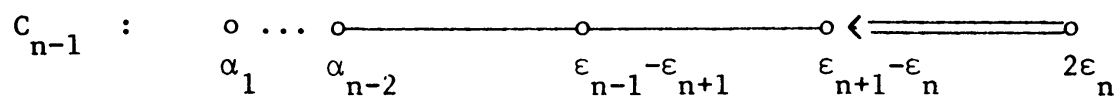

and

$$
2 \delta_{n}^{C_{n-1}}=\sum_{j=1}^{n-1} 2 \varepsilon_{j}+2 \varepsilon_{n+1}, \quad \delta_{k}=\sum_{j=1}^{n}(n-j+1) \varepsilon_{j}+\varepsilon_{n+1} .
$$

If $P$ is the usual partition function relative to the positive noncompact roots for $C_{n-1}$, then Blattner's multiplicity formula says

$$
m_{2 \delta_{n}^{c_{n-1}}}(\mu)=\sum_{w \in W_{k}} P\left(w\left(\mu+\delta_{k}\right)-2 \delta_{n}^{C_{n-1}}-\delta_{k}\right) \quad\left(W_{k}=W\left(\Delta_{k}\right)\right) .
$$

Write $P(w)=P\left(w\left(\mu+\delta_{k}\right)-2 \delta_{n}^{C_{n-1}}-\delta_{k}\right)$. Now

$$
\Delta_{k}^{+}: \underset{\alpha_{1}}{\circ} \cdots \underset{\alpha_{n-1}}{\circ} \underset{2 e_{n}}{\stackrel{\circ}{\circ}} \stackrel{\circ}{\alpha_{n+1}}
$$

and if $w \in W_{k}$, thus:

$$
w\left(\sum_{i=1}^{n+1} a_{i} \varepsilon_{i}\right)=\sum_{i=1}^{n}(-1)_{a_{i}^{*}}^{v_{i}^{w}} \varepsilon_{\sigma^{w}(i)}+(-1)_{a_{n+1}^{0}}^{v_{n+1}^{n}} \varepsilon_{n+1},
$$

with $v_{i}^{w}=0,1(1 \leqslant i \leqslant n+1)$ and $\sigma^{w}$ a permutation of the set $\{1, \ldots, n\}$. We write $w=v^{w} \cdot \sigma^{w}$. The positive noncompact roots for $C_{n-1}$ are

$$
\varepsilon_{1} \pm \varepsilon_{n+1}, \varepsilon_{2} \pm \varepsilon_{n+1}, \ldots, \varepsilon_{n-1} \pm \varepsilon_{n+1}, \varepsilon_{n+1} \pm \varepsilon_{n} \text {. }
$$


Thus a sum of positive noncompact roots will be of the form

$$
\begin{aligned}
& \sum_{j=1}^{n-1} k_{j}\left(\varepsilon_{j}-\varepsilon_{n+1}\right)+k_{n}\left(\varepsilon_{n+1}-\varepsilon_{n}\right)+\sum_{j=n+1}^{2 n-1} k_{j}\left(\varepsilon_{j}+\varepsilon_{n+1}\right)+k_{2 n}\left(\varepsilon_{n+1}+\varepsilon_{n}\right) \\
& =\sum_{j=1}^{n-1}\left(k_{j}+k_{n+j}\right) \varepsilon_{j}+\left(k_{2 n}-k_{n}\right) \varepsilon_{n}+\left(k_{n}+\cdots+k_{2 n}-k_{1}-\cdots-k_{n-1}\right) \varepsilon_{n+1},
\end{aligned}
$$

with $k_{j} \in \mathbf{Z}_{+}$for $1 \leqslant j \leqslant 2 n$.

$$
\begin{aligned}
2 \delta_{n}^{C_{n-1}}+\delta_{k} & =\sum_{j=1}^{n-1}(n-j+3) \varepsilon_{j}+\varepsilon_{n}+3 \varepsilon_{n+1}, \\
\mu+\delta_{k} & =\sum_{j=1}^{n}(n-j+3) \varepsilon_{j}+5 \varepsilon_{n+1} .
\end{aligned}
$$

Thus

$$
\begin{aligned}
w\left(\mu+\delta_{k}\right)-2 \delta_{n}^{C_{n-1}}-\delta_{k}= & \sum_{j=1}^{n}(-1)^{v_{j}^{\omega}}(n-j+3) \varepsilon_{\sigma}{ }^{\omega}(j) \\
& +(-1)^{v_{n+1}^{w}} \cdot 5 \varepsilon_{n+1}-\sum_{j=1}^{n-1}(n-j+3) \varepsilon_{j}-\varepsilon_{n}-3 \varepsilon_{n+1} .
\end{aligned}
$$

Suppose that $\sigma^{w}=I$, thus $P(w) \neq 0$ iff there exists $k_{j} \in \mathbf{Z}_{+}$such that

$$
\begin{gathered}
(-1)^{v_{j}^{\omega}(n-j+3)}-(n-j+3)=k_{j}+k_{n+j} \quad(1<j \leqslant n-1), \\
(-1)^{v_{n}^{w}} \cdot 3-1=k_{2 n}-k_{n}, \\
(-1)^{v_{n+1}^{\omega}} \cdot 5-3=k_{n}+\cdots+k_{2 n}-k_{1}-\cdots-k_{n-1} .
\end{gathered}
$$

Since $k_{j} \geqslant 0$, the first $n-1$ equations force $v_{j}^{w}=0$ and $k_{j}=k_{j+n}=0$ for $j=$ $1, \ldots, n-1$. Substituting these values in the last two equations we have

$$
(-1)^{v_{n}^{w}} \cdot 3-1=k_{2 n}-k_{n}, \quad(-1)^{v_{n+1}^{w}} \cdot 5-3=k_{n}+k_{2 n} \text {. }
$$

Again, since $k_{n}, k_{2 n} \geqslant 0$ we have $v_{n+1}^{w}=0$ and hence

$$
(-1)^{v_{n}^{w}} \cdot 3-1=k_{2 n}-k_{n}, \quad 2=k_{n}+k_{2 n},
$$

i.e.

$$
(-1)^{v_{n}^{w}} 3-1=2-2 k_{n}, \quad 2=k_{n}+k_{2 n} \text {. }
$$

If $v_{n}^{w}=0$, thus $k_{n}=0$ and $k_{2 n}=2$. If $v_{n}^{w}=1$, thus $k_{n}=3$ and $k_{2 n}=-1$ which contradicts the positivity of $k_{2 n}$. Thus we have proved that

$$
\sum_{\substack{w \in W_{k} \\ w=v^{w I}}} P(w)=1 .
$$

Suppose now that $\sigma^{w} \neq I$. Thus there exist $j \in\{1, \ldots, n\}$ so that $\sigma(j)=l$ for some $l<j$. If the partition function for such $w$ were different from zero, thus the following equation would be satisfied for some $k_{l}, k_{l+n} \in \mathbf{Z}_{+}$:

$$
(-1)^{v_{j},}(n-j+3)-(n-l+3)=k_{l}+k_{l+n} \text {, }
$$


and this is clearly impossible. Thus

$$
\sum_{\substack{w \in W_{k} \\ w=w^{w} \sigma^{w} \\ \sigma^{w} \neq I}} P(w)=0
$$

and we can conclude that $m_{2 \delta_{n-1}} c_{n-1}(\mu)=1$.

To find the $M$-types of $\tau_{\mu}$, we use the computations of Lemma 12.4(4) to see that $b_{1}, b_{2}, \ldots, b_{n}$ could be

(i) $(0,2, \ldots, 2,0)$,

(ii) $(1,2, \ldots, 2,1)$,

(iii) $(2,2, \ldots, 2,2)$.

Since $a_{n+1}=4$, by (i) get $b_{0}=2$, by (ii) get $b_{0}=\frac{5}{2}, \frac{3}{2}$, and by (iii) get $b_{0}=3,2,1$. Hence the $M$-types of $\tau_{\mu}$ are:

$$
\begin{gathered}
3\left(\varepsilon_{1}+\varepsilon_{n+1}\right)+\sum_{j=2}^{n-1} 2 \varepsilon_{j}+2 \varepsilon_{n}, \\
2\left(\varepsilon_{1}+\varepsilon_{n+1}\right)+\sum_{j=2}^{n-1} 2 \varepsilon_{j}+2 \varepsilon_{n}, \\
\left(\varepsilon_{1}+\varepsilon_{n+1}\right)+\sum_{j=2}^{n-1} 2 \varepsilon_{j}+2 \varepsilon_{n}, \\
\frac{5}{2}\left(\varepsilon_{1}+\varepsilon_{n+1}\right)+\sum_{j=2}^{n-1} 2 \varepsilon_{j}+\varepsilon_{n}, \\
\frac{3}{2}\left(\varepsilon_{1}+\varepsilon_{n+1}\right)+\sum_{j=2}^{n-1} 2 \varepsilon_{j}+\varepsilon_{n}, \\
2\left(\varepsilon_{1}+\varepsilon_{n+1}\right)+\sum_{j=2}^{n-1} 2 \varepsilon_{j} .
\end{gathered}
$$

Writing these $M$-types in terms of the $\tilde{\varepsilon}_{i}$ 's, we have

$$
\begin{array}{r}
3\left(\tilde{\varepsilon}_{1}-\tilde{\varepsilon}_{2}\right)+\sum_{j=3}^{n+1} 2 \tilde{\varepsilon}_{j}, \\
2\left(\tilde{\varepsilon}_{1}-\tilde{\varepsilon}_{2}\right)+\sum_{j=3}^{n+1} 2 \tilde{\varepsilon}_{j}, \\
\left(\tilde{\varepsilon}_{1}-\tilde{\varepsilon}_{2}\right)+\sum_{j=3}^{n+1} 2 \tilde{\varepsilon}_{j}, \\
\frac{5}{2}\left(\tilde{\varepsilon}_{1}-\tilde{\varepsilon}_{2}\right)+\sum_{j=3}^{n} 2 \tilde{\varepsilon}_{j}+\tilde{\varepsilon}_{n+1}, \\
\frac{3}{2}\left(\tilde{\varepsilon}_{1}-\tilde{\varepsilon}_{2}\right)+\sum_{j=3}^{n} 2 \tilde{\varepsilon}_{j}+\tilde{\varepsilon}_{n+1}, \\
2\left(\tilde{\varepsilon}_{1}-\tilde{\varepsilon}_{2}\right)+\sum_{j=3}^{n} 2 \tilde{\varepsilon}_{j} .
\end{array}
$$


Comparing these formulas with the ones giving the $\Lambda^{i j}$ 's, we have the assertion at the beginning of the proof. The $P^{i j+n}$ 's with $i+j+n=2 n$ are the ones for which the real root $\alpha$ is simple. Thus the result follows combining Corollaries 9.3 and 16.4 .

From now on we consider $G=\operatorname{Sp}(n, 1)$. Let $\xi \in \hat{M}$ be the irreducible representation of highest weight $\Lambda_{\xi}=b_{0}\left(\tilde{\varepsilon}_{1}-\tilde{\varepsilon}_{2}\right)+\sum_{j=3}^{n+1} b_{j} \tilde{\varepsilon}_{j}$ and $\nu \in a^{*}$. We need the following result of [8, Theorem $3(1)$, p. 24].

THEOREM 17.11. Let the representation $\pi_{\xi, \nu}$ be such that $b_{0}=0$ and $\frac{1}{2}(2(\nu, \alpha) /(\alpha, \alpha)+1) \in \mathbf{Z}$. Define $t_{j}=b_{j+2}+n-j+1$ for $j=2, \ldots, n$ and set $\nu=2(\nu, \alpha) /(\alpha, \alpha)$. Then if $(\nu+1) / 2>t_{2}+1$ or $t_{i-1}>(\nu+1) / 2>t_{i}+1, i=$ $3, \ldots, n$, the principal series has two invariant closed subspaces different from zero and from $H^{\xi, \nu}$.

We now want to prove that the discrete series $\pi_{n}$ of $\operatorname{Sp}(n, 1)$ is an irreducible component of $\pi_{n-2, n-1}$. We show, using opportune $K$-types, that the only Langlands quotient which may appear in the composition series of $\pi_{n-2, n-1}$, besides $J^{n-2, n-1}$, is $J^{n-2, n}$. (The $K$-types we consider are usually "lowest" in the sense of [16].) This fact together with Theorem 17.11 will give us the result.

The same fact in the case of $\operatorname{Sp}(2,1)$ follows directly by [9] where the composition series for the spherical principal series $\pi_{0,1}$ is described. For the notation we refer to everything set up to now.

Lemma 17.12. Let $\tau_{\mu_{1}}, 0 \leqslant i \leqslant n-1$, denote the irreducible $K$-module of highest weight $\mu_{i}$, where $\mu_{i}$ is defined in the following way:

$$
\begin{aligned}
\mu_{i} & =\sum_{j=1}^{i} 2 \varepsilon_{j}+\varepsilon_{i+1}+2\left(n-i-\frac{1}{2}\right) \varepsilon_{n+1}, \quad 0 \leqslant i \leqslant n-3, \\
\mu_{n-2} & =\sum_{j=1}^{n-1} 2 \varepsilon_{j}+4 \varepsilon_{n+1}, \\
\mu_{n-1} & =\sum_{j=1}^{n+1} 2 \varepsilon_{j} .
\end{aligned}
$$

Then $\tau_{\mu_{3}}$ is a $K$-type of $J^{i, 2 n-i}$ and of the following principal series:

$$
\pi_{i, 2 n-i-1}, \pi_{i, 2 n-i}, \pi_{i-1,2 n-i-1}, \pi_{i-1,2 n-i,} \quad 1 \leqslant i \leqslant n-1,
$$

and for $i=0$,

$$
\pi_{0,2 n-1}, \pi_{0,2 n}
$$

$\tau_{\mu_{i}}$ always has multiplicity one.

Proof. We compute the $M$-types of $\tau_{\mu_{i}}$. We first consider the case $0<i<n-3$. Then $\mu_{i}=\sum_{j=1}^{n+1} a_{j} \varepsilon_{j}$ with $a_{1}=\cdots=a_{i}=2, a_{i+1}=1, a_{i+2}=\cdots=a_{n}=0$ and $a_{n+1}=2\left(n-i-\frac{1}{2}\right)$.

We need to find all the $n$-tuples in $Z^{n}, b=\left(b_{1}, \ldots, b_{n}\right)$, satisfying (i)-(v) as in the proof of 12.4 . 
Let $b$ be an $n$-tuple of integers satisfying (i)-(iii). Then $b_{2}=\cdots=b_{i-1}=2$, $1 \leqslant b_{i} \leqslant 2, b_{i+1} \leqslant 2, b_{i+2} \leqslant 1$ and $b_{i+3}=\cdots=b_{n}=0$ (if $i=0, b_{2} \leqslant 1, b_{3}$ $=\cdots=b_{n}=0$ and if $i=1, b_{2} \leqslant 2, b_{3} \leqslant 1, b_{4}=\cdots=b_{n}=0$ ).

Also $A_{1}=\cdots=A_{i-1}=0, A_{i}=b_{i}-\max \left(1, b_{i+1}\right), A_{i+1}=\min \left(1, b_{i+1}\right)-b_{i+2}$, $A_{i+2}=\cdots=A_{n}=0$ (if $i=0, A_{1}=1-b_{2}, A_{2}=\cdots=A_{n}=0$, and if $i=1$, $\left.A_{1}=2-\max \left(1, b_{2}\right), A_{2}=\min \left(1, b_{2}\right)-b_{3}, A_{3}=\cdots=A_{n}=0\right) . \Sigma\left(a_{j}+b_{j}\right) 2 Z$ forces $b_{1}+b_{i}+b_{i+1}+b_{i+2}$ odd $\left(b_{1}+b_{2}\right.$ odd, if $i=0, b_{1}+b_{2}+b_{3}$ odd, if $i=1)$.

In the following table we give on the left a list of the possible $n$-tuples of integers satisfying (i)-(iv). For simplicity we only indicate the values of $b_{1}, b_{i}, b_{i+1}, b_{i+2} . b_{1}$ depends on a parameter $k \in \mathbf{Z}$, thus on the right we compute for which $k$ the $n$-tuple we are considering satisfies (v). We also give the value of $A_{i}, A_{i+1}$, and the multiplicity

$$
\begin{aligned}
& \tilde{m}_{\mu_{1}}(\mu)=\sum_{L \subset\{1, \ldots, n\}}(-1)^{|L|}\left(\begin{array}{c}
n-2-|L|+\frac{1}{2}\left(-b_{1}+\sum_{1}^{n} A_{i}\right)-\sum_{i \in L} A_{i} \\
n-2
\end{array}\right) \\
& \left(\mu=\sum_{i=1}^{n} b_{i} \varepsilon_{i}\right)
\end{aligned}
$$

and the value of $b_{0}=\left(a_{n+1}+b_{1}-2 j\right) / 2$, for $j=0, \ldots, \min \left(a_{n+1}, b_{1}\right)$.

$$
\begin{aligned}
& \begin{array}{lllllllll}
b_{1} & b_{i} & b_{i+1} & b_{i+2} & A_{i} & A_{i+1} & b_{1} & b_{0} & \tilde{m}_{\mu}(\mu)
\end{array} \\
& 0<i<n-3 \quad 2 k \quad 2 \quad 2 \quad 1 \quad 000000 \frac{a_{n+1}}{2} \\
& 0<i<n-3 \quad 2 k+1 \quad 2 \quad 2 \quad 0 \quad 0 \quad 1 \quad 1 \quad \frac{a_{n+1}+1}{2}, \frac{a_{n+1}-1}{2} \\
& 1<i<n-3 \quad 2 k+1 \quad 2 \quad 1 \quad 1 \quad 1 \quad 0 \quad 1 \quad \frac{a_{n+1}+1}{2}, \frac{a_{n+1}-1}{2} \\
& 1<i<n-3 \quad 2 k . \quad 2 \quad 1 \quad 0 \quad 1 \quad 1 \quad 0,2 \begin{cases}b_{1}=0: \frac{a_{n+1}}{2} & 1 \\
b_{1}=2: \frac{a_{n+1}+2}{2}, \frac{a_{n+1}-2}{2}, \frac{a_{n+1}}{2} & 1\end{cases} \\
& 1<i<n-3 \quad 2 k+1 \quad 2 \quad 0 \quad 0 \quad 1 \quad 0 \quad 1 \quad \frac{a_{n+1}+1}{2}, \frac{a_{n+1}-1}{2}
\end{aligned}
$$

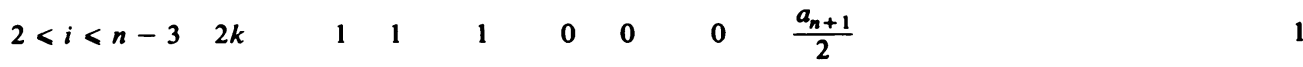

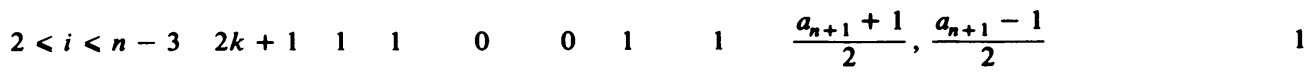

$$
\begin{aligned}
& 2<i<n-3 \quad 2 k \quad 1100 \quad 0 \quad 000000 \frac{a_{n+1}}{2}
\end{aligned}
$$

The computation is immediate but in the case of the $n$-tuple $b_{k}=\left(b_{1}, \ldots, b_{n}\right)$ where $b_{1}=2 k, b_{2}=\cdots=b_{i-1}=2, b_{i}=2, b_{i+1}=1, b_{i+2}=0, b_{i+3}=\cdots=b_{n}$ $=0$. We need to find for which $k, \tilde{m}_{\mu_{i}}\left(\mu_{b_{k}}\right) \neq 0$.

$$
\begin{aligned}
\tilde{m}_{\mu_{i}}\left(\mu_{b_{k}}\right) & =\sum_{L \subset\{1, \ldots, n\}}(-1)^{|L|}\left(\begin{array}{c}
n-2-|L|+\frac{1}{2}(-2 k+2)-\sum_{j \in L} A_{j} \\
n-2
\end{array}\right) \\
& =\sum_{\substack{L \subset\{1, \ldots, n\} \\
i, i+1 \notin L}}(-1)^{|L|}\left(\begin{array}{c}
n-2-|L|-k+1 \\
n-2
\end{array}\right) .
\end{aligned}
$$


If $k=0$ then

$$
\begin{aligned}
\tilde{m}_{\mu_{t}}\left(\mu_{b_{0}}\right) & =\sum_{\substack{L \subset\{1, \ldots, n\} \\
i, i+1 \notin L}}(-1)^{|L|}\left(\begin{array}{c}
n-2-|L|+1 \\
n-2
\end{array}\right) \\
& =\left(\begin{array}{c}
n-1 \\
n-2
\end{array}\right)-(n-2)\left(\begin{array}{l}
n-2 \\
n-2
\end{array}\right)=1 .
\end{aligned}
$$

If $k=1$ then $\tilde{m}_{\mu_{1}}\left(\mu_{b_{1}}\right)=1$. If $k=2$ then $\tilde{m}_{\mu_{i}}\left(\mu_{b_{k}}\right)=0$. Since $a_{n+1} \geqslant 2$, the values of $b_{0}$ are the ones indicated. Thus the $M$-types of $\tau_{\mu_{i}}, 0<i<n-3$, have highest weight relative to the $\tilde{\varepsilon}_{i}$ 's given by

$$
\begin{aligned}
& 0 \leqslant i \leqslant n-3, \quad \gamma_{1}^{i}=\left(n-i-\frac{1}{2}\right)\left(\tilde{\varepsilon}_{1}-\tilde{\varepsilon}_{2}\right)+\sum_{j=3}^{i} 2 \tilde{\varepsilon}_{j}+2 \tilde{\varepsilon}_{i+1}+2 \tilde{\varepsilon}_{i+2}+\tilde{\varepsilon}_{i+3} \\
& \text { with } m=m_{\mu_{i}}\left(\gamma_{1}^{i}\right)=1 \text {, } \\
& 0 \leqslant i \leqslant n-3, \quad \gamma_{2}^{i}=(n-i)\left(\tilde{\varepsilon}_{1}-\tilde{\varepsilon}_{2}\right)+\sum_{j=3}^{i} 2 \tilde{\varepsilon}_{j}+2 \tilde{\varepsilon}_{i+1}+2 \tilde{\varepsilon}_{i+2} \\
& \text { with } m=1 \text {, } \\
& 0 \leqslant i \leqslant n-3, \quad \gamma_{3}^{i}=(n-i-1)\left(\tilde{\varepsilon}_{1}-\tilde{\varepsilon}_{2}\right)+\sum_{j=3}^{i} 2 \tilde{\varepsilon}_{j}+2 \tilde{\varepsilon}_{i+1}+2 \tilde{\varepsilon}_{i+2} \\
& \text { with } m=1 \text {, } \\
& 1<i \leqslant n-3, \quad \gamma_{4}^{i}=(n-i)\left(\tilde{\varepsilon}_{1}-\tilde{\varepsilon}_{2}\right)+\sum_{j=3}^{i} 2 \tilde{\varepsilon}_{j}+2 \tilde{\varepsilon}_{i+1}+\tilde{\varepsilon}_{i+2}+\tilde{\varepsilon}_{i+3} \\
& \text { with } m=1 \text {, } \\
& 1<i \leqslant n-3, \quad \gamma_{5}^{i}=(n-i-1)\left(\tilde{\varepsilon}_{1}-\tilde{\varepsilon}_{2}\right)+\sum_{j=3}^{i} 2 \tilde{\varepsilon}_{j}+2 \tilde{\varepsilon}_{i+1}+\tilde{\varepsilon}_{i+2}+\tilde{\varepsilon}_{i+3} \\
& 1<i \leqslant n-3, \quad \gamma_{6}^{i}=\left(n-i-\frac{1}{2}\right)\left(\tilde{\varepsilon}_{1}-\tilde{\varepsilon}_{2}\right)+\sum_{j=3}^{i} 2 \tilde{\varepsilon}_{j}+2 \tilde{\varepsilon}_{i+1}+\tilde{\varepsilon}_{i+2} \\
& \text { with } m=1 \text {, } \\
& \text { with } m=2 \text {, } \\
& 1<i \leqslant n-3, \quad \gamma_{7}^{i}=\left(n-i+\frac{1}{2}\right)\left(\tilde{\varepsilon}_{1}-\tilde{\varepsilon}_{2}\right)+\sum_{j=3}^{i} 2 \tilde{\varepsilon}_{j}+2 \tilde{\varepsilon}_{i+1}+\tilde{\varepsilon}_{i+2} \\
& \text { with } m=1 \text {, } \\
& 1<i \leqslant n-3, \quad \gamma_{8}^{i}=\left(n-i-\frac{3}{2}\right)\left(\tilde{\varepsilon}_{1}-\tilde{\varepsilon}_{2}\right)+\sum_{j=3}^{i} 2 \tilde{\varepsilon}_{j}+2 \tilde{\varepsilon}_{i+1}+\tilde{\varepsilon}_{i+2} \\
& \text { with } m=1 \text {, } \\
& 1<i \leqslant n-3, \quad \gamma_{9}^{i}=(n-i)\left(\tilde{\varepsilon}_{1}-\tilde{\varepsilon}_{2}\right)+\sum_{j=3}^{i} 2 \tilde{\varepsilon}_{j}+2 \tilde{\varepsilon}_{i+1} \quad \text { with } m=1 \text {, } \\
& 1<i<n-3, \quad \gamma_{10}^{i}=(n-i-1)\left(\tilde{\varepsilon}_{1}-\tilde{\varepsilon}_{2}\right)+\sum_{j=3}^{i} 2 \tilde{\varepsilon}_{j}+2 \tilde{\varepsilon}_{i+1} \quad \text { with } m=1,
\end{aligned}
$$




$$
\begin{array}{lll}
2 \leqslant i \leqslant n-3, & \gamma_{11}^{i}=\left(n-i-\frac{1}{2}\right)\left(\tilde{\varepsilon}_{1}-\tilde{\varepsilon}_{2}\right)+\sum_{j=3}^{i} 2 \tilde{\varepsilon}_{j}+\tilde{\varepsilon}_{i+1}+\tilde{\varepsilon}_{i+2}+\tilde{\varepsilon}_{i+3} \\
2 \leqslant i \leqslant n-3, & \gamma_{12}^{i}=(n-i)\left(\tilde{\varepsilon}_{1}-\tilde{\varepsilon}_{2}\right)+\sum_{j=3}^{i} 2 \tilde{\varepsilon}_{j}+\tilde{\varepsilon}_{i+1}+\tilde{\varepsilon}_{i+2} \quad \text { with } m=1, & \text { with } m=1, \\
2 \leqslant i \leqslant n-3, & \gamma_{13}^{i}=(n-i-1)\left(\tilde{\varepsilon}_{1}-\tilde{\varepsilon}_{2}\right)+\sum_{j=3}^{i} 2 \tilde{\varepsilon}_{j}+\tilde{\varepsilon}_{i+1}+\tilde{\varepsilon}_{i+2} & \text { with } m=1, \\
2 \leqslant i \leqslant n-3, & \gamma_{14}^{i}=\left(n-i-\frac{1}{2}\right)\left(\tilde{\varepsilon}_{1}-\tilde{\varepsilon}_{2}\right)+\sum_{j=3}^{i} 2 \tilde{\varepsilon}_{j}+\tilde{\varepsilon}_{i+1} \quad \text { with } m=1 .
\end{array}
$$

To prove the second assertion of the lemma by the Frobenius reciprocity theorem, we have just to check which of the $M$-types we just found is equal to the $M$-types described by the $\Lambda_{i, j}$ 's or the $\Lambda_{i j+n}$ 's. The computation is straightforward and we omit it (see f.i. Lemma 15.8).

(a) $\tau_{\mu_{i}}$ is not a $K$-type of $\left(\pi_{i}, H_{F}^{i}\right)$.

Indeed $\mu_{i}=2 \delta_{n}^{C_{i}}-\left(\varepsilon_{n+1}-\varepsilon_{i+1}\right)$ with $\varepsilon_{n+1}-\varepsilon_{i+1}$ simple for $C_{i}$. Hence (a) follows by [15].

(b) $\tau_{\mu_{i}}$ is not a $K$-type of $\left(\pi_{i+1}, H_{F}^{i+1}\right)$.

Indeed $\mu_{i}=2 \delta_{n}^{C_{i+1}}-\left(\varepsilon_{i+1}-\varepsilon_{n+1}\right)$ with $\varepsilon_{i+1}-\varepsilon_{n+1}$ simple for $C_{i+1}$. Again (b) follows by [15].

By Lemma 15.8 and Corollary 16.4 we know that $\pi_{i}, \pi_{i+1}$ are subspaces of $\pi_{i, 2 n-i}$ and that they are the only discrete series which are irreducible components of $\pi_{i, 2 n-i}$. Thus by Theorem 17.4 we have that the composition series of $\pi_{i, 2 n-i}$ is given by $H_{F}^{i, 2 n-i} \supset H_{F}^{i} \oplus H_{F}^{i+1} \supset H_{F}^{i} \supset(0)$.

Using (a) and (b) we may conclude that $\tau_{\mu_{4}}$ is a $K$-type of $J^{i, 2 n-i}$ as asserted.

The proofs for $\tau_{\mu_{n-2}}$ and $\tau_{p_{n-1}}$ are very similar and we omit them.

LEMMA 17.13. Let $\tau_{\beta_{i}}, 0 \leqslant i \leqslant n-1$, denote the irreducible $K$-module of highest weight $\beta_{i}$, where $\beta_{i}$ is defined in the following way:

$$
\begin{aligned}
\beta_{i} & =2 \sum_{j=1}^{i} \varepsilon_{j}+\varepsilon_{i+1}+\varepsilon_{i+2}+2(n-i-1) \varepsilon_{n+1}, \quad 0 \leqslant i \leqslant n-2, \\
\beta_{n-1} & =2 \sum_{j=1}^{n-1} \varepsilon_{j} .
\end{aligned}
$$

Then $\tau_{\beta_{i}}$ is a $K$-type of $J^{i, 2 n-i-1}$ and of the following principal series:

$$
\begin{array}{ll}
1 \leqslant i \leqslant n-4, & \pi_{i, 2 n-i-2}, \pi_{i, 2 n-i-1}, \pi_{i-1,2 n-i-2}, \pi_{i-1,2 n-i-1}, \\
i=0, & \pi_{0,2 n-2}, \pi_{0,2 n-1}, \\
i=n-2, & \pi_{n-2, n}, \pi_{n-2, n+1}, \pi_{n-3, n-1}, \pi_{n-3, n}, \pi_{n-3, n+1}, \pi_{n-4, n-1}, \pi_{n-4, n}, \\
i=n-3, & \pi_{n-3, n+1}, \pi_{n-3, n+2}, \pi_{n-4, n-1}, \pi_{n-4, n}, \pi_{n-4, n+1}, \pi_{n-4, n+2}, \\
i=n-1, & \pi_{n-1, n}, \pi_{n-2, n}, \pi_{n-3, n-2}, \pi_{n-3, n-1} .
\end{array}
$$


Proof. The $M$-types of $\tau_{\beta_{i}}, 0 \leqslant i \leqslant n-2$, have highest weight relative to the $\tilde{\varepsilon}_{i}$ 's given by

$0<i \leqslant n-3, \quad \gamma_{1}^{i}=(n-i-1)\left(\tilde{\varepsilon}_{1}-\tilde{\varepsilon}_{2}\right)$

$$
+\sum_{j=3}^{i} 2 \tilde{\varepsilon}_{j}+2 \tilde{\varepsilon}_{i+1}+\tilde{\varepsilon}_{i+2}+\tilde{\varepsilon}_{i+3}+\tilde{\varepsilon}_{i+4} \quad \text { with } m=1
$$

$0 \leqslant i \leqslant n-2, \quad \gamma_{2}^{i}=\left(n-1-\frac{1}{2}\right)\left(\tilde{\varepsilon}_{1}-\tilde{\varepsilon}_{2}\right)+\sum_{j=3}^{i} 2 \tilde{\varepsilon}_{j}+2 \tilde{\varepsilon}_{i+1}+2 \tilde{\varepsilon}_{i+2}+\tilde{\varepsilon}_{i+3}$

with $m=1$,

$0 \leqslant i \leqslant n-2, \quad \gamma_{3}^{i}=\left(n-i-\frac{3}{2}\right)\left(\tilde{\varepsilon}_{1}-\tilde{\varepsilon}_{2}\right)+\sum_{j=3}^{i} 2 \tilde{\varepsilon}_{j}+2 \tilde{\varepsilon}_{i+1}+2 \tilde{\varepsilon}_{i+2}+\tilde{\varepsilon}_{i+3}$

with $m=1$,

$0 \leqslant i \leqslant n-2, \quad \gamma_{4}^{i}=(n-i-1)\left(\tilde{\varepsilon}_{1}-\tilde{\varepsilon}_{2}\right)+\sum_{j=3}^{i} 2 \tilde{\varepsilon}_{j}+2 \tilde{\varepsilon}_{i+1}+2 \tilde{\varepsilon}_{i+2}$

$1 \leqslant i \leqslant n-3, \quad \gamma_{5}^{i}=\left(n-i-\frac{1}{2}\right)\left(\tilde{\varepsilon}_{1}-\tilde{\varepsilon}_{2}\right)$

with $m=1$,

$$
+\sum_{j=3}^{i} 2 \tilde{\varepsilon}_{j}+2 \tilde{\varepsilon}_{i+1}+\tilde{\varepsilon}_{i+2}+\tilde{\varepsilon}_{i+3}+\tilde{\varepsilon}_{i+4} \quad \text { with } m=1,
$$

$1 \leqslant i \leqslant n-3, \quad \gamma_{6}^{i}=\left(n-i-\frac{3}{2}\right)\left(\tilde{\varepsilon}_{1}-\tilde{\varepsilon}_{2}\right)$

$$
+\sum_{j=3}^{i} 2 \tilde{\varepsilon}_{j}+2 \tilde{\varepsilon}_{i+1}+\tilde{\varepsilon}_{i+2}+\tilde{\varepsilon}_{i+3}+\tilde{\varepsilon}_{i+4} \quad \text { with } m=1,
$$

$1 \leqslant i \leqslant n-2, \quad \gamma_{7}^{i}=(n-i-1)\left(\tilde{\varepsilon}_{1}-\tilde{\varepsilon}_{2}\right)+\sum_{j=3}^{i} 2 \tilde{\varepsilon}_{j}+2 \tilde{\varepsilon}_{i+1}+\tilde{\varepsilon}_{i+2}+\tilde{\varepsilon}_{i+3}$

with $m=2$,

$1 \leqslant i \leqslant n-2, \quad \gamma_{8}^{i}=(n-i)\left(\tilde{\varepsilon}_{1}-\tilde{\varepsilon}_{2}\right)+\sum_{j=3}^{i} 2 \tilde{\varepsilon}_{j}+2 \tilde{\varepsilon}_{i+1}+\tilde{\varepsilon}_{i+2}+\tilde{\varepsilon}_{i+3}$

$$
\text { with } m=1 \text {, }
$$

$1 \leqslant i \leqslant n-2, \quad \gamma_{9}^{i}=(n-i-2)\left(\tilde{\varepsilon}_{1}-\tilde{\varepsilon}_{2}\right)+\sum_{j=3}^{i} 2 \tilde{\varepsilon}_{j}+2 \tilde{\varepsilon}_{i+1}+\tilde{\varepsilon}_{i+2}+\tilde{\varepsilon}_{i+3}$

$$
\text { with } m=1 \text {, }
$$

$1 \leqslant i \leqslant n-2, \quad \gamma_{10}^{i}=\left(n-i-\frac{1}{2}\right)\left(\tilde{\varepsilon}_{1}-\tilde{\varepsilon}_{2}\right)+\sum_{j=3}^{i} 2 \tilde{\varepsilon}_{j}+2 \tilde{\varepsilon}_{i+1}+\tilde{\varepsilon}_{i+2}$

with $m=1$, 
$1 \leqslant i \leqslant n-2, \quad \gamma_{11}^{i}=\left(n-i-\frac{3}{2}\right)\left(\tilde{\varepsilon}_{1}-\tilde{\varepsilon}_{2}\right)+\sum_{j=3}^{i} 2 \tilde{\varepsilon}_{j}+2 \tilde{\varepsilon}_{i+1}+\tilde{\varepsilon}_{i+2}$

with $m=1$,

$2 \leqslant i \leqslant n-3, \quad \gamma_{12}^{i}=(n-i-1)\left(\tilde{\varepsilon}_{1}-\tilde{\varepsilon}_{2}\right)+\sum_{j=3}^{i} 2 \tilde{\varepsilon}_{j}+\tilde{\varepsilon}_{i+1}+\tilde{\varepsilon}_{i+2}+\tilde{\varepsilon}_{i+3}+\tilde{\varepsilon}_{i+4}$ with $m=1$,

$2 \leqslant i \leqslant n-2, \quad \gamma_{13}^{i}=\left(n-i-\frac{1}{2}\right)\left(\tilde{\varepsilon}_{1}-\tilde{\varepsilon}_{2}\right)+\sum_{j=3}^{i} 2 \tilde{\varepsilon}_{j}+\tilde{\varepsilon}_{i+1}+\tilde{\varepsilon}_{i+2}+\tilde{\varepsilon}_{i+3}$

with $m=1$,

$2 \leqslant i \leqslant n-2, \quad \gamma_{14}^{i}=\left(n-i-\frac{3}{2}\right)\left(\tilde{\varepsilon}_{1}-\tilde{\varepsilon}_{2}\right)+\sum_{j=3}^{i} 2 \tilde{\varepsilon}_{j}+\tilde{\varepsilon}_{i+1}+\tilde{\varepsilon}_{i+2}+\tilde{\varepsilon}_{i+3}$

$$
\text { with } m=1 \text {, }
$$

$2 \leqslant i \leqslant n-2, \quad \gamma_{15}^{i}=(n-i-1)\left(\tilde{\varepsilon}_{1}-\tilde{\varepsilon}_{2}\right)+\sum_{j=3}^{i} 2 \tilde{\varepsilon}_{j}+\tilde{\varepsilon}_{i+1}+\tilde{\varepsilon}_{i+2} \quad$ with $m=1$.

The computation is very similar to the one of the previous lemma, hence we omit it. To prove the second assertion of the lemma by the Frobenius reciprocity theorem, we have just to check which of the $M$-types in the previous list is equal to the $M$-types described by the $\Lambda_{i, j}$ 's or the $\Lambda_{i, j+n}$ 's. Again we omit the computation.

Hence $\tau_{\beta_{i}}$ is a $K$-type of the described principal series. The only irreducible components of $\pi_{i, 2 n-i-1}, 0 \leqslant i \leqslant n-2$, are $\pi_{i+1}, J^{i, 2 n-i-1}$ and eventually a Langlands quotient of the form $J^{k, 2 n-k}$ for some $0 \leqslant k \leqslant n-1$ (cf. Theorem 17.4).

Hence $\tau_{\beta_{i}}$ must be a $K$-type of $J^{i, 2 n-i-1}$, otherwise it would be either a $K$-type of $\pi_{i+1}$ or possibly of $J^{k, 2 n-k}$ for some $k$ (this is impossible because then $\tau_{\beta_{i}}$ would be a $K$-type of $\pi_{i+1,2 n-i-1}$ or of $\pi_{k, 2 n-k}$ ).

The proof for $\tau_{\beta_{n-1}}$ is very similar and we omit it.

LEMMA 17.14. Let $\tau_{\gamma_{i}}, 0 \leqslant i \leqslant n-3$, be the irreducible $K$-module of highest weight $\gamma_{i}$, where $\gamma_{i}$ is defined in the following way:

$$
\gamma_{i}=\sum_{j=1}^{i} 2 \varepsilon_{j}+\varepsilon_{i+1}+\varepsilon_{i+2}+\varepsilon_{i+3}+2\left(n-i-\frac{3}{2}\right) \varepsilon_{n+1} .
$$

Then $\tau_{\gamma_{i}}$ is a $K$-type of $J^{i, 2 n-i-2}$ and of the following principal series:

$$
\begin{array}{ll}
1 \leqslant i \leqslant n-4, & \pi_{i, 2 n-i-3}, \pi_{i, 2 n-i-2}, \pi_{i-1,2 n-i-3}, \pi_{i-1,2 n-i-2}, \\
i=0, & \pi_{0,2 n-3}, \pi_{0,2 n-2}, \\
i=n-3, & \pi_{n-3, n}, \pi_{n-3, n+1}, \pi_{n-4, n-1}, \pi_{n-4, n}, \pi_{n-4, n+1}, \pi_{n-5, n-1}, \pi_{n-5, n}, \\
i=n-4, & \pi_{n-4, n+1}, \pi_{n-4, n+2}, \pi_{n-5, n-1}, \pi_{n-5, n}, \pi_{n-5, n+1}, \pi_{n-5, n+2}, \\
&
\end{array}
$$

$\tau_{\gamma_{i}}$ always has multiplicity one, but $\tau_{\gamma_{n-3}}$ has multiplicity two in $\pi_{n-4, n}$.

Proof. We omit the proof because it involves the same computational details of the two previous lemmas. We only give a list of the $M$-types for the convenience of the reader. 
The $M$-types of $\tau_{\gamma_{i}}, 0 \leqslant i \leqslant n-3$, have highest weight:

$$
\begin{aligned}
0 \leqslant i \leqslant n-4, \quad \beta_{1}^{i}= & \left(n-i-\frac{3}{2}\right)\left(\tilde{\varepsilon}_{1}-\tilde{\varepsilon}_{2}\right) \\
& +\sum_{3}^{i} 2 \tilde{\varepsilon}_{i}+2 \tilde{\varepsilon}_{i+1}+2 \tilde{\varepsilon}_{i+2}+\tilde{\varepsilon}_{i+3}+\tilde{\varepsilon}_{i+4}+\tilde{\varepsilon}_{i+5},
\end{aligned}
$$

$0 \leqslant i \leqslant n-3, \quad \beta_{2}^{i}=(n-i-1)\left(\tilde{\varepsilon}_{1}-\tilde{\varepsilon}_{2}\right)$

$$
+\sum_{3}^{i} 2 \tilde{\varepsilon}_{i}+2 \tilde{\varepsilon}_{i+1}+2 \tilde{\varepsilon}_{i+2}+\tilde{\varepsilon}_{i+3}+\tilde{\varepsilon}_{i+4}
$$

$0 \leqslant i \leqslant n-3, \quad \beta_{3}^{i}=(n-i-2)\left(\tilde{\varepsilon}_{1}-\tilde{\varepsilon}_{2}\right)$

$$
+\sum_{3}^{i} 2 \tilde{\varepsilon}_{i}+2 \tilde{\varepsilon}_{i+1}+2 \tilde{\varepsilon}_{i+2}+\tilde{\varepsilon}_{i+3}+\tilde{\varepsilon}_{i+4}
$$

$0 \leqslant i \leqslant n-3, \quad \beta_{4}^{i}=\left(n-i-\frac{3}{2}\right)\left(\tilde{\varepsilon}_{1}-\tilde{\varepsilon}_{2}\right)+\sum_{3}^{i} 2 \tilde{\varepsilon}_{i}+2 \tilde{\varepsilon}_{i+1}+2 \tilde{\varepsilon}_{i+2}+\tilde{\varepsilon}_{i+3}$,

$1 \leqslant i \leqslant n-4, \quad \beta_{5}^{i}=(n-i-1)\left(\tilde{\varepsilon}_{1}-\tilde{\varepsilon}_{2}\right)$

$$
+\sum_{3}^{i} 2 \tilde{\varepsilon}_{i}+2 \tilde{\varepsilon}_{i+1}+\tilde{\varepsilon}_{i+2}+\tilde{\varepsilon}_{i+3}+\tilde{\varepsilon}_{i+4}+\tilde{\varepsilon}_{i+5}
$$

$1 \leqslant i \leqslant n-4, \quad \beta_{6}^{i}=(n-i-2)\left(\tilde{\varepsilon}_{1}-\tilde{\varepsilon}_{2}\right)$

$$
+\sum_{3}^{i} 2 \tilde{\varepsilon}_{i}+2 \tilde{\varepsilon}_{i+1}+\tilde{\varepsilon}_{i+2}+\tilde{\varepsilon}_{i+3}+\tilde{\varepsilon}_{i+4}+\tilde{\varepsilon}_{i+5}
$$

$1 \leqslant i \leqslant n-3, \quad \beta_{7}^{i}=\left(n-i-\frac{3}{2}\right)\left(\tilde{\varepsilon}_{1}-\tilde{\varepsilon}_{2}\right)$

$$
+\sum_{3}^{i} 2 \tilde{\varepsilon}_{i}+2 \tilde{\varepsilon}_{i+1}+\tilde{\varepsilon}_{i+2}+\tilde{\varepsilon}_{i+3}+\tilde{\varepsilon}_{i+4}
$$

$1 \leqslant i \leqslant n-3, \quad \beta_{8}^{i}=\left(n-i-\frac{1}{2}\right)\left(\tilde{\varepsilon}_{1}-\tilde{\varepsilon}_{2}\right)$

$$
+\sum_{3}^{1} 2 \tilde{\varepsilon}_{i}+2 \tilde{\varepsilon}_{i+1}+\tilde{\varepsilon}_{i+2}+\tilde{\varepsilon}_{i+3}+\tilde{\varepsilon}_{i+4}
$$

$1 \leqslant i \leqslant n-3, \quad \beta_{9}^{i}=\left(n-i-\frac{5}{2}\right)\left(\tilde{\varepsilon}_{1}-\tilde{\varepsilon}_{2}\right)$

$$
+\sum_{3}^{1} 2 \tilde{\varepsilon}_{i}+2 \tilde{\varepsilon}_{i+1}+\tilde{\varepsilon}_{i+2}+\tilde{\varepsilon}_{i+3}+\tilde{\varepsilon}_{i+4}
$$

$1 \leqslant i \leqslant n-3, \quad \beta_{10}^{i}=(n-i-1)\left(\tilde{\varepsilon}_{1}-\tilde{\varepsilon}_{2}\right)+\sum_{3}^{1} 2 \tilde{\varepsilon}_{i}+2 \tilde{\varepsilon}_{i+1}+\tilde{\varepsilon}_{i+2}+\tilde{\varepsilon}_{i+3}$,

$1 \leqslant i \leqslant n-3, \quad \beta_{11}^{i}=(n-i-2)\left(\tilde{\varepsilon}_{1}-\tilde{\varepsilon}_{2}\right)+\sum_{3}^{1} 2 \tilde{\varepsilon}_{i}+2 \tilde{\varepsilon}_{i+1}+\tilde{\varepsilon}_{i+2}+\tilde{\varepsilon}_{i+3}$, 


$$
\begin{array}{ll}
2 \leqslant i \leqslant n-4, \quad \beta_{12}^{i}= & \left(n-i-\frac{3}{2}\right)\left(\tilde{\varepsilon}_{1}-\tilde{\varepsilon}_{2}\right) \\
& +\sum_{3}^{1} 2 \tilde{\varepsilon}_{i}+\tilde{\varepsilon}_{i+1}+\tilde{\varepsilon}_{i+2}+\tilde{\varepsilon}_{i+3}+\tilde{\varepsilon}_{i+4}+\tilde{\varepsilon}_{i+5}, \\
2 \leqslant i \leqslant n-3, \quad \beta_{13}^{i}=(n-i-1)\left(\tilde{\varepsilon}_{1}-\tilde{\varepsilon}_{2}\right)+\sum_{3}^{1} 2 \tilde{\varepsilon}_{i}+\tilde{\varepsilon}_{i+1}+\tilde{\varepsilon}_{i+2}+\tilde{\varepsilon}_{i+3}+\tilde{\varepsilon}_{i+4}, \\
2 \leqslant i \leqslant n-3, \quad \beta_{14}^{i}=(n-i-2)\left(\tilde{\varepsilon}_{1}-\tilde{\varepsilon}_{2}\right)+\sum_{3}^{1} 2 \tilde{\varepsilon}_{i}+\tilde{\varepsilon}_{i+1}+\tilde{\varepsilon}_{i+2}+\tilde{\varepsilon}_{i+3}+\tilde{\varepsilon}_{i+4}, \\
2 \leqslant i \leqslant n-3, \quad \beta_{15}^{i}=\left(n-i-\frac{3}{2}\right)\left(\tilde{\varepsilon}_{1}-\tilde{\varepsilon}_{2}\right)+\sum_{3}^{1} 2 \tilde{\varepsilon}_{i}+\tilde{\varepsilon}_{i+1}+\tilde{\varepsilon}_{i+2}+\tilde{\varepsilon}_{i+3} .
\end{array}
$$

They all have multiplicity one, but $\beta_{7}^{i}$ has multiplicity 2 . Once the second assertion of the lemma is proved, since there are no discrete series in $\pi_{i, 2 n-i-2}, 0<i<n-$ 3 , then $\tau_{\gamma_{i}}$ must be a $K$-type of $J^{i, 2 n-i}$ by Theorem 17.4.

LEMMA 17.15. Let $\tau_{\alpha}$ denote the irreducible $K$-module of highest weight $\sum_{i=1}^{n-2} 2 \varepsilon_{i}+$ $\varepsilon_{n-1}+\varepsilon_{n}$. Then $\tau_{\alpha}$ is a $K$-type of $J^{n-2, n}$ and of the following principal series: $\pi_{n-2, n-1}, \pi_{n-2, n}, \pi_{n-3, n-1}, \pi_{n-3, n} . \tau_{\alpha}$ always has multiplicity one.

Proof. The $M$-types of $\tau_{\alpha}$ have highest weights:

$$
\begin{aligned}
& \alpha_{1}=\frac{1}{2}\left(\tilde{\varepsilon}_{1}-\tilde{\varepsilon}_{2}\right)+\sum_{j=3}^{n-2} 2 \tilde{\varepsilon}_{j}+2 \tilde{\varepsilon}_{n-1}+2 \tilde{\varepsilon}_{n}+\tilde{\varepsilon}_{n+1}, \\
& \alpha_{2}=\sum_{j=3}^{n-2} 2 \tilde{\varepsilon}_{j}+2 \tilde{\varepsilon}_{n-1}+2 \tilde{\varepsilon}_{n}, \\
& \alpha_{3}=\sum_{j=3}^{n-2} 2 \tilde{\varepsilon}_{j}+2 \tilde{\varepsilon}_{n-1}+\tilde{\varepsilon}_{n}+\tilde{\varepsilon}_{n+1}, \\
& \alpha_{4}=\left(\tilde{\varepsilon}_{1}-\tilde{\varepsilon}_{2}\right)+\sum_{j=3}^{n-2} 2 \tilde{\varepsilon}_{j}+2 \tilde{\varepsilon}_{n-1}+\tilde{\varepsilon}_{n}+\tilde{\varepsilon}_{n+1}, \\
& \alpha_{5}=\frac{1}{2}\left(\tilde{\varepsilon}_{1}-\tilde{\varepsilon}_{2}\right)+\sum_{j=3}^{n-2} 2 \tilde{\varepsilon}_{j}+2 \tilde{\varepsilon}_{n-1}+\tilde{\varepsilon}_{n}, \\
& \alpha_{6}=\frac{1}{2}\left(\tilde{\varepsilon}_{1}-\tilde{\varepsilon}_{2}\right)+\sum_{j=3}^{n-2} 2 \tilde{\varepsilon}_{j}+\tilde{\varepsilon}_{n-1}+\tilde{\varepsilon}_{n}+\tilde{\varepsilon}_{n+1}, \\
& \alpha_{7}=\sum_{j=3}^{n-2} 2 \tilde{\varepsilon}_{j}+\tilde{\varepsilon}_{n-1}+\tilde{\varepsilon}_{n} .
\end{aligned}
$$

Once the second assertion of the lemma is proved, since there are no discrete series in $\pi_{n-2, n}$, then $\tau_{\alpha}$ is a $K$-type of $J^{n, n-2}$ by Theorem 17.4.

THEOREM 17.16. (1) $\pi_{n-2, n-1}$ has a composition series of length three; the irreducible components are: $J^{n-2, n-1}, J^{n-2, n}$ and the discrete series $\pi_{n}$.

(2) $\pi_{n}$ is an irreducible component of $\pi_{n-2, n}$. 
Proof. The parameters which describe the principal series $\pi_{n-2, n-1}$ are

$$
\begin{aligned}
\Lambda_{n-2, n-1_{1 b^{-}}}=b_{0}\left(\tilde{\varepsilon}_{1}-\tilde{\varepsilon}_{2}\right)+ & \sum_{j=3}^{n+1} b_{j} \tilde{\varepsilon}_{j} \\
& \text { with } b_{0}=0, b_{j}=2 \text {, for } 3 \leqslant j \leqslant n \text { and } b_{n+1}=0,
\end{aligned}
$$

and

$$
\nu=\nu_{n-2, n-1} \quad \text { with } 2(\nu, \alpha) /(\alpha, \alpha)=5 .
$$

Thus $\frac{1}{2}(2(\nu, \alpha) /(\alpha, \alpha)+1)=3$.

Let $t_{j}$ be as in Theorem 17.11, then $t_{j}=n-j+3,2 \leqslant j \leqslant n-1 ; t_{n}=1$ and $4=t_{n-1}>\frac{1}{2}(2(\nu, \alpha) /(\alpha, \alpha)+1)>t_{n}+1=2$.

Hence by Theorem 17.11, we can conclude that the composition series of $\pi_{n-2, n-1}$ has length $\geqslant$ three.

On the other hand we know that the only irreducible components of $\pi_{n-2, n-1}$, could be, besides $J^{n-2, n-1}$, the discrete series $\pi_{n}$ (cf. Lemma 15.8) or the Langlands quotient $J^{n-2, n}$ (combine Lemmas 17.12, 17.13, 17.14, 17.15 and Theorem 17.4). Therefore ( 1 ) is proved.

$\pi_{n}$ may be an irreducible component of $\pi_{n-3, n-1}, \pi_{n-2, n}$ and $\pi_{n-1, n+1}$ (Lemma 15.8). Since $\pi_{n}$ is in $\pi_{n-1, n+1}$ (Lemma 16.6) then (2) is a consequence of (1) and of Corollary 9.3 .

18. Tables. We maintain the notation of $\$ \S 10-17$.

Let $Q$ be positive for $\Delta$ and containing $\Delta_{k}^{+}$. The following tables will give realizations of the discrete series $\pi_{\delta_{Q}}$ in the principal series for which the a parameter is real and in the positive Weyl chamber for a. As we saw in the discussion of 11.1, this is enough for our purpose.

Because of the minimal $K$-type Theorem 5.1 and Lemma 7.1, the parameters of the principal series where $\pi_{\delta_{Q}}$ may appear are the ones computed in $\S 15$.

We use the following symbols:

- the discrete series appears as a subspace,

$\otimes \quad$ the discrete series appears, and it is not a quotient.

If none of the symbols appear, then no discrete series occurs.

\section{TABLE I. $\operatorname{Spin}(2 n, 1), n \geqslant 2$}

Let $\pi_{1}, \pi_{2}$ be the discrete series representations associated with $\delta_{B_{1}}, \delta_{B_{2}}$ (cf. Lemma 11.5).

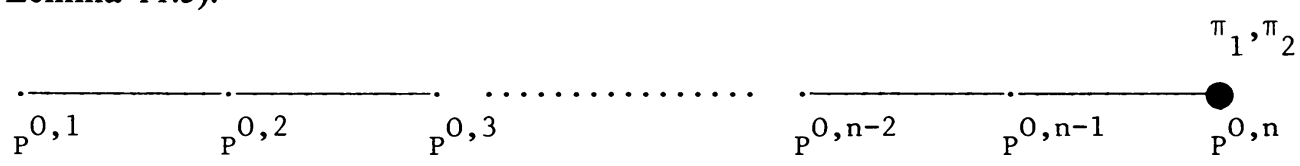

The parameters of the principal series $\pi_{\Lambda_{0, j_{b} b}, \nu_{0, j}}(0 \leqslant j \leqslant n)$ associated with $P^{0, j}$ are

$$
\Lambda_{0, j_{1 b^{-}}}=\sum_{k=2} \tilde{\varepsilon}_{k} \frac{2\left(\nu_{0, j}, \alpha\right)}{(\alpha, \alpha)}=2\left(n-j+\frac{1}{2}\right) \text {. }
$$

Comments. ${ }^{-}$is because of Lemma 11.6. 
TABLE II. $\mathrm{SU}(n, 1), n \geqslant 2$

Let $\pi_{i}(0 \leqslant i \leqslant n)$, be the discrete series representation parametrized by $\delta_{A_{i}}$ (cf. Lemma 11.6).

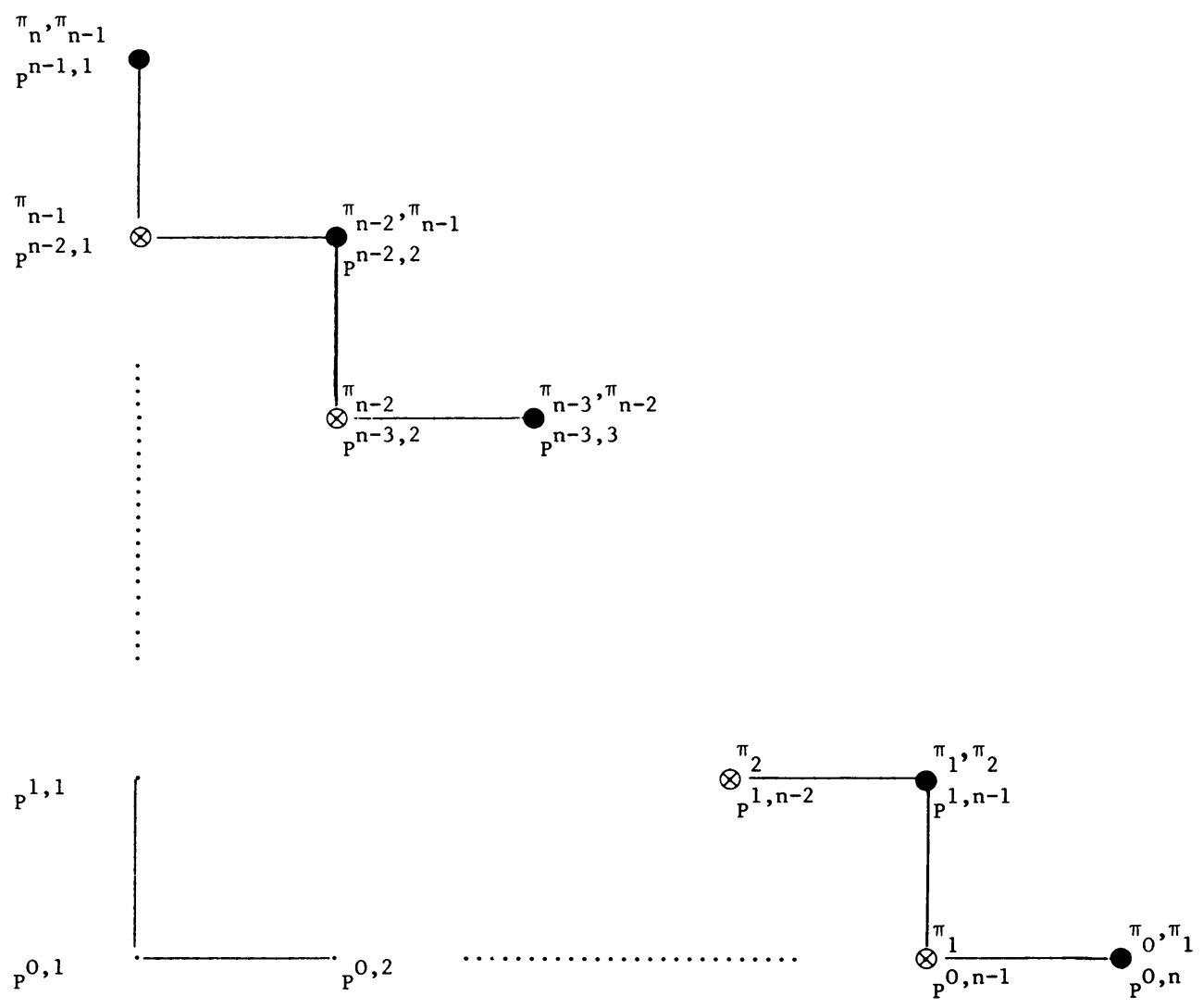

The parameters of the principal series $\pi_{\Lambda_{i, j \mid b^{-}}, \nu_{i, j}}(0<i<n-1,1<j<n-i)$, associated to $P^{i, j}$ are

$$
\begin{aligned}
\Lambda_{i, j_{\mid \varphi^{-}}} & =\frac{j-i-1}{2}\left(\tilde{\varepsilon}_{1}+\tilde{\varepsilon}_{n+1}\right)+\sum_{k=2}^{i+1} \tilde{\varepsilon}_{k}+\sum_{k=n+2-j}^{n} \tilde{\varepsilon}_{k} \text { and } \\
\frac{2\left(\nu_{i, j}, \alpha\right)}{(\alpha, \alpha)} & =n-j-i+1, \quad \alpha=\tilde{\varepsilon}_{1}-\tilde{\varepsilon}_{n+1} .
\end{aligned}
$$

Comments. Lemma 16.2 guarantees two realizations of $\pi_{i}, 1<i<n-1$, as a subspace and one for $\pi_{0}$ and $\pi_{n}$. On the other hand the $P^{i, j}$ marked with ${ }^{\bullet}$ are just the ones for which $\alpha$ is simple. Thus apply Lemma 16.6. Finally Corollary 9.3 gives the rest. (See Table III.)

The parameters of the principal series $\pi_{\Lambda_{i, j},-, \nu_{i j}} 0<i<j<n,\left(\pi_{\Lambda_{i, j b}, \nu_{i j+n}}, 0<i\right.$ $\leqslant n-1,1 \leqslant j \leqslant n-i)$ associated with $P^{i, j}\left(P^{i, j+n}\right)$ are 

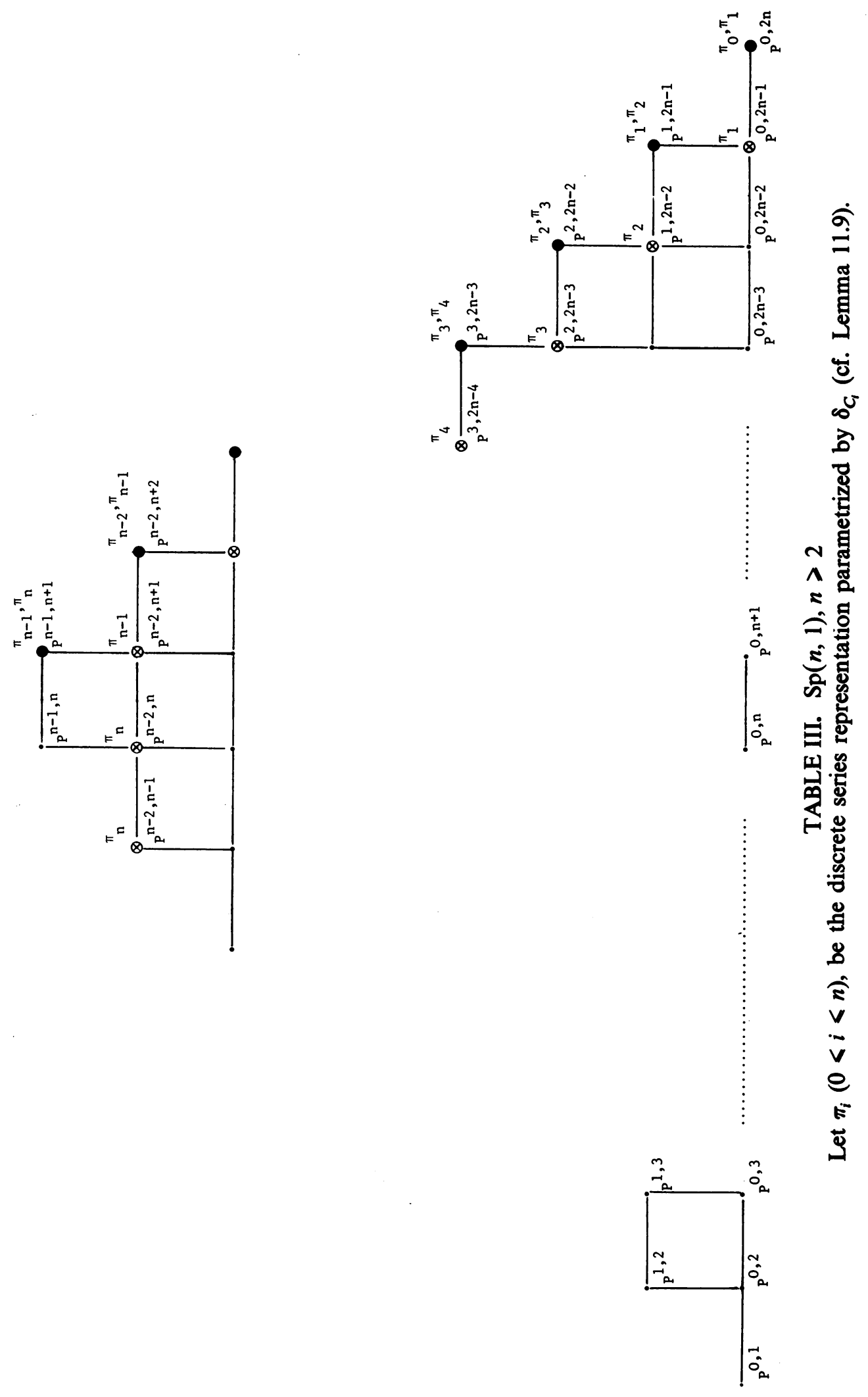


$$
\begin{aligned}
\Lambda_{i, j_{\mid h^{-}}} & =\frac{-i+j-1}{2}\left(\tilde{\varepsilon}_{1}-\tilde{\varepsilon}_{2}\right)+2 \sum_{k=3}^{i+2} \tilde{\varepsilon}_{k}+\sum_{k=i+3}^{j+1} \tilde{\varepsilon}_{k}, \\
\frac{2\left(\nu_{i, j}, \alpha\right)}{(\alpha, \alpha)} & =2 n+2-i-j, \\
\Lambda_{i, j+n_{\mid h^{-}}} & =\frac{n-i+j}{2}\left(\tilde{\varepsilon}_{1}-\tilde{\varepsilon}_{2}\right)+2 \sum_{k=3}^{i+2} \tilde{\varepsilon}_{k}+\sum_{k=i+3}^{n+2-j} \tilde{\varepsilon}_{k}, \\
\frac{2\left(\nu_{i, j+n}, \alpha\right)}{(\alpha, \alpha)} & =n+1-i-j \quad\left(\alpha=\tilde{\varepsilon}_{1}+\tilde{\varepsilon}_{2}\right) .
\end{aligned}
$$

Comments. In Proposition 17.10, we prove that $\pi_{n-1}$ is an irreducible component of $\pi_{n-1, n+1}, \pi_{n-2, n+2}$ and of $\pi_{n-2, n+1}$ only. Also the $P^{i, j+n}$ 's with $i+j+n=2 n$ are the ones for which the real root $\alpha$ is simple. All this together with the same remarks as for $\operatorname{SU}(n, 1)$, explain the content of the table for all the discrete series representations, except $\pi_{n}$. The result for $\pi_{n}$ follows from Theorem 17.16.

\section{TABLE IV. $F_{4}$}

Let $\pi_{i}, 1 \leqslant i \leqslant 3$, be the discrete series representation associated with $\delta_{F_{i}}$ (cf. 11.10).

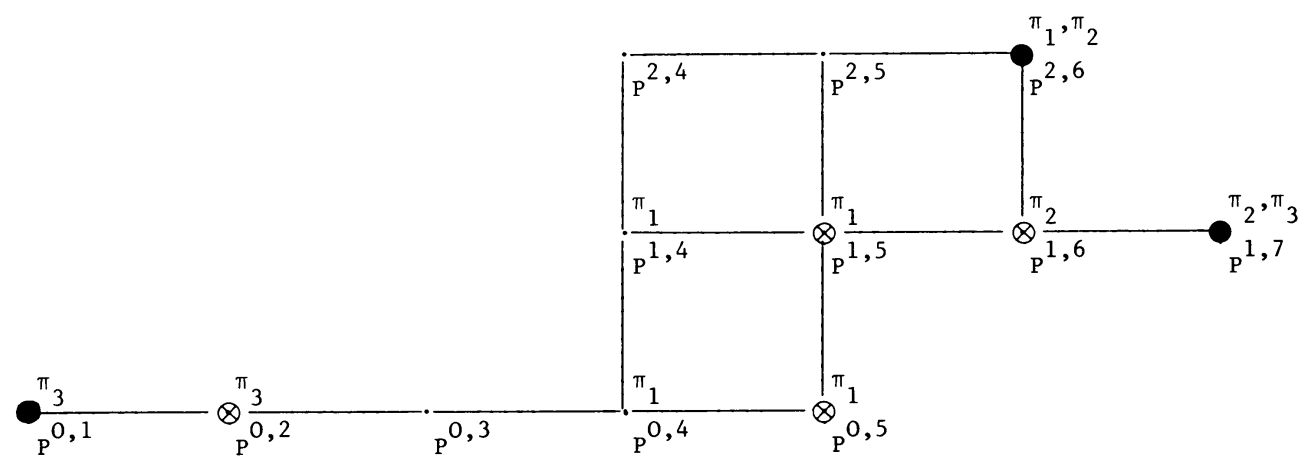

The parameters for the principal series $\pi_{\Lambda_{i, j \mid b^{-}, \nu_{i, j}}}$ associated with the $P^{i, j}$ 's are in order $\left(P^{0,1}, \ldots, P^{0,5}, P^{1,4}, \ldots, P^{1,7}, P^{2,4}, \ldots, P^{\Lambda_{i, j},-, \nu_{i, j}}\right)$ :

$$
\begin{gathered}
\Lambda_{i, j b_{1}-}:(0,0,0),\left(\frac{1}{2}, \frac{1}{2}, \frac{1}{2}\right),(1,1,0),(2,1,0),(2,2,0),\left(\frac{5}{2}, \frac{1}{2}, \frac{1}{2}\right),\left(\frac{5}{2}, \frac{3}{2}, \frac{1}{2}\right), \\
\left(\frac{5}{2}, \frac{3}{2}, \frac{3}{2}\right),(2,2,2),(3,0,0),(3,1,0),(3,1,1)
\end{gathered}
$$

and

$$
\nu_{i, j}: 11,10,9,7,5,6,4,2,1,5,3,1 \text {. }
$$

Comments. Since $\alpha=\tilde{\varepsilon}_{1}$ is simple for $P^{1,7}$ and $P^{2,5}$, this explains $\bullet$ Lemma 17.6 says that $\pi_{3}$ appears in $\pi_{0,1}$ and is a subspace. Hence the situation for $\pi_{3}$ is clear by Corollary 9.3.

For $\pi_{2}$ the result follows by Corollary 17.8.

Let $\tau_{\mu}$ be the irreducible $K$-type of highest weight $\mu=4 \varepsilon_{1}+4 \varepsilon_{2}+2 \varepsilon_{3} \cdot \tau_{\mu}$ is a $K$-type of $\pi_{1}$ with multiplicity one. The proof, using Blattner's multiplicity formula is straightforward, but deals with many different possibilities, hence we omit it. On 
the other hand using 17.2, it can be easily seen that $\tau_{\mu}$ is a $K$-type of $\pi_{1}$ of multiplicity greater than or equal to one. Since $\tau_{\mu}$ is a $K$-type only of the following principal series: $\pi_{0,5}(m=1), \pi_{1,5}(m=2), \pi_{1,6}(m=2), \pi_{1,7}(m=1), \pi_{2,5}(m=1)$ and $\pi_{2,6}(m=3)$, ( $m$ being the multiplicity of $\tau_{\mu}$ in the corresponding principal series), we are forced to conclude that $\pi_{1}$ does not appear in $\pi_{1,4}$ and $\pi_{0,4}$. Therefore $\pi_{1}$ can only be an irreducible component of $\pi_{1,5}$ and $\pi_{0,5}$ besides $\pi_{2,6}$ (Lemma 15.9).

By Theorem 4.15, Corollary 4.18 and Proposition 4.19 of [25], the composition series of $\pi_{1,5}$ and $\pi_{0,5}$ "look like" those of certain principal series for $\operatorname{Sp}(2,1)$. In particular, Table III then implies that $\pi_{1}$ does occur in both $\pi_{1,5}$ and $\pi_{0,5}$.

\section{REFERENCES}

1. W. Baldoni Silva, Branching theorems for semisimple Lie groups of real rank one, Rend. Sem. Univ. Padova (to appear).

2. N. Bourbaki, Groupes et algèbres de Lie, Hermann, Paris, 1968, Chapitres 4, 5 and 6.

3. Harish-Chandra, Discrete series for semisimple Lie groups. I, Acta Math. 113 (1965), 241-318.

4. __ Discrete series for semisimple Lie groups. II, Acta Math. 116 (1966), 1-111.

5. On some applications of the universal enveloping algebra of a semisimple Lie algebra, Trans. Amer. Math. Soc. 70 (1951), 28-96.

6. H. Hecht and W. Schmid, A proof of Blattner's conjecture, Invent. Math. 31 (1975), 129-154.

7. S. Helgason, Differential geometry and symmetry spaces, Academic Press, New York, 1962.

8. P. I. Holod and A. U. Klimyk, Representation of the group $\operatorname{Sp}(n, 1)$. I, Acad. Sci. Ukrain. SSR, Institute for Theoretical Physics, 1977 (preprint).

9. K. Johnson and N. Wallach, Composition series and intertwining operators for the spherical principal series, Bull. Amer. Math. Soc. 78 (1972), 1053-1059.

10. A. Knapp and N. Wallach, Szegö kernels associated with discrete series, Invent. Math. 311 (1976), $163-200$.

11. A. Knapp and G. Zuckerman, Classification of irreducible tempered representations of semisimple Lie groups, Proc. Nat. Acad. Sci. U.S.A. 73 (1976), 2178-2180.

12. R. P. Langlands, On the classification of irreducible representations of real algebraic groups, Inst. for Advanced Study, Princeton, N. J., 1973 (mimeographed notes).

13. J. Lepowsky, Ph. D. thesis, M.I.T.

14. H. Midorikawa, On a relation between characters of discrete and nonunitary principal series representations, Proc. Japan Acad. 50 (1974), 29-32.

15. W. Schmid, Some properties of square integrable representations of semisimple Lie groups, Ann. of Math. (2) 102 (1975), 535-564.

16. D. Vogan, The algebraic structure of the representations of semisimple Lie groups, 1977 (preprint).

17. N. Wallach, Harmonic analysis on homogeneous spaces, Dekker, New York, 1973.

18. Cyclic vectors and irreducibility for principal series representations. II, Trans. Amer. Math. Soc. 164 (1962), 389-396.

19. On the Enright-Varadarajan modules: construction of the discrete series, Ann. Ecole Norm. Sup. 9 (1976), 81-102.

20. G. Warner, Harmonic analysis on semisimple Lie groups, Springer-Verlag, Berlin, 1972.

21. G. Zuckerman, Some character identities for semisimple Lie groups, Thesis, Princeton Univ., Princeton, N. J., 1975.

22. , Tensor products of finite and infinite dimensional representations of semisimple Lie groups, 1977 (preprint).

23. N. Jacobson, Lie algebras, Interscience, New York, 1962.

24. H. Kraljevic, Representations of the universal covering group of the group $\operatorname{SU}(n, 1)$, Glasnik Mat. Ser. III 8 (28) (1973), 23-73.

25. B. Speh and D. Vogan, Reducibility of generalized principal series representations (preprint).

26. E. Thieleker, On the quasi-simple representations of the Lorentz group, Trans. Amer. Math. Soc. 179 (1973), 465-505.

Dipartimento di Matematica e Fisica, Libera Università Degli Studi di Trento, 38050-Povo (TRENTO), Italy 